\title{
Strong almost reducibility for analytic and Gevrey quasi-periodic cocycles
}

\author{
Claire Chavaudret \\ Institut de Mathématiques de Jussieu \\ 175 rue du Chevaleret, 75013 Paris, France
}

\begin{abstract}
This paper is about almost reducibility of quasi-periodic cocycles with a diophantine frequency which are sufficiently close to a constant. Generalizing previous works by L.H.Eliasson, we show a strong version of almost reducibility for analytic and Gevrey cocycles, that is to say, almost reducibility where the change of variables is in an analytic or Gevrey class which is independent of how close to a constant the initial cocycle is conjugated. This implies a result of density, or quasi-density, of reducible cocycles near a constant. Some algebraic structure can also be preserved, by doubling the period if needed.
\end{abstract}

\section{Introduction}

We are concerned with quasi-periodic cocycles, that is, solutions of equations of the form

$$
\forall(\theta, t) \in 2 \mathbb{T}^{d} \times \mathbb{R}, \frac{d}{d t} X^{t}(\theta)=A(\theta+t \omega) X^{t}(\theta) ; X^{0}(\theta)=I d
$$

where $A \in C^{0}\left(2 \mathbb{T}^{d}, \mathcal{G}\right)$ and $\mathcal{G}$ is a linear Lie algebra. Here $\mathbb{T}^{d}=\mathbb{R}^{d} / \mathbb{Z}^{d}$ stands for the $d$-torus, $d \geq 1$, and $2 \mathbb{T}^{d}=\mathbb{R}^{d} /\left(2 \mathbb{Z}^{d}\right)$ stands for the double torus. We will assume in this article that $\omega \in \mathbb{R}^{d}$ satisfies some diophantine conditions. The solution of (1) is called the quasi-periodic cocycle associated to $A$ and is defined on $2 \mathbb{T}^{d} \times \mathbb{R}$ with values in the connected component of the identity of a Lie group $G$ whose associated Lie algebra is $\mathcal{G}$. Terminology is explained by the fact that $A$ is the envelope of a quasi-periodic function, since $t \mapsto A(\theta+t \omega)$ is a quasi-periodic function for all $\theta \in 2 \mathbb{T}^{d}$. We say $X$ is a constant cocycle if $A$ is constant. A constant cocycle is always of the form $t \mapsto e^{t A}$.

A cocycle is said to be reducible if it is conjugated to a constant cocycle, in a sense that will be defined later on. The problem of reducibility of cocycles has been thoroughly studied and is of interest because the dynamics of reducible cocycles is well understood and because this problem has links with the spectral theory of Schrödinger cocycles and with the problem of lower dimensional invariant tori in hamiltonian systems. In the periodic case $(d=1)$, Floquet theory tells that every cocycle is reducible modulo a loss of periodicity. However, the problem is far more difficult if $d$ is greater than 1 and it is not true that every cocycle is then reducible. The question becomes whether every cocycle 
is close, up to a conjugacy, to a reducible one; from this question comes the notion of almost-reducibility. A cocycle is said to be almost-reducible, roughly speaking, if it can be conjugated to a cocycle which is arbitrarily close to a reducible one. Reductibility implies almost reducibility, however the reverse is not true: there are non reducible cocycles even close to a constant cocycle (see [4]). Almost reducibility is an interesting notion since the dynamics of an almost reducible cocycle are quite well known on a very long time.

We first focus on cocycles generated by functions which are analytic on a neighbourhood of the torus, i.e real analytic functions which are periodic in the direction of the real axis (recall that they are matrix-valued). For such a function $F$, we will let

$$
|F|_{r}=\sup _{|\operatorname{Im} \theta| \leq r}\|F(\theta)\|
$$

where $\|$.$\| stands for the operator norm.$

The aim of this paper is to show that for

$$
G=G L(n, \mathbb{C}), G L(n, \mathbb{R}), S L(2, \mathbb{C}), S L(n, \mathbb{R}), S p(n, \mathbb{R}), O(n), U(n)
$$

in the neighbourhood of a constant cocycle, every cocycle which is analytic on an $r$ neighbourhood of the torus and $G$-valued is almost reducible in $C_{r^{\prime}}^{\omega}\left(2 \mathbb{T}^{d}, G\right)$ for all $0<r^{\prime}<r \leq \frac{1}{2}$. The width of the neighbourhood only depends on the dimensions $n, d$, on the diophantine class of $\omega$, on the constant cocycle and on the loss of analyticity $r-r^{\prime}$.

More precisely, we shall prove the following theorem, for $G$ among the groups cited above and $\mathcal{G}$ the Lie algebra associated to $G$ :

Theorem 1.1 Let $0<r^{\prime}<r \leq \frac{1}{2}, A \in \mathcal{G}, F \in C_{r}^{\omega}\left(\mathbb{T}^{d}, \mathcal{G}\right)$. There is $\epsilon_{0}<1$ depending only on $n, d, \omega, A, r-r^{\prime}$ such that if

$$
|F|_{r} \leq \epsilon_{0}
$$

then for all $\epsilon>0$, there exists $\bar{A}_{\epsilon}, \bar{F}_{\epsilon} \in C_{r^{\prime}}^{\omega}\left(2 \mathbb{T}^{d}, \mathcal{G}\right), \Psi_{\epsilon}, Z_{\epsilon} \in C_{r^{\prime}}^{\omega}\left(2 \mathbb{T}^{d}, G\right)$ and $A_{\epsilon} \in \mathcal{G}$ such that for all $\theta \in 2 \mathbb{T}^{d}$,

$$
\partial_{\omega} Z_{\epsilon}(\theta)=(A+F(\theta)) Z_{\epsilon}(\theta)-Z_{\epsilon}(\theta)\left(\bar{A}_{\epsilon}(\theta)+\bar{F}_{\epsilon}(\theta)\right)
$$

with

1. $\partial_{\omega} \Psi_{\epsilon}=\bar{A}_{\epsilon} \Psi_{\epsilon}-\Psi_{\epsilon} A_{\epsilon}$,

2. $\left|\bar{F}_{\epsilon}\right|_{r^{\prime}} \leq \epsilon$,

3. $\left|\Psi_{\epsilon}\right|_{r^{\prime}} \leq \epsilon^{-\frac{1}{8}}$,

4. and $\left|Z_{\epsilon}-I d\right|_{r^{\prime}} \leq 2 \epsilon_{0}^{\frac{1}{2}}$.

Moreover, in dimension 2 or if $G=G L(n, \mathbb{C})$ or $U(n), Z_{\epsilon}, \bar{A}_{\epsilon}, \bar{F}_{\epsilon}$ are continuous on $\mathbb{T}^{d}$. 
Property 1 states the reducibility of $\bar{A}_{\epsilon}$. Theorem 1.1 immediately entails the following:

Theorem 1.2 Let $0<r^{\prime}<r \leq \frac{1}{2}, A \in \mathcal{G}, F \in C_{r}^{\omega}\left(\mathbb{T}^{d}, \mathcal{G}\right)$. There is $\epsilon_{0}<1$ depending only on $n, d, \omega, A, r-r^{\prime}$ such that if

$$
|F|_{r} \leq \epsilon_{0}
$$

then for all $\epsilon>0$, there exists $F_{\epsilon} \in C_{r^{\prime}}^{\omega}\left(2 \mathbb{T}^{d}, \mathcal{G}\right), Z_{\epsilon} \in C_{r^{\prime}}^{\omega}\left(2 \mathbb{T}^{d}, G\right)$ and $A_{\epsilon} \in \mathcal{G}$ such that for all $\theta \in 2 \mathbb{T}^{d}$,

$$
\partial_{\omega} Z_{\epsilon}(\theta)=(A+F(\theta)) Z_{\epsilon}(\theta)-Z_{\epsilon}(\theta)\left(A_{\epsilon}+F_{\epsilon}(\theta)\right)
$$

with $\left|F_{\epsilon}\right|_{r^{\prime}} \leq \epsilon$.

Note that in Theorem 1.2, we do not have any good estimate of $Z_{\epsilon}$. Theorem 1.1 also holds if one chooses $F$ in a class which is bigger than $C_{r}^{\omega}\left(\mathbb{T}^{d}, \mathcal{G}\right)$, i.e the class of functions in $C_{r}^{\omega}\left(2 \mathbb{T}^{d}, \mathcal{G}\right)$ satisfying some "nice periodicity properties" with respect to the matrix $A$.

There is a loss of analyticity in this result, but it is arbitrarily small. A result close to Theorem 1.1 in the case when $G=G L(n, \mathbb{R})$ had already been proven in [3] by L.H.Eliasson:

Let $A \in \operatorname{gl}(n, \mathbb{R})$ and $F \in C_{r}^{\omega}\left(\mathbb{T}^{d}, g l(n, \mathbb{R})\right)$. There is $\epsilon_{0}<1$ depending only on $n, d, \kappa, \tau,\|A\|, r$ such that if $|F|_{r} \leq \epsilon_{0}$, then for all $\epsilon>0$, there exists $0<r_{\epsilon}<r$, $Z_{\epsilon} \in C_{r_{\epsilon}}^{\omega}\left(2 \mathbb{T}^{d}, G L(n, \mathbb{R})\right)$ such that for all $\theta \in 2 \mathbb{T}^{d}$,

$$
\partial_{\omega} Z_{\epsilon}(\theta)=(A+F(\theta)) Z_{\epsilon}(\theta)-Z_{\epsilon}(\theta)\left(A_{\epsilon}+F_{\epsilon}(\theta)\right)
$$

with $A_{\epsilon} \in \operatorname{gl}(n, \mathbb{R}), F_{\epsilon} \in C_{r_{\epsilon}}^{\omega}\left(2 \mathbb{T}^{d}, g l(n, \mathbb{R})\right)$ and $\left|F_{\epsilon}\right|_{r_{\epsilon}} \leq \epsilon$.

Eliasson's theorem merely states almost reducibility in $C_{0}^{\omega}\left(2 \mathbb{T}^{d}, G L(n, \mathbb{R})\right)$, since the sequence $\left(r_{\epsilon}\right)$ might well tend to 0 . The achievement of Theorem 1.1 is to state almost reducibility in a more general algebraic framework, but also, and mostly, to show that almost reducibility holds in a fixed neighbourhood of a torus even when this torus has dimension greater than 1 . This is almost reducibility in a strong sense.

Note that, as was the case in [3], one cannot avoid to lose periodicity in theorem 1.1] if $G$ is a real group with dimension greater than 2. The notion of "nice periodicity properties" that will be given aims at limiting this loss to a period doubling. In comparison with the real framework, the symplectic framework does not introduce any new constraints in the elimination of resonances; therefore there is no more loss of periodicity here than in the case when $G=G L(n, \mathbb{R})$. As before in [2], a single period doubling is sufficient in the case when $G$ is a real symplectic group.

The second part of this paper is dedicated to showing that the same method gives an analogous result for cocycles which are in a Gevrey class (Theorem 3.1).

In dimension 2 or if $\mathcal{G}$ is $g l(n, \mathbb{C})$ or $u(n)$, these results can be rephrased as density of reducible cocycles in the neighbourhood of constant cocycles: 
Theorem 1.3 Let $\mathcal{G}=\operatorname{gl}(n, \mathbb{C}), u(n), g l(2, \mathbb{R}), \operatorname{sl}(2, \mathbb{R})$ or $o(2)$. Let $0<r^{\prime}<r \leq \frac{1}{2}$ and $A \in \mathcal{G}, F \in C_{r}^{\omega}\left(\mathbb{T}^{d}, \mathcal{G}\right)$. There is $\epsilon_{0}$ depending only on $r-r^{\prime}, n, d, \omega, A$ such that if

$$
|F|_{r} \leq \epsilon_{0}
$$

then for all $\epsilon>0$ there exists $H \in C_{r^{\prime}}^{\omega}\left(\mathbb{T}^{d}, \mathcal{G}\right)$ which is reducible in $C_{r^{\prime}}^{\omega}\left(\mathbb{T}^{d}, \mathcal{G}\right)$ and such that

$$
|A+F-H|_{r^{\prime}} \leq \epsilon
$$

A similar result, for smooth cocycles with values in compact Lie groups, was obtained by R. Krikorian in [7] (th.5.1.1). For cocycles over a rotation on the circle, analyticity is far better controlled (see for instance [1]) since it is then possible to use global methods. In this article, we are considering the case of a torus of arbitrary dimension. The KAM-type method that is being used here had already given way to full-measure reducibility results for cocycles with values in $S L(2, \mathbb{R})([4],[6])$.

\section{Sketch of the proof and organization of the paper}

The proof of Theorems 1.1 and 1.3 is a refinement of the method in [3; it is based on a KAM scheme. The central idea is to prove an inductive lemma where one conjugates a system which is close to a reducible one to another system which is even closer to something reducible. Iterating this lemma arbitrarily many times, one would then be able to conjugate the initial system to something which is arbitrarily close to a reducible one. An estimate on the reducing transformation would then imply almost reducibility. Now consider a system close to a reducible one; if it is close to a system which can be reduced to a constant part satisfying some non-resonant conditions, then there exists a conjugation which is close to the identity in a good topology taking the first system to something closer to a reducible system. But the constant part might well be too resonant for such a conjugation to exist. In this case, it is possible to remove the resonances in the constant part, but then the conjugation will not stay very close to the identity except if one accepts to give up a lot of regularity. Now we want to avoid this loss of regularity in order to obtain a strong version of almost reducibility. So we will have to improve the step of removing the resonances and use the following two facts: when resonances have been removed up to some order $N$, firstly, the eigenvalues will be so close together that resonances are in fact removed up to an order $R N$ which is much greater than $N$; secondly, the eigenvalues are removed in a durable way, that is, one will not have to remove resonances again until a great number of conjugations is made that will take the cocycle to something much closer to a reducible one. The article is organized as follows:

Section 2 is dedicated to the proof of the theorem in the analytic case. Here are the main steps of the proof:

- Removing of the resonances by a map $\Phi$ called a reduction of the eigenvalues at order $R, \bar{N}$ (Proposition 2.6) for $R, N \in \mathbb{N} \backslash\{0\}$. 
In dimension 2, $\Phi$ will be such that for all $H$ continuous on $\mathbb{T}^{d}, \Phi H \Phi^{-1}$ is continuous on $\mathbb{T}^{d}$.

This step is crucial in the obtention of strong almost reducibility. The reduction of the eigenvalues is defined in a way similar to [3], however here it will remove resonances up to an order $R \bar{N}$ which is much greater than the value of the parameter $\bar{N}$ appearing in the estimates. The parameter $R$ will be used to define a map of reduction of the eigenvalues at order $R, \bar{N}$ where $\bar{N}$ does not depend on the loss of analyticity. This way, the map of reduction of the eigenvalues will stay under control on a neighbourhood of the torus which will not have to fade totally.

- Resolution of the homological equation (Proposition 2.8): if $\tilde{A}$ has a spectrum fulfilling some non-resonance conditions and $\tilde{F}$ is a function with nice periodicity properties with respect to $\tilde{A}$, then there exists a solution $\tilde{X}$ of equation

$$
\partial_{\omega} \tilde{X}=[\tilde{A}, \tilde{X}]+\tilde{F}^{R \bar{N}} ; \hat{\tilde{X}}(0)=0
$$

having the same periodicity properties as $\tilde{F}$; it takes its values in the same Lie algebra as does $\tilde{F}$. Moreover, it can be well controlled by losing some analyticity.

- Inductive lemma (Proposition 2.14): If $\tilde{F} \in C_{r}^{\omega}\left(2 \mathbb{T}^{d}, \mathcal{G}\right)$ has some periodicity properties (with respect to $\tilde{A}$ ), if

$$
\partial_{\omega} \Psi=\bar{A} \Psi-\Psi \tilde{A}
$$

and $\bar{F}=\Psi \tilde{F} \Psi^{-1}$, then there exists $Z \in C_{r^{\prime}}^{\omega}\left(2 \mathbb{T}^{d}, G\right)$ such that

$$
\partial_{\omega} Z=(\bar{A}+\bar{F}) Z-Z\left(\bar{A}^{\prime}+\bar{F}^{\prime}\right)
$$

with $\bar{A}^{\prime}$ reducible, $\bar{F}^{\prime}$ is much smaller than $\bar{F}, Z$ is close to the identity and $\Psi^{\prime-1} \bar{F}^{\prime} \Psi^{\prime}$ has periodicity properties with respect to $A^{\prime}$ which are similar to the properties of $\tilde{F}$.

The estimate of $\bar{F}^{\prime}$ depends on $\tilde{F}-\tilde{F}^{R \bar{N}}$, on the reduction of the eigenvalues $\Phi$, and on the solution $\tilde{X}$ of the homological equation.

- Iteration of the inductive lemma (Theorem [2.16): We shall iterate Lemma 2.14 so as to obtain estimates of analytic functions on a sequence of neighbourhoods of the torus not tending to 0, by means of a numerical lemma (Lemma 2.15), to reduce the perturbation arbitrarily.

In section 3, some lemmas are given (3.1) which show that it is possible to adapt the proof to the Gevrey case; namely, the estimates will be analogous to those that are obtained in the analytic case and so, by slightly modifying the parameters, the argument works in the same way: one obtains analogous reduction of the eigenvalues (3.2), homological equation (3.3) and inductive lemmas (3.4). 


\section{Notations, further definitions and a general assumption}

For a function $f \in C^{1}\left(2 \mathbb{T}^{d}, g l(n, \mathbb{C})\right)$, for all $\theta \in 2 \mathbb{T}^{d}$ we will denote by

$$
\partial_{\omega} f(\theta)=\frac{d}{d t} f(\theta+t \omega)_{\mid t=0}
$$

the derivative of $f$ in the direction $\omega$. Denote by $\langle.,$.$\rangle the complex euclidian scalar$ product, taking it antilinear in the second variable. For a linear operator $M$, we shall call $M^{*}$ its adjoint, which is identical to the transpose of $M$ if $M$ is real. Also denote by $M_{\mathcal{N}}$ the nilpotent part of $M$, as follows: let $M=P A P^{-1}$ with $A$ in Jordan normal form, let $A_{D}$ be the diagonal part of $A$, then $M_{\mathcal{N}}=P\left(A-A_{D}\right) P^{-1}$. To simplify the writing, if $A: 2 \mathbb{T}^{d} \rightarrow G L(n, \mathbb{C})$, we will denote by $A^{-1}$ the map $\theta \mapsto A(\theta)^{-1}$. For all $m=\left(m_{1}, \ldots, m_{d}\right) \in \frac{1}{2} \mathbb{Z}^{d}$, we shall denote $|m|=\left|m_{1}\right|+\cdots+\left|m_{d}\right|$. The letter $J$ will stand for matrix $J=\left(\begin{array}{cc}0 & -I d \\ I d & 0\end{array}\right)$.

Definition: A function $f$ is analytic on an r-neighbourhood of the torus (resp. double torus) if $f$ is holomorphic on $\left\{x=\left(x_{1}, \ldots, x_{d}\right) \in \mathbb{C}^{d}, \sup _{j}\left|\operatorname{Im} x_{j}\right|<r\right\}$ and 1-periodic (resp. 2-periodic) in $\operatorname{Re} x_{j}$ for all $1 \leq j \leq d$.

For all subset $E$ of $g l(n, \mathbb{C})$, denote by $C_{r}^{\omega}\left(\mathbb{T}^{d}, E\right)$ the set of functions which are analytic on an $r$-neighbourhood of the torus and whose restriction to $\mathbb{R}^{d}$ takes its values in $E$; let $C_{r}^{\omega}\left(2 \mathbb{T}^{d}, E\right)$ be the set of functions which are analytic on an $r$-neighbourhood of the double torus and whose restriction to $2 \mathbb{T}^{d}$ takes its values in $E$. For all $f \in C_{r}^{\omega}\left(2 \mathbb{T}^{d}, E\right)$, denote

$$
|f|_{r}=\sup _{|\operatorname{Im} x|<r}\|f(x)\|
$$

where ||.|| stands for the operator norm.

Let $C_{r}^{G, \beta}$ be the class of Gevrey $\beta$ functions with parameter $r$, i.e functions $f$ satisfying

$$
\sum_{\alpha \in \mathbb{N}^{d}} \frac{r^{\beta|\alpha|}}{\alpha !^{\beta}} \sup _{\theta}\left\|\partial^{\alpha} F(\theta)\right\|<+\infty
$$

Denote by $\|.\|_{\beta, r}$ the norm

$$
\|F\|_{\beta, r}=\sum_{\alpha \in \mathbb{N}^{d}} \frac{r^{\beta|\alpha|}}{\alpha !^{\beta}} \sup _{\theta}\left\|\partial^{\alpha} F(\theta)\right\|
$$

To formalize the notion of reducibility, we shall introduce an equivalence relation on cocycles.

Definition: Let $G$ be a Lie group and $\mathcal{G}$ the Lie algebra associated to $G$. Let $r, r^{\prime}>0$ and $A, B \in C_{r}^{\omega}\left(2 \mathbb{T}^{d}, \mathcal{G}\right)$. We say that $A$ and $B$ are conjugate in $C_{r^{\prime}}^{\omega}\left(2 \mathbb{T}^{d}, G\right)$ if there exists $Z \in C_{r^{\prime}}^{\omega}\left(2 \mathbb{T}^{d}, G\right)$ such that for all $\theta \in 2 \mathbb{T}^{d}$,

$$
\partial_{\omega} Z(\theta)=A(\theta) Z(\theta)-Z(\theta) B(\theta)
$$


where $\partial_{\omega}$ means the derivative in the direction $\omega$. If $B$ is constant in $\theta$, we say that $A$ is reducible in $C_{r^{\prime}}^{\omega}\left(2 \mathbb{T}^{d}, G\right)$, or reducible by $Z$ to $B$. We will use an analogous definition with $C^{G, \beta}$ instead of $C^{\omega}$.

Note that if $X$ is the quasi-periodic cocycle associated to $A$, then the map $A$ is reducible by $Z$ to $B$ if and only if

$$
\forall(t, \theta), X^{t}(\theta)=Z(\theta+t \omega)^{-1} e^{t B} Z(\theta)
$$

Reducibility is also equivalent to the fact that the map from $2 \mathbb{T}^{d} \times \mathbb{R}^{n}$ to itself:

$$
\left(\begin{array}{c}
\theta \\
v
\end{array}\right) \mapsto\left(\begin{array}{c}
\theta+\omega \\
X^{1}(\theta) v
\end{array}\right)
$$

is conjugate to a map $\chi$ such that

$$
\frac{d \chi}{d \theta}\left(\begin{array}{c}
\theta \\
v
\end{array}\right) \equiv\left(\begin{array}{l}
\overline{1} \\
0
\end{array}\right)
$$

Assumption: The frequency $\omega$ is in the diophantine class $D C(\kappa, \tau)$, i.e

$$
\forall m \in \mathbb{Z}^{d} \backslash\{0\},|\langle m, \omega\rangle| \geq \frac{\kappa}{|m|^{\tau}}
$$

where $\kappa, \tau$ are fixed throughout the paper and $0<\kappa<1, \tau \geq \max (1, d-1)$.

\section{Strong almost reducibility for analytic quasi-periodic cocycles}

\subsection{Nice periodicity properties}

A few definitions will first be given. The notion of "triviality with respect to a decomposition" will make the construction of the map of reduction of the eigenvalues easier; the "nice periodicity properties" have been introduced in [3] and are used in the real case to make sure that only one period doubling will be needed in iterating the inductive lemma.

\subsubsection{Invariant decompositions}

The set $\mathcal{L}=\left\{L_{1}, \ldots, L_{R}\right\}$ is called a decomposition of $\mathbb{C}^{n}$ if $\mathbb{C}^{n}=\bigoplus_{j} L_{j}$. If $\mathcal{L}, \mathcal{L}^{\prime}$ are decompositions of $\mathbb{C}^{n}$, then $\mathcal{L}$ is said to be finer than $\mathcal{L}^{\prime}$ if for all $L \in \mathcal{L}$, there is $L^{\prime} \in \mathcal{L}^{\prime}$ such that $L \subset L^{\prime} ; \mathcal{L}$ is said strictly finer than $\mathcal{L}^{\prime}$ if $\mathcal{L}$ is finer than $\mathcal{L}^{\prime}$ and $\mathcal{L} \neq \mathcal{L}^{\prime}$.

Definition: Let $A \in g l(n, \mathbb{C})$; then $\mathcal{L}=\left\{L_{1}, \ldots, L_{s}\right\}$ is an $A$-decomposition, or else $A$-invariant decomposition, if it is a decomposition of $\mathbb{C}^{n}$ and for all $i, A L_{i} \subset L_{i}$. Subsets $L_{i}$ are called subspaces of $\mathcal{L}$.

Let a Jordan decomposition for $A$ be an $A$-decomposition which is minimal (i.e no finer decomposition is an $A$-decomposition). Then 
- A matrix might have many Jordan decompositions. For instance, the identity has infinitely many Jordan decompositions.

- A decomposition is an $A$-decomposition if and only if it is less fine than some Jordan decomposition for $A$. Therefore, if operators $A$ and $A^{\prime}$ have a common Jordan decomposition, then an $A$-decomposition which is less fine than this common Jordan decomposition is an $A^{\prime}$-decomposition.

Notation: Let $\mathcal{L}$ be an $A$-decomposition. For all $L \in \mathcal{L}$, denote by $\sigma\left(A_{\mid L}\right)$ the spectrum of the restriction of $A$ to subspace $L$.

Definition: Let $\kappa^{\prime} \geq 0$. Let $\mathcal{L}_{A, \kappa^{\prime}}$ be the unique $A$-decomposition $\mathcal{L}$ such that for all $L \neq L^{\prime} \in \mathcal{L}, \alpha \in \sigma\left(A_{\mid L}\right)$ and $\beta \in \sigma\left(A_{\mid L^{\prime}}\right) \Rightarrow|\alpha-\beta|>\kappa^{\prime}$ and such that no $A$-decomposition strictly finer than $\mathcal{L}$ has this property.

Remark: For $\kappa^{\prime} \geq 0$, any Jordan decomposition is finer than $\mathcal{L}_{A, \kappa^{\prime}}$.

Definition: Let $\mathcal{L}$ be a decomposition of $\mathbb{C}^{n}$. For all $u \in \mathbb{C}^{n}$, there is a unique decomposition $u=\sum_{L \in \mathcal{L}} u_{L}$ such that $u_{L} \in L$ for all $L \in \mathcal{L}$. For all $L \in \mathcal{L}$, the projection on $L$ with respect to $\mathcal{L}$, denoted by $P_{L}^{\mathcal{L}}$, is the map defined by $P_{L}^{\mathcal{L}} u=u_{L}$.

Remark: Let $A \in g l(n, \mathbb{C})$ and $\kappa^{\prime}>0$. If $\mathcal{L}$ is an $A$-decomposition which is less fine than $\mathcal{L}_{A, \kappa^{\prime}}$, then one has the following lemma, which can be found in [3], appendix, Lemma $A^{1}$ :

Lemma 2.1 There is a constant $C_{0} \geq 1$ depending only on $n$ such that for all subspace $L \in \mathcal{L}$,

$$
\left\|P_{L}^{\mathcal{L}}\right\| \leq C_{0}\left(\frac{1+\left\|A_{\mathcal{N}}\right\|}{\kappa^{\prime}}\right)^{n(n+1)}
$$

In what follows, $C_{0}$ will always stand for this constant fixed in Lemma 2.1.

Definition: An $\left(A, \kappa^{\prime}, \gamma\right)$-decomposition is an $A$-decomposition $\mathcal{L}$ such that for all $L \in \mathcal{L}$, the projection on $L$ with respect to $\mathcal{L}$ satisfies

$$
\left\|P_{L}^{\mathcal{L}}\right\| \leq C_{0}\left(\frac{1+\left\|A_{\mathcal{N}}\right\|}{\kappa^{\prime}}\right)^{\gamma}
$$

Remark: For $A \in \operatorname{gl}(n, \mathbb{C})$, one always has $A=\sum_{L, L^{\prime} \in \mathcal{L}} P_{L}^{\mathcal{L}} A P_{L^{\prime}}^{\mathcal{L}}$ In particular, if $\mathcal{L}$ is an $A$-decomposition, then $A=\sum_{L \in \mathcal{L}} P_{L}^{\mathcal{L}} A P_{L}^{\mathcal{L}}$.

Definitions: Let $\mathcal{L}$ be a decomposition. We say that

- $\mathcal{L}$ is a real decomposition if for all $L \in \mathcal{L}, \bar{L} \in \mathcal{L}$;

\footnotetext{
${ }^{1}$ Lemma A from [3] gives in fact an estimate which depends on $\|A\|$, but the proof shows clearly that the estimate in fact only depends on $A_{\mathcal{N}}$.
} 
- $\mathcal{L}$ is a symplectic decomposition if it is a decomposition of $\mathbb{C}^{n}$ with even $n$ and for all $L \in \mathcal{L}$, there is a unique $L^{\prime} \in \mathcal{L}$ such that $\left\langle L, J L^{\prime}\right\rangle \neq 0$;

- $\mathcal{L}$ is a unitary decomposition if for all $L \neq L^{\prime} \in \mathcal{L},\left\langle L, L^{\prime}\right\rangle=0$.

\section{Remark:}

- If $A$ is a real matrix, then for all $\kappa^{\prime} \geq 0, \mathcal{L}_{A, \kappa^{\prime}}$ is a real decomposition.

- For all $L$, there is at least one $L^{\prime}$ such that $\left\langle L, J L^{\prime}\right\rangle \neq 0$. This comes from the fact that the symplectic form $\langle., J$.$\rangle is non-degenerate.$

- If $A \in \operatorname{sp}(n, \mathbb{R})$, then any $A$-decomposition $\mathcal{L}$ which is less fine than $\mathcal{L}_{A, 0}$ is a real and symplectic decomposition. To see this, let $L, L^{\prime} \in \mathcal{L}$ such that $\left\langle L, J L^{\prime}\right\rangle \neq 0$; let $v \in L, v^{\prime} \in L^{\prime}$ be eigenvectors of $A$ such that $\left\langle v, J v^{\prime}\right\rangle \neq 0$ and $\lambda, \lambda^{\prime}$ their associated eigenvalues. Then

$$
\lambda\left\langle v, J v^{\prime}\right\rangle=\left\langle A v, J v^{\prime}\right\rangle=\left\langle v, A^{*} J v^{\prime}\right\rangle=-\left\langle v, J A v^{\prime}\right\rangle=-\bar{\lambda}^{\prime}\left\langle v, J v^{\prime}\right\rangle
$$

and since $\left\langle v, J v^{\prime}\right\rangle \neq 0$, then $\lambda=-\bar{\lambda}^{\prime}$.

- If $A \in U(n)$, then any decomposition which is less fine than $\mathcal{L}_{A, 0}$ is unitary.

- If $\mathcal{L}$ is unitary, then for every $L \in \mathcal{L}, P_{L}^{\mathcal{L}}$ is an orthogonal projection so

$$
\left\|P_{L}^{\mathcal{L}}\right\| \leq 1
$$

\subsubsection{Triviality and nice periodicity properties with respect to a decomposi- tion}

Definition: Let $\mathcal{L}$ be a decomposition of $\mathbb{C}^{n}$. We say a map $\Psi$ is trivial with respect to $\mathcal{L}$ if there exist $\left\{m_{L}, L \in \mathcal{L}\right\} \subset \frac{1}{2} \mathbb{Z}^{d}$ such that for all $\theta \in 2 \mathbb{T}^{d}$,

$$
\Psi(\theta)=\sum_{L \in \mathcal{L}} e^{2 i \pi\left\langle m_{L}, \theta\right\rangle} P_{L}^{\mathcal{L}}
$$

We say that the function $\Psi$ is trivial if there exists a decomposition $\mathcal{L}$ such that $\Psi$ is trivial with respect to $\mathcal{L}$.

\section{Remark:}

- If $\Psi$ is trivial with respect to $\mathcal{L}$ and $\mathcal{L}^{\prime}$ is finer than $\mathcal{L}$, then $\Psi$ is trivial with respect to $\mathcal{L}^{\prime}$.

- If $\Phi, \Psi: 2 \mathbb{T}^{d} \rightarrow G L(n, \mathbb{C})$ are trivial with respect to $\mathcal{L}$, then the product $\Phi \Psi$ is trivial with respect to $\mathcal{L}$.

- If $\Phi$ is trivial with respect to an $A$-decomposition $\mathcal{L}$, then for all $\theta \in 2 \mathbb{T}^{d},[A, \Phi(\theta)]=$ 0 . 
Lemma 2.2 Let $\mathcal{L}$ be a real decomposition of $\mathbb{C}^{n},\left\{m_{L}, L \in \mathcal{L}\right\} \subset \frac{1}{2} \mathbb{Z}^{d}$ and $\Psi$ defined by

$$
\Psi(\theta)=\sum_{L \in \mathcal{L}} e^{2 i \pi\left\langle m_{L}, \theta\right\rangle} P_{L}^{\mathcal{L}}
$$

Then $\Psi$ is real if and only if for all $L, m_{L}=-m_{\bar{L}}$. Moreover, if $\Psi$ is real, then $\Psi$ takes its values in $S L(n, \mathbb{R})$.

Proof: Assume that for all $L \in \mathcal{L}, m_{L}=-m_{\bar{L}}$. Let $u \in \mathbb{R}^{n}$. Then

$$
\overline{\Psi(\theta) u}=\sum_{L \in \mathcal{L}} e^{2 i \pi\left\langle-m_{L}, \theta\right\rangle} \overline{P_{L}^{\mathcal{L}} u}=\sum_{L \in \mathcal{L}} e^{2 i \pi\left\langle m_{\bar{L}}, \theta\right\rangle} P_{\bar{L}}^{\mathcal{L}} u=\Psi(\theta) u
$$

so $\Psi(\theta)$ is real.

Now suppose that $\Psi$ is real. Then for all $\theta$,

$$
\sum_{L \in \mathcal{L}} e^{2 i \pi\left\langle m_{L}, \theta\right\rangle} P_{L}^{\mathcal{L}}=\sum_{L \in \mathcal{L}} e^{2 i \pi\left\langle-m_{L}, \theta\right\rangle} \overline{P_{L}^{\mathcal{L}}}=\sum_{L \in \mathcal{L}} e^{2 i \pi\left\langle-m_{L}, \theta\right\rangle} P_{\bar{L}}^{\mathcal{L}}
$$

so $m_{L}=-m_{\bar{L}}$.

Suppose $\Psi$ is real; then for all $L, m_{L}=-m_{\bar{L}}$ so $\Psi(\theta)$ is the exponential of a trace-zero matrix, so it has determinant 1 .

Remark: Any map which is trivial with respect to a unitary decomposition is unitary: let $\mathcal{L}$ be a unitary decomposition, let $\Phi$ be trivial with respect to $\mathcal{L}$ and let $L, L^{\prime} \in \mathcal{L}$. Then for all $u \in \mathcal{L}, v \in \mathcal{L}^{\prime}$,

$$
\langle\Phi(\theta) u, \Phi(\theta) v\rangle=\left\langle e^{2 i \pi\left\langle m_{L}, \theta\right\rangle} u, e^{2 i \pi\left\langle m_{L^{\prime}}, \theta\right\rangle} v\right\rangle=\langle u, v\rangle
$$

Lemma 2.3 Let $\mathcal{L}$ be a real and symplectic decomposition and $\left\{m_{L}, L \in \mathcal{L}\right\}$ be a family of elements of $\frac{1}{2} \mathbb{Z}^{d}$. Let $\Psi=\sum_{L \in \mathcal{L}} e^{2 i \pi\left\langle m_{L}, \cdot\right\rangle} P_{L}^{\mathcal{L}}$. Then $\Psi$ takes its values in $\operatorname{Sp}(n, \mathbb{R})$ if and only if

- for all $L, m_{L}=-m_{\bar{L}}$

- and if $\left\langle L, J L^{\prime}\right\rangle \neq 0$, then $m_{L}=m_{L^{\prime}}$.

Proof: By Lemma 2.2, $\Psi$ is real if and only if for all $L, m_{L}=-m_{\bar{L}}$. Assume now $\Psi$ is real.

We show first that if for all $L, L^{\prime} \in \mathcal{L},\left\langle L, J L^{\prime}\right\rangle \neq 0 \Rightarrow m_{L}=m_{L^{\prime}}$, then $\Psi$ takes its values in $S p(n, \mathbb{R})$. Let $u, v \in \mathbb{R}^{n}$. Then

$$
\left\langle u, \Psi(\theta)^{*} J \Psi(\theta) v\right\rangle=\langle\Psi(\theta) u, J \Psi(\theta) v\rangle=\sum_{L} e^{2 i \pi\left\langle m_{L}-m_{M(L)}, \theta\right\rangle}\left\langle P_{L}^{\mathcal{L}} u, J P_{M(L)}^{\mathcal{L}} v\right\rangle
$$

where $M(L)$ stands for the unique subspace such that $\langle L, J M(L)\rangle \neq 0$. Assume that if $\left\langle L, J L^{\prime}\right\rangle \neq 0$, then $m_{L}=m_{L^{\prime}}$. This implies that 


$$
\left\langle u, \Psi(\theta)^{*} J \Psi(\theta) v\right\rangle=\sum_{L}\left\langle P_{L}^{\mathcal{L}} u, J P_{M(L)}^{\mathcal{L}} v\right\rangle=\langle u, J v\rangle
$$

so $\Psi(\theta) \in S p(n, \mathbb{R})$.

Now we will show that if $\Psi(\theta) \in S p(n, \mathbb{R})$ and if $\left\langle L, J L^{\prime}\right\rangle \neq 0$, then $m_{L}=m_{L^{\prime}}$. Suppose $\Psi(\theta) \in S p(n, \mathbb{R})$. For any two vectors $u, v$,

$$
\langle u, J v\rangle=\left\langle u, \Psi(\theta)^{*} J \Psi(\theta) v\right\rangle=\langle\Psi(\theta) u, J \Psi(\theta) v\rangle
$$

If $u \in L$ and $v \in m(L)$ satisfy $\langle u, J v\rangle \neq 0$, then

$$
\langle u, J v\rangle=\langle\Psi(\theta) u, J \Psi(\theta) v\rangle=e^{2 i \pi\left\langle m_{L}-m_{M(L)}, \theta\right\rangle}\langle u, J v\rangle
$$

so $m_{L}=m_{M(L)}$.

We will now define the periodicity properties.

Definition: Let $\mathcal{L}$ be a decomposition of $\mathbb{C}^{n}$. We say that $F \in C^{0}\left(2 \mathbb{T}^{d}, g l(n, \mathbb{R})\right)$ has nice periodicity properties with respect to $\mathcal{L}$ if there exists a map $\Phi$ which is trivial with respect to $\mathcal{L}$ and such that $\Phi^{-1} F \Phi$ is continuous on $\mathbb{T}^{d}$.

To make the family $\left(m_{L}\right)$ explicit, we say that $F$ has nice periodicity properties with respect to $\mathcal{L}$ and $\left(m_{L}\right)$.

\section{Remark:}

- If $F \in C^{0}\left(2 \mathbb{T}^{d}, g l(n, \mathbb{R})\right)$ has nice periodicity properties with respect to a decomposition $\mathcal{L}$ and $\Phi$ is trivial with respect to $\mathcal{L}$, then $\Phi F \Phi^{-1}$ has nice periodicity properties with respect to $\mathcal{L}$.

- If $\mathcal{L}^{\prime}$ is a decomposition of $\mathbb{C}^{n}$ which is finer than $\mathcal{L}$ and $F$ has nice periodicity properties with respect to $\mathcal{L}$, then $F$ has nice periodicity properties with respect to $\mathcal{L}^{\prime}$.

- Let $\mathcal{L}$ be a decomposition of $\mathbb{C}^{n}$ and $\left(m_{L}\right)_{L \in \mathcal{L}}$ be a family of elements of $\frac{1}{2} \mathbb{Z}^{d}$. If $F_{1}, F_{2} \in C^{0}\left(2 \mathbb{T}^{d}, g l(n, \mathbb{R})\right)$ have nice periodicity properties with respect to $\mathcal{L}$ and $\left(m_{L}\right)$, then the product $F_{1} F_{2}$ has nice periodicity properties with respect to $\mathcal{L}$ and $\left(m_{L}\right)$.

\subsection{Removing the resonances}

In the following we will have to solve a homological equation and estimate the solution on a neighbourhood of the torus; in order to have a sufficient estimate, one will assume that the coefficients of the equation satisfy some diophantine conditions:

Let $A \in \operatorname{gl}(n, \mathbb{R})$ and $0<\kappa^{\prime}<1$. Let $N \in \mathbb{N}$.

Definition: Let $z \in \mathbb{C}, \nu \in\{1,2\}$. We say that $z$ is diophantine modulo $\nu$ with respect to $\omega$, with constant $\kappa^{\prime}$, exponent $\tau$ and order $N$ if for every $m \in \frac{1}{\nu} \mathbb{Z}^{d}$ such that $0<|m| \leq N$, 


$$
|z-2 i \pi\langle m, \omega\rangle| \geq \frac{\kappa^{\prime}}{|m|^{\tau}}
$$

This property will be denoted by

$$
z \in D C_{\omega, \nu}^{N}\left(\kappa^{\prime}, \tau\right)
$$

Note that

$$
D C_{\omega, 2}^{N}\left(\kappa^{\prime}, \tau\right) \subset D C_{\omega, 1}^{N}\left(\kappa^{\prime}, \tau\right)
$$

and that every real number $z$ is in $D C_{\omega, 2}^{N}\left(\frac{\kappa}{2^{\tau}}, \tau\right)$ since for all $m \in \frac{1}{2} \mathbb{Z}^{d}$,

$$
|z-2 i \pi\langle m, \omega\rangle|=\left(|z|^{2}+(2 \pi|\langle m, \omega\rangle|)^{2}\right)^{\frac{1}{2}} \geq \frac{\pi \kappa}{|2 m|^{\tau}} \geq \frac{\kappa}{|2 m|^{\tau}}
$$

Remark: In the definition above, the condition is required only for non vanishing $m$, so (13) has a meaning.

Definition: $A$ is said to have $D C_{\omega}^{N}\left(\kappa^{\prime}, \tau\right)$ spectrum if

$$
\left\{\begin{array}{c}
\forall \alpha, \beta \in \sigma(A), \alpha-\beta \in D C_{\omega, 1}^{N}\left(\kappa^{\prime}, \tau\right) \\
\forall \alpha, \beta \in \sigma(A), \alpha \neq \bar{\beta} \Rightarrow \alpha-\beta \in D C_{\omega, 2}^{N}\left(\kappa^{\prime}, \tau\right)
\end{array}\right.
$$

Let $N \in \mathbb{N}$. Let $A$ in a Lie algebra $\mathcal{G}$. The aim is to show that there exists $\kappa^{\prime}>0, \tilde{A} \in \mathcal{G}$ such that $\tilde{A}$ has $D C_{\omega}^{N}\left(\kappa^{\prime}, \tau\right)$ spectrum and $A$ and $\tilde{A}$ are conjugate (in the acception of cocycles, following the definition given in the introduction). To achieve this, one has to find a family $\left(m_{1}, \ldots, m_{n}\right)$ satisfying

$$
\left\{\begin{array}{c}
\forall \alpha_{j}, \alpha_{k} \in \sigma(A), \alpha_{j}-\alpha_{k}+2 i \pi\left\langle m_{j}-m_{k}, \omega\right\rangle \in D C_{\omega, 1}^{N}\left(\kappa^{\prime}, \tau\right) \\
\forall \alpha_{j}, \alpha_{k} \in \sigma(A), \alpha_{j} \neq \bar{\alpha}_{k} \Rightarrow \alpha_{j}-\alpha_{k}+2 i \pi\left\langle m_{j}-m_{k}, \omega\right\rangle \in D C_{\omega, 2}^{N}\left(\kappa^{\prime}, \tau\right)
\end{array}\right.
$$

We shall construct the so-called map of reduction of the eigenvalues $\Phi$ conjugating (in the sense of cocycles) $A$ to the matrix obtained from $A$ by substituting an eigenvalue $\alpha_{j}$ by $\alpha_{j}+2 i \pi\left\langle m_{j}, \omega\right\rangle$, then we will prove that $\Phi$ is $G$-valued.

\subsubsection{Diophantine conditions}

Lemma 2.4 Let $\left\{\alpha_{1}, \ldots, \alpha_{n}\right\} \subset \mathbb{C}$. Let $\tilde{N} \in \mathbb{N}$ and $\kappa^{\prime} \leq \frac{\kappa}{n(8 \tilde{N})^{\tau}}$. There exists $m_{1}, \ldots, m_{n} \in$ $\frac{1}{2} \mathbb{Z}^{d}$ such that $\sup _{j}\left|m_{j}\right| \leq \tilde{N}$, and such that letting for all $j, \tilde{\alpha}_{j}=\alpha_{j}-2 i \pi\left\langle m_{j}, \omega\right\rangle$, then

$$
\begin{gathered}
\left\{\alpha_{1}, \ldots, \alpha_{n}\right\}=\overline{\left\{\alpha_{1}, \ldots, \alpha_{n}\right\}} \Rightarrow \forall j, k, \alpha_{j}=\bar{\alpha}_{k} \Rightarrow m_{j}=-m_{k} \\
n=2, \alpha_{2}=-\alpha_{1} \Rightarrow m_{1}=-m_{2} \\
\forall j, k, \alpha_{j}=-\bar{\alpha}_{k} \Rightarrow m_{j}=m_{k}
\end{gathered}
$$




$$
\begin{gathered}
\forall j, k,\left|\alpha_{j}-\alpha_{k}\right| \leq \kappa^{\prime} \Rightarrow m_{j}=m_{k} \\
\forall j,\left|I m \tilde{\alpha}_{j}\right| \leq\left|I m \alpha_{j}\right| \\
\forall j, k, \alpha_{j}=\bar{\alpha}_{k} \Rightarrow \tilde{\alpha}_{j}-\tilde{\alpha}_{k} \in D C_{\omega, 1}^{\tilde{N}}\left(\kappa^{\prime}, \tau\right)
\end{gathered}
$$

and

$$
\forall j, k, \alpha_{j} \neq \bar{\alpha}_{k} \Rightarrow \tilde{\alpha}_{j}-\tilde{\alpha}_{k} \in D C_{\omega, 2}^{\tilde{N}}\left(\kappa^{\prime}, \tau\right)
$$

and such that if not all $m_{j}$ vanish, then there exist $j, k$ such that

$$
\left|\alpha_{j}-\alpha_{k}\right| \geq \kappa^{\prime},\left|\tilde{\alpha}_{j}-\tilde{\alpha}_{k}\right|<\kappa^{\prime}
$$

Moreover, there exist $m_{1}, \ldots m_{n} \in \mathbb{Z}^{d}$, with $\left|m_{j}\right| \leq \tilde{N}$ for all $j$, fulfilling conditions (21), (22), (23), such that

$$
\forall j, k, \quad \tilde{\alpha}_{j}-\tilde{\alpha}_{k} \in D C_{\omega, 1}^{\tilde{N}}\left(\kappa^{\prime}, \tau\right)
$$

and such that if not all $m_{j}$ vanish, then there exist $j, k$ such that (26) holds.

Proof: We shall proceed in two steps. The first step consists in removing resonances which might occur between two eigenvalues whose imaginary parts are nearly opposite to each other. Once this first lot of resonances is removed, the second step consists in removing the resonances which might occur between two eigenvalues whose imaginary parts are far from opposite.

- Let $1 \leq j \leq n$. Suppose that there is an $m \in \mathbb{Z}^{d}, 0<|m| \leq \tilde{N}$ such that

$$
\left|2 \operatorname{Im} \alpha_{j}-2 \pi\langle m, \omega\rangle\right|<\frac{\kappa^{\prime}}{|m|^{\tau}}
$$

then let $\alpha_{j}^{\prime}=\alpha_{j}-2 i \pi\left\langle\frac{m}{2}, \omega\right\rangle$. Otherwise, let $\alpha_{j}^{\prime}=\alpha_{j}$. Note that if $\left|\alpha_{j}-\alpha_{k}\right| \leq \kappa^{\prime}$ and if there exist $m_{j} \neq m_{k}$ such that

$$
\left|2 \operatorname{Im} \alpha_{j}-2 \pi\left\langle m_{j}, \omega\right\rangle\right|<\frac{\kappa^{\prime}}{\left|m_{j}\right|^{\tau}} ;\left|2 \operatorname{Im} \alpha_{k}-2 \pi\left\langle m_{k}, \omega\right\rangle\right|<\frac{\kappa^{\prime}}{\left|m_{k}\right|^{\tau}}
$$

then

$$
\left|2 i \pi\left\langle m_{j}-m_{k}, \omega\right\rangle\right| \leq \frac{\kappa}{\left|m_{j}-m_{k}\right|^{\tau}}
$$

which is impossible since $\omega$ is diophantine. Therefore conditions (19) to (24) hold with $\alpha_{j}^{\prime}=\tilde{\alpha}_{j}$ and $m_{j}$ such that $\alpha_{j}-\alpha_{j}^{\prime}=2 i \pi\left\langle m_{j}, \omega\right\rangle$.

- Let $I_{-r}, \ldots, I_{r}$ be the finest partition of $\{1, \ldots, n\}$ such that 


$$
\left|\operatorname{Im}\left(\alpha_{j}^{\prime}-\alpha_{k}^{\prime}\right)\right| \leq \kappa^{\prime} \Rightarrow \exists-r \leq r^{\prime} \leq r \mid j, k \in I_{r^{\prime}}
$$

and choose the indices in such a way that

$$
r^{\prime}<r^{\prime \prime} \Rightarrow \forall j \in I_{r^{\prime}}, \forall k \in I_{r^{\prime \prime}}, I m \alpha_{j}^{\prime} \leq \operatorname{Im} \alpha_{k}^{\prime}
$$

Note that $I_{0}$ might be empty. We will proceed by induction on $r^{\prime}$ to prove the following property $\mathcal{P}\left(r^{\prime}\right)$ :

There are $m_{1}^{\prime}, m_{-1}^{\prime}, \ldots, m_{r^{\prime}}^{\prime}, m_{-r^{\prime}}^{\prime} \in \mathbb{Z}^{d}$ with $\sup _{|j| \leq r^{\prime}}\left|m_{j}^{\prime}\right| \leq \tilde{N}$ such that properties (19) to (25) hold for all $-r^{\prime} \leq r_{1}, r_{2} \leq r^{\prime}, j \in I_{r_{1}}, k \in I_{r_{2}}$ with $m_{j}^{\prime}$ instead of $m_{j}$ and $\alpha_{j}^{\prime}$ instead of $\alpha_{j}$.

- Case $r^{\prime}=0$ : if $I_{0}$ is empty, then $\mathcal{P}(0)$ trivially holds. Assume $I_{0}$ is non empty. Then for all $j, k \in I_{0}$ and all $m \in \frac{1}{2} \mathbb{Z}^{d}$ such that $0<|m| \leq \tilde{N}$,

$$
\left|\alpha_{j}^{\prime}-\alpha_{k}^{\prime}-2 i \pi\langle m, \omega\rangle\right| \geq\left|\operatorname{Im}\left(\alpha_{j}^{\prime}-\alpha_{k}^{\prime}\right)-2 \pi\langle m, \omega\rangle\right| \geq \frac{\kappa}{|m|^{\tau}}-n \kappa^{\prime} \geq \kappa^{\prime}
$$

so $\alpha_{j}^{\prime}-\alpha_{k}^{\prime} \in D C_{\omega, 2}^{\tilde{N}}\left(\kappa^{\prime}, \tau\right)$ and $\mathcal{P}(0)$ holds true.

- Let $r^{\prime} \leq r-1$. Assume $\mathcal{P}\left(r^{\prime}\right)$ holds. Consider $I_{r^{\prime}+1}$ and $I_{-r^{\prime}-1}$. There are two possible cases.

- There exist $-r^{\prime} \leq r^{\prime \prime} \leq r^{\prime}, j \in I_{r^{\prime \prime}}, k \in I_{r^{\prime}+1}$ and $m \in \mathbb{Z}^{d}$ such that $|m| \leq \tilde{N}$ and

$$
\left|\alpha_{j}^{\prime}-\alpha_{k}^{\prime}-2 i \pi\left\langle m_{r^{\prime \prime}}+m, \omega\right\rangle\right|<\frac{\kappa^{\prime}}{|m|^{\tau}}
$$

- The case above does not hold.

In the first case, let $m_{r^{\prime}+1}^{\prime}=m=-m_{-r^{\prime}-1}^{\prime}$. In the second case, let $m_{r^{\prime}+1}^{\prime}=m_{-r^{\prime}-1}^{\prime}=0$.

Now $m_{r^{\prime}+1}^{\prime}$ and $m_{-r^{\prime}-1}^{\prime}$ are independent from $j, k$. To see this, suppose there are $j_{1}, j_{2} \in$ $I_{r_{1}}, k_{1}, k_{2} \in I_{r_{2}}, m_{1} \neq m_{2} \in \mathbb{Z}^{d}$ such that for $l=1,2$,

$$
\left|\alpha_{j_{l}}^{\prime}-\alpha_{k_{l}}^{\prime}-2 i \pi\left\langle m_{l}, \omega\right\rangle\right|<\frac{\kappa^{\prime}}{\left|m_{l}\right|^{\tau}}
$$

Then

$$
\left|2 \pi\left\langle m_{1}-m_{2}, \omega\right\rangle\right| \leq \frac{\kappa}{\left|m_{1}-m_{2}\right|^{\tau}}
$$

which is impossible. Therefore $\mathcal{P}\left(r^{\prime}+1\right)$ holds true.

- Once $m_{1}^{\prime}, \ldots, m_{r}^{\prime}, m_{-1}^{\prime}, \ldots, m_{-r}^{\prime} \in \mathbb{Z}^{d}$ are defined, conditions (19) to (25) hold with, for all $j \in I_{r^{\prime}}, \tilde{\alpha}_{j}=\alpha_{j}^{\prime}-2 i \pi\left\langle m_{r^{\prime}}^{\prime}, \omega\right\rangle$ and $m_{j}$ such that $\alpha_{j}-\tilde{\alpha}_{j}=2 i \pi\left\langle m_{j}, \omega\right\rangle$. Condition (26) is obvious by construction. 
- By proceeding only with the second step, one gets $m_{1}, \ldots m_{n} \in \mathbb{Z}^{d}$, with $\left|m_{j}\right| \leq \tilde{N}$ for all $j$, satisfying conditions (21), (22), (23), such that

$$
\forall j, k, \tilde{\alpha}_{j}-\tilde{\alpha}_{k} \in D C_{\omega, 1}^{\tilde{N}}\left(\kappa^{\prime}, \tau\right)
$$

and such that if not all $m_{j}$ vanish, then there are $j, k$ such that (26) holds true.

Lemma 2.5 Let $\left\{\alpha_{1}, \ldots, \alpha_{n}\right\} \subset \mathbb{C}$. For every $R, N \in \mathbb{N}, N \geq 2, R \geq 1$, there exists $\bar{N} \in\left[N, R^{\frac{1}{2} n(n-1)} N\right]$ and $m_{1}, \ldots, m_{n} \in \frac{1}{2} \mathbb{Z}^{d}$ with

$$
\sup _{j}\left|m_{j}\right| \leq 2 \bar{N}
$$

such that letting $\tilde{\alpha}_{j}=\alpha_{j}-2 i \pi\left\langle m_{j}, \omega\right\rangle$ and

$$
\kappa^{\prime \prime}=\frac{\kappa}{n\left(8 R^{\frac{1}{2} n(n-1)+1} N\right)^{\tau}}
$$

conditions (19) to (23) of Lemma 2.4 hold for $\kappa^{\prime}=\kappa^{\prime \prime}$, and such that

$$
\forall j, k, \quad \tilde{\alpha}_{j}-\tilde{\alpha}_{k} \in D C_{\omega, 1}^{R \bar{N}}\left(\kappa^{\prime \prime}, \tau\right)
$$

and

$$
\forall j, k, \alpha_{j} \neq \bar{\alpha}_{k} \Rightarrow \tilde{\alpha}_{j}-\tilde{\alpha}_{k} \in D C_{\omega, 2}^{R \bar{N}}\left(\kappa^{\prime \prime}, \tau\right)
$$

Moreover, there exist $m_{1}, \ldots m_{n} \in \mathbb{Z}^{d}$ with $\left|m_{j}\right| \leq \bar{N}$ for all $j$ such that conditions (21), (22), (23) and (30) hold true.

Proof: If $\alpha_{j}$ satisfy for all $j, k$

$$
\left\{\begin{array}{l}
\alpha_{j}=\bar{\alpha}_{k} \Rightarrow \alpha_{j}-\alpha_{k} \in D C_{\omega, 1}^{R N}\left(\kappa^{\prime \prime}, \tau\right) \\
\alpha_{j} \neq \bar{\alpha}_{k} \Rightarrow \alpha_{j}-\alpha_{k} \in D C_{\omega, 2}^{R N}\left(\kappa^{\prime \prime}, \tau\right)
\end{array}\right.
$$

then we are done with $\bar{N}=N$ and $m_{1}=\cdots=m_{n}=0$.

Suppose (32) does not hold. Then apply Lemma 2.4 with $\tilde{N}=R N, \kappa^{\prime}=\kappa^{\prime \prime}$ to get $m_{1}^{1}, \ldots, m_{n}^{1}$ such that

$$
\left\{\begin{array}{c}
\forall j, k, \alpha_{j}=\bar{\alpha}_{k} \Rightarrow m_{j}^{1}=-m_{k}^{1} \\
\forall j, k, \alpha_{j}=-\bar{\alpha}_{k} \Rightarrow m_{j}^{1}=m_{k}^{1} \\
\forall j, k,\left|\alpha_{j}-\alpha_{k}\right| \leq \kappa^{\prime \prime} \Rightarrow m_{j}^{1}=m_{k}^{1} \\
\forall j,\left|I m \alpha_{j}-2 i \pi\left\langle m_{j}^{1}, \omega\right\rangle\right| \leq\left|I m \alpha_{j}\right|
\end{array}\right.
$$

and

$$
\left\{\begin{array}{l}
\alpha_{j}=\bar{\alpha}_{k} \Rightarrow \alpha_{j}-\alpha_{k}-2 i \pi\left\langle m_{j}^{1}-m_{k}^{1}, \omega\right\rangle \in D C_{\omega, 1}^{R N}\left(\kappa^{\prime \prime}, \tau\right) \\
\alpha_{j} \neq \bar{\alpha}_{k} \Rightarrow \alpha_{j}-\alpha_{k}-2 i \pi\left\langle m_{j}^{1}-m_{k}^{1}, \omega\right\rangle \in D C_{\omega, 2}^{R N}\left(\kappa^{\prime \prime}, \tau\right)
\end{array}\right.
$$

and such that there exist $j_{1}, k_{1}$ satisfying $\left|\operatorname{Im}\left(\alpha_{j_{1}}-\alpha_{k_{1}}\right)-2 i \pi\left\langle m_{j_{1}}^{1}-m_{k_{1}}^{1}, \omega\right\rangle\right|<\kappa^{\prime \prime}$.

Assume there are $m_{1}^{r}, \ldots, m_{n}^{r}$ such that $\sup \left|m_{j}^{r}\right| \leq\left(R+R^{2}+\cdots+R^{r}\right) N$ and that for all $j, k$, 


$$
\left\{\begin{array}{c}
\forall j, k, \alpha_{j}=\bar{\alpha}_{k} \Rightarrow m_{j}^{r}=-m_{k}^{r} \\
\forall j, k, \alpha_{j}=-\bar{\alpha}_{k} \Rightarrow m_{j}^{r}=m_{k}^{r} \\
\forall j, k,\left|\alpha_{j}-\alpha_{k}\right| \leq \kappa^{\prime \prime} \Rightarrow m_{j}^{r}=m_{k}^{r} \\
\forall j,\left|I m \alpha_{j}-2 i \pi\left\langle m_{j}^{r}, \omega\right\rangle\right| \leq\left|I m \alpha_{j}\right|
\end{array}\right.
$$

and

$$
\left\{\begin{array}{l}
\alpha_{j}=\bar{\alpha}_{k} \Rightarrow \alpha_{j}-\alpha_{k}-2 i \pi\left\langle m_{j}^{r}-m_{k}^{r}, \omega\right\rangle \in D C_{\omega, 1}^{R^{r} N}\left(\kappa^{\prime \prime}, \tau\right) \\
\alpha_{j} \neq \bar{\alpha}_{k} \Rightarrow \alpha_{j}-\alpha_{k}-2 i \pi\left\langle m_{j}^{r}-m_{k}^{r}, \omega\right\rangle \in D C_{\omega, 2}^{R^{r} N}\left(\kappa^{\prime \prime}, \tau\right)
\end{array}\right.
$$

and suppose there exist distinct $\left(j_{1}, k_{1}\right), \ldots,\left(j_{r}, k_{r}\right)$ such that for all $l \leq r$,

$$
\left|I m \alpha_{j_{l}}-I m \alpha_{k_{l}}-2 i \pi\left\langle m_{j_{l}}^{r}-m_{k_{l}}^{r}, \omega\right\rangle\right|<\kappa^{\prime \prime}
$$

If moreover one has for all $j, k$

$$
\left\{\begin{array}{l}
\alpha_{j}=\bar{\alpha}_{k} \Rightarrow \alpha_{j}-\alpha_{k}-2 i \pi\left\langle m_{j}^{r}-m_{k}^{r}, \omega\right\rangle \in D C_{\omega, 1}^{R^{r+1} N}\left(\kappa^{\prime \prime}, \tau\right) \\
\alpha_{j} \neq \bar{\alpha}_{k} \Rightarrow \alpha_{j}-\alpha_{k}-2 i \pi\left\langle m_{j}^{r}-m_{k}^{r}, \omega\right\rangle \in D C_{\omega, 2}^{R^{r+1} N}\left(\kappa^{\prime \prime}, \tau\right)
\end{array}\right.
$$

then the process ends with $\bar{N}=R^{r} N$ and $m_{j}=m_{j}^{r}$ since it is true that

$$
\left|m_{j}^{r}\right| \leq\left(R+R^{2}+\cdots+R^{r}\right) N \leq R^{r} N \frac{1-\frac{1}{R^{r}}}{1-\frac{1}{R}} \leq 2 R^{r} N
$$

Otherwise, iterate once more Lemma 2.4 with $\tilde{N}=R^{r+1} N$ and $\alpha_{j}-2 i \pi\left\langle m_{j}^{r}, \omega\right\rangle$ in place of $\alpha_{j}$ to get $m_{1}^{r+1}, \ldots, m_{n}^{r+1}$ such that $\sup \left|m_{j}^{r+1}\right| \leq\left(R+R^{2}+\cdots+R^{r+1}\right) N$ and for all $j, k$,

$$
\left\{\begin{array}{c}
\forall j, k, \alpha_{j}=\bar{\alpha}_{k} \Rightarrow m_{j}^{r+1}=-m_{k}^{r+1} \\
\forall j, k, \alpha_{j}=-\bar{\alpha}_{k} \Rightarrow m_{j}^{r+1}=m_{k}^{r+1} \\
\forall j, k,\left|\alpha_{j}-\alpha_{k}\right| \leq \kappa^{\prime \prime} \Rightarrow m_{j}^{r+1}=m_{k}^{r+1} \\
\forall j,\left|I m \alpha_{j}-2 i \pi\left\langle m_{j}^{r+1}, \omega\right\rangle\right| \leq\left|I m \alpha_{j}\right|
\end{array}\right.
$$

and

$$
\left\{\begin{array}{l}
\alpha_{j}=\bar{\alpha}_{k} \Rightarrow \alpha_{j}-\alpha_{k}-2 i \pi\left\langle m_{j}^{r+1}-m_{k}^{r+1}, \omega\right\rangle \in D C_{\omega, 1}^{R^{r+1} N}\left(\kappa^{\prime \prime}, \tau\right) \\
\alpha_{j} \neq \bar{\alpha}_{k} \Rightarrow \alpha_{j}-\alpha_{k}-2 i \pi\left\langle m_{j}^{r+1}-m_{k}^{r+1}, \omega\right\rangle \in D C_{\omega, 2}^{R^{r+1} N}\left(\kappa^{\prime \prime}, \tau\right)
\end{array}\right.
$$

and that there exist distinct $\left(j_{1}, k_{1}\right), \ldots,\left(j_{r+1}, k_{r+1}\right)$ such that for all $l \leq r+1$,

$$
\left|I m \alpha_{j_{l}}-\operatorname{Im} \alpha_{k_{l}}-2 i \pi\left\langle m_{j_{l}}^{r+1}-m_{k_{l}}^{r+1}, \omega\right\rangle\right|<\kappa^{\prime \prime}
$$

Therefore, for all $1 \leq l \leq r+1$,

$$
\left|\alpha_{j_{l}}-\alpha_{k_{l}}-2 i \pi\left\langle m_{j_{l}}^{r+1}-m_{k_{l}}^{r+1}, \omega\right\rangle\right|<\kappa^{\prime \prime}
$$

This implies that for all $m \in \frac{1}{2} \mathbb{Z}^{d}$ such that $0<|m| \leq R \bar{N}$ and for all $l, 1 \leq l \leq r+1$,

$$
\left|\alpha_{j_{l}}-\alpha_{k_{l}}-2 i \pi\left\langle m_{j_{l}}^{r+1}-m_{k_{l}}^{r+1}, \omega\right\rangle-2 i \pi\langle m, \omega\rangle\right| \geq \frac{\kappa}{2^{\tau+1}(R \bar{N})^{\tau}}-\kappa^{\prime \prime} \geq \kappa^{\prime \prime}
$$


so for all $l \leq r+1$,

$$
\alpha_{j_{l}}-\alpha_{k_{l}}-2 i \pi\left\langle m_{j_{l}}^{r+1}-m_{k_{l}}^{r+1}, \omega\right\rangle \in D C_{\omega, 2}^{R \bar{N}}\left(\kappa^{\prime \prime}, \tau\right)
$$

Therefore, after $\bar{r} \leq \frac{n(n-1)}{2}$ steps, one gets conditions (30) and (31) with $m_{j}=m_{j}^{\bar{r}}$ and $\tilde{\alpha}_{j}=\alpha_{j}-2 i \pi\left\langle m_{j}, \omega\right\rangle$ and $\left|\operatorname{Im} \alpha_{j}-2 i \pi\left\langle m_{j}, \omega\right\rangle\right| \leq\left|\operatorname{Im} \alpha_{j}\right|$. It is true that $\left|m_{j}^{\bar{r}}\right| \leq 2 N$ and conditions (19) to (23) of Lemma 2.4 are also satisfied.

Lemma 2.4 implies that if conditions (19) and (31) are not required, then one can get $m_{1}, \ldots m_{n} \in \mathbb{Z}^{d}$.

\subsubsection{Reduction of the eigenvalue}

Now the preceding lemmas will be used to define the map of reduction of the eigenvalues $\Phi$ which will conjugate $A$ to a matrix with $D C_{\omega}^{R N}\left(\kappa^{\prime \prime}, \tau\right)$ spectrum for some $\kappa^{\prime \prime}$, with $R, N$ arbitrarily great and $\Phi$ bounded independently of $R$.

In all that follows, $G$ will be a Lie group among

$$
G L(n, \mathbb{C}), G L(n, \mathbb{R}), S p(n, \mathbb{R}), S L(2, \mathbb{C}), S L(n, \mathbb{R}), O(n), U(n)
$$

and $\mathcal{G}$ will be the Lie algebra associated to $G$.

Proposition 2.6 Let $A \in \mathcal{G}, R \geq 1$ and $N \in \mathbb{N}$. There exists $\bar{N} \in\left[N, R^{\frac{1}{2} n(n-1)} N\right]$ such that if

$$
\kappa^{\prime \prime}=\frac{\kappa}{n\left(8 R^{\frac{1}{2} n(n-1)+1} N\right)^{\tau}}
$$

then there exists a map $\Phi$ which is trivial with respect to $\mathcal{L}_{A, \kappa^{\prime \prime}}$ and $G$-valued and such that

1. for all $r^{\prime} \geq 0$,

$$
|\Phi|_{r^{\prime}} \leq n C_{0}\left(\frac{1+\left\|A_{\mathcal{N}}\right\|}{\kappa^{\prime \prime}}\right)^{n(n+1)} e^{4 \pi \bar{N} r^{\prime}},\left|\Phi^{-1}\right|_{r^{\prime}} \leq n C_{0}\left(\frac{1+\left\|A_{\mathcal{N}}\right\|}{\kappa^{\prime \prime}}\right)^{n(n+1)} e^{4 \pi \bar{N} r^{\prime}}
$$

2. Let $\tilde{A}$ be such that

$$
\forall \theta \in 2 \mathbb{T}^{d}, \partial_{\omega} \Phi(\theta)=A \Phi(\theta)-\Phi(\theta) \tilde{A}
$$

then

$$
\|\tilde{A}-A\| \leq 4 \pi \bar{N}
$$

and $\tilde{A}$ has $D C_{\omega}^{R \bar{N}}\left(\kappa^{\prime \prime}, \tau\right)$ spectrum. 
3. If $\mathcal{G}=\operatorname{gl}(n, \mathbb{C})$ or $u(n), \Phi$ is defined on $\mathbb{T}^{d}$.

4. If $\mathcal{G}=o(n)$ or $u(n)$, then

$$
|\Phi|_{r^{\prime}} \leq n e^{4 \pi \bar{N} r^{\prime}},\left|\Phi^{-1}\right|_{r^{\prime}} \leq n e^{4 \pi \bar{N} r^{\prime}}
$$

5. If $\mathcal{G}=\operatorname{sl}(2, \mathbb{C})$ or $\operatorname{sl}(2, \mathbb{R})$, then either $\Phi$ is the identity or $\|\tilde{A}\| \leq \kappa^{\prime \prime}$.

Proof: Let $\left\{\alpha_{1}, \ldots, \alpha_{n}\right\}=\sigma(A)$. Two cases must be considered:

- If $\mathcal{G}=g l(n, \mathbb{C})$ or $u(n)$, Lemma 2.5 gives $\bar{N}$ and $m_{j} \in \mathbb{Z}^{d}$ for $j=1, \ldots, n$ such that

$$
N \leq \bar{N} \leq R^{\frac{1}{2} n(n-1)} N ; \sup _{j}\left|m_{j}\right| \leq 2 \bar{N}
$$

and such that conditions (21) to (23) of Lemma 2.4 hold with $\kappa^{\prime}=\kappa^{\prime \prime}$, as well as conditions (30).

- If $\mathcal{G}=g l(n, \mathbb{R}), \operatorname{sp}(n, \mathbb{R}), \operatorname{sl}(n, \mathbb{R}), \operatorname{sl}(2, \mathbb{C})$ or $o(n)$, Lemma 2.5 gives $\bar{N}$ and $m_{j} \in \frac{1}{2} \mathbb{Z}^{d}$ for $j=1, \ldots, n$ such that

$$
N \leq \bar{N} \leq R^{\frac{1}{2} n(n-1)} N ; \sup _{j}\left|m_{j}\right| \leq 2 \bar{N}
$$

and such that conditions (19) to (23) of Lemma 2.4 hold with $\kappa^{\prime}=\kappa^{\prime \prime}$, as well as conditions (30) and (31).

For all $j$ there is a unique $L \in \mathcal{L}_{A, \kappa^{\prime \prime}}$ such that $\alpha_{j} \in \sigma\left(A_{\mid L}\right)$. Let $m_{L}=m_{j}$. Then $m_{L}$ is independent of $j$ thanks to property (22).

For all $\theta \in 2 \mathbb{T}^{d}$, let

$$
\Phi(\theta)=\sum_{L \in \mathcal{L}_{A, \kappa^{\prime \prime}}} e^{2 i \pi\left\langle m_{L}, \theta\right\rangle} P_{L}^{\mathcal{L}_{A, \kappa^{\prime \prime}}}
$$

By construction of the $\left(m_{L}\right), \Phi$ is defined on $\mathbb{T}^{d}$ if $\mathcal{G}=g l(n, \mathbb{C})$ or $u(n)$. Let us prove that $\Phi$ is $G$-valued.

- if $\mathcal{G}=\operatorname{gl}(n, \mathbb{C})$, this is trivial;

- if $\mathcal{G}=\operatorname{sl}(2, \mathbb{C})$ or $\operatorname{sl}(2, \mathbb{R})$, this comes from condition (20);

- if $\mathcal{G}=u(n), \Phi$ has unitary values.

- if $\mathcal{G}=g l(n, \mathbb{R})$, this comes from Lemma 2.2, since $\mathcal{L}_{A, \kappa^{\prime \prime}}$ is a real decomposition and according to Lemma 2.5 , for all $L \in \mathcal{L}_{A, \kappa^{\prime \prime}}, m_{L}=-m_{\bar{L}}$.

- if $\mathcal{G}=o(n)$, the map $\Phi$ has values in real unitary matrices, i.e orthogonal matrices. 
- if $\mathcal{G}=\operatorname{sp}(n, \mathbb{R}), \mathcal{L}_{A, \kappa^{\prime \prime}}$ is a symplectic decomposition. Lemma 2.5 ensures that for all $L \in \mathcal{L}_{A, \kappa^{\prime \prime}}, m_{L}=-m_{\bar{L}}$ and

$$
\forall L, L^{\prime} \in \mathcal{L}_{A, \kappa^{\prime \prime}},\left\langle L, J L^{\prime}\right\rangle \neq 0 \Rightarrow m_{L}=m_{L^{\prime}}
$$

Therefore Lemma 2.3 implies that for all $\theta$ the matrix $\Phi(\theta)$ is in $S p(n, \mathbb{R})$.

Properties (30) and (31) ensure that $\tilde{A}$ has $D C_{\omega}^{R \bar{N}}\left(\kappa^{\prime \prime}, \tau\right)$ spectrum.

Moreover, for all $L \in \overline{\mathcal{L}},\left|m_{L}\right| \leq 2 \bar{N}$. The estimate of each $P_{L}^{\overline{\mathcal{L}}}$ recalled in Lemma 2.1 implies that $\Phi$ satisfies the estimate

$$
|\Phi|_{r^{\prime}} \leq n C_{0}\left(\frac{1+\left\|A_{\mathcal{N}}\right\|}{\kappa^{\prime \prime}}\right)^{n(n+1)} e^{4 \pi \bar{N} r^{\prime}}
$$

and so does $\Phi^{-1}$ since

$$
\Phi^{-1}=\sum_{L \in \mathcal{L}_{A, \kappa^{\prime \prime}}} e^{-2 i \pi\left\langle m_{L}, \cdot\right\rangle} P_{L}^{\mathcal{L}_{A, \kappa^{\prime \prime}}}
$$

Now if $\mathcal{G}$ is $o(n)$ or $u(n)$, then every projection $P_{L}^{\mathcal{L}_{A, \kappa^{\prime \prime}}}$ has norm 1 and therefore $\Phi$ and $\Phi^{-1}$ satisfy (50). By definition of $\tilde{A}$,

$$
\forall L \in \mathcal{L}^{\prime}, \sigma\left(\tilde{A}_{\mid L}\right)=\sigma\left(A_{\mid L}\right)-2 i \pi\left\langle m_{L}, \omega\right\rangle
$$

and by property (23),

$$
\forall \alpha \in \sigma\left(A_{\mid L}\right),\left|\alpha-2 i \pi\left\langle m_{L}, \omega\right\rangle\right| \leq|\alpha|
$$

Let $P$ be such that $P A P^{-1}$ is in Jordan normal form, let $\alpha_{j}$ be the eigenvalues of $A$ and $p_{j}$ the columns of $P$, then for all $j$,

$$
\left\|(\tilde{A}-A) p_{j}\right\|=\left\|2 i \pi\left\langle m_{j}, \omega\right\rangle p_{j}\right\| \leq 4 \pi \bar{N}\left\|p_{j}\right\|
$$

So $\|\tilde{A}-A\| \leq 4 \pi \bar{N}$, whence property (49). Finally, if $\mathcal{G}=\operatorname{sl}(2, \mathbb{C})$ or $\operatorname{sl}(2, \mathbb{R})$, then either $\tilde{A}=A$, or $A$ is diagonalizable, and then $\tilde{A}$ is also diagonalizable, so their norms are the modulus of their eigenvalues and by condition (26) $),\|\tilde{A}\| \leq \kappa^{\prime \prime}$.

Definition: A map $\Phi$ satisfying the conclusion of Proposition 2.6 will be called a map of reduction of the eigenvalues of $A$ at order $R, \bar{N}$.

In dimension 2, the map of reduction of the eigenvalues $\Phi$ satisfies the following property: for every function $H$ continuous on $\mathbb{T}^{d}$ and with values in $g l(2, \mathbb{C}), \Phi H \Phi^{-1}$ and $\Phi^{-1} H \Phi$ are continuous on $\mathbb{T}^{d}$.

Dimension 2 has, indeed, the particularity that every decomposition $\mathcal{L}$ of $\mathbb{R}^{2}$ at most two subpaces $L_{1}, L_{2}$, in which case $m_{L_{1}}+m_{L_{2}} \in \mathbb{Z}^{d}$ (if the decomposition is trivial, $m_{L}=0$ ). In any case, $\sum_{L \in \mathcal{L}} m_{L} \in \mathbb{Z}^{d}$. 


\subsection{Homological equation}

Solving the homological equation is a first step towards reducing the perturbation.

Notation: For every function $F \in L^{2}\left(2 \mathbb{T}^{d}\right)$ and every $N \in \mathbb{N}$, we will denote by $F^{N}$ and call truncation of $F$ at order $N$ the function that one obtains by truncating the Fourier series of $F$ :

$$
F^{N}(\theta)=\sum_{|m| \leq N} \hat{F}(m) e^{2 i \pi\langle m, \theta\rangle}
$$

The following lemma will be useful in the solving of the homological equation.

Lemma 2.7 Let $f, g$ be trigonometric polynomial with $g$ real on $\mathbb{R}^{d}$. Let $\left.r>0, r^{\prime} \in\right] 0, r[$ and suppose that there exists $C$ such that $|f|_{r^{\prime}} \leq C|g|_{r}$. Then for all $m \in \frac{1}{2} \mathbb{Z}^{d}$,

$$
\left|f e^{2 i \pi\langle m, .\rangle}\right|_{r^{\prime}} \leq C\left|g e^{2 i \pi\langle m, .\rangle}\right|_{r}
$$

Proof: Since $g$ is real,

$$
\forall m \in \mathbb{Z}^{d}, \overline{\hat{g}(-m)}=\hat{g}(m)
$$

so for all $x$ and all $y \in[-r, r]^{d}$,

$g(x-i y)=\sum_{m} \hat{g}(m) e^{2 i \pi\langle m, x-i y\rangle}=\sum_{m} \overline{\hat{g}(-m)} e^{2 i \pi\langle-m,-x+i y\rangle}=\overline{\sum_{m} \hat{g}(-m) e^{2 i \pi\langle-m, x+i y\rangle}}=\overline{g(x+i y)}$

which implies that for all $x, y$,

$$
|g(x-i y)|=|g(x+i y)|
$$

Let us show that for every $m \in \mathbb{Z}^{d}$,

$$
|g|_{r} e^{2 \pi|m| r}=\left|g e^{2 i \pi\langle m, .\rangle}\right|_{r}
$$

By the maximum principle,

$$
|g|_{r}=\sup _{x ;\left|y_{j}\right| \leq r, 1 \leq j \leq d}|g(x+i y)|=\sup _{x ;\left|y_{j}\right|=r, 1 \leq j \leq d}|g(x+i y)|
$$

Let $y_{0}$ such that

$$
|g|_{r}=\sup _{x}\left|g\left(x+i y_{0}\right)\right|
$$

then, for $m$ having only one non-zero component $m_{j}$, either

$$
|g|_{r} e^{2 \pi|m| r}=\sup _{x}\left|g\left(x+i y_{0}\right)\right|\left|e^{2 i \pi\left\langle m, x+i y_{0}\right\rangle}\right|=\left|g e^{2 i \pi\langle m, .\rangle}\right|_{r}
$$

if $m_{j}$ et $\left(y_{0}\right)_{j}$ have opposite signs, or 


$$
|g|_{r} e^{2 \pi|m| r}=\left.\sup _{x}\left|g\left(x-i y_{0}\right)\right|\left|e^{2 i \pi\left\langle m, x-i y_{0}\right\rangle}=\right| g e^{2 i \pi\langle m, .\rangle}\right|_{r}
$$

if $m_{j}$ et $\left(y_{0}\right)_{j}$ have the same sign, whence (54) if $m$ has only one non-zero component. For $1 \leq l \leq d$, let $\bar{m}_{l}=\left(m_{1}, \ldots, m_{l}, 0, \ldots, 0\right)$. Assume that

$$
|g|_{r} e^{2 \pi|m| r}=\left|g e^{2 i \pi\left\langle\bar{m}_{j-1}, .\right\rangle}\right|_{r} e^{2 \pi\left(\left|m_{j}\right|+\cdots+\left|m_{d}\right|\right) r}
$$

and that $\left|g e^{2 i \pi\left\langle\bar{m}_{j-1}, .\right\rangle}\right|_{r}$ is reached at $\bar{y}$. Let $\delta_{j} \in\{-1,1\}$ be such that $m_{j}$ and $\delta_{j} \bar{y}_{j}$ have opposite signs. Then

$$
\begin{aligned}
|g|_{r} e^{2 \pi|m| r} & =\left|g e^{2 i \pi\left\langle\bar{m}_{j-1}, .\right\rangle}\right|_{r} e^{2 \pi\left(\left|m_{j}\right|+\cdots+\left|m_{d}\right|\right) r} \\
& =\sup _{x, y_{k}, k \neq j}\left|g\left(x+i\left(y_{1}, \ldots, \bar{y}_{j}, \ldots, y_{d}\right)\right) e^{2 i \pi\left\langle\bar{m}_{j-1}, x+i\left(y_{1}, \ldots, \bar{y}_{j}, \ldots, y_{d}\right)\right\rangle}\right| e^{2 \pi\left(\left|m_{j}\right|+\cdots+\left|m_{d}\right|\right) r} \\
& =\sup _{x, y_{k}, k \neq j}\left|g\left(x+i\left(y_{1}, \ldots, \delta_{j} \bar{y}_{j}, \ldots, y_{d}\right)\right) e^{2 i \pi\left\langle\bar{m}_{j-1}, x+i\left(y_{1}, \ldots, \delta_{j} \bar{y}_{j}, \ldots, y_{d}\right)\right\rangle} e^{2 i \pi m_{j}\left(x_{j}+i \delta_{j} \bar{y}_{j}\right)}\right| \\
& . e^{2 \pi\left(\left|m_{j+1}\right|+\cdots+\left|m_{d}\right|\right) r} \\
& =\sup _{x, y_{k}, k \neq j}\left|g\left(x+i\left(y_{1}, \ldots, \delta_{j} \bar{y}_{j}, \ldots, y_{d}\right)\right) e^{2 i \pi\left\langle\bar{m}_{j}, x+i\left(y_{1}, \ldots, \delta_{j} \bar{y}_{j}, \ldots, y_{d}\right)\right\rangle}\right| e^{2 \pi\left(\left|m_{j+1}\right|+\cdots+\left|m_{d}\right|\right) r} \\
& =\left|g e^{2 i \pi\left\langle\bar{m}_{j}, .\right\rangle}\right|_{r} e^{2 \pi\left(\left|m_{j+1}\right|+\cdots+\left|m_{d}\right|\right) r}
\end{aligned}
$$

and (54) is obtained through a simple iteration. Thus

$$
\left|f e^{2 i \pi\langle m, .\rangle}\right|_{r^{\prime}} \leq|f|_{r^{\prime}} e^{2 \pi|m| r^{\prime}} \leq C|g|_{r} e^{2 \pi|m| r}=C\left|g e^{2 i \pi\langle m, .\rangle}\right|_{r}
$$

Remark: If $f, g$ are matrix-valued trigonometric polynomials, $f=\left(f_{j, k}\right), g=\left(g_{j, k}\right)$, and $g$ has real coefficients on $\mathbb{R}^{d}$, a similar statement holds. For if

$$
|f|_{r^{\prime}}=\sup _{x,\left|y_{j}\right| \leq r^{\prime}}\|f(x+i y)\| \leq C|g|_{r}=C \sup _{x,\left|y_{j}\right| \leq r}\|g(x+i y)\|
$$

as the norm of the greatest coefficient is equivalent to the operator norm, one has

$$
\sup _{j, k}\left|f_{j, k}\right|_{r^{\prime}} \leq C C^{\prime} \sup _{j, k}\left|g_{j, k}\right|_{r}
$$

for some $C^{\prime}$ only depending on the dimension of the matrices. So from Lemma 2.7, since there exists $j_{0}, k_{0}$ such that

$$
\forall j, k,\left|f_{j, k}\right|_{r^{\prime}} \leq C C^{\prime}\left|g_{j_{0}, k_{0}}\right|_{r}
$$

then

$$
\sup _{j, k}\left|f_{j, k} e^{2 i \pi\langle m, .\rangle}\right|_{r^{\prime}} \leq C C^{\prime} \sup _{j, k}\left|g_{j, k} e^{2 i \pi\langle m, .\rangle}\right|_{r}
$$

and as the norms are equivalent, the statement also holds in operator norm:

$$
\left|f e^{2 i \pi\langle m, .\rangle}\right|_{r^{\prime}} \leq C C^{\prime \prime}\left|g e^{2 i \pi\langle m, .\rangle}\right|_{r}
$$

for some $C^{\prime \prime}$ depending only on the dimension of the matrices. 


\section{Proposition 2.8 Let}

- $N \in \mathbb{N}$,

- $\left.\left.\kappa^{\prime} \in\right] 0, \kappa\right]$,

- $\gamma \geq n(n+1)$,

- $0<r^{\prime}<r$.

Let $\tilde{A} \in \mathcal{G}$ have $D C_{\omega}^{N}\left(\kappa^{\prime}, \tau\right)$ spectrum. Let $\tilde{F} \in C_{r}^{\omega}\left(2 \mathbb{T}^{d}, \mathcal{G}\right)$ with nice periodicity properties with respect to an $\left(\tilde{A}, \kappa^{\prime}, \gamma\right)$-decomposition $\mathcal{L}$. Then equation

$$
\forall \theta \in 2 \mathbb{T}^{d}, \partial_{\omega} \tilde{X}(\theta)=[\tilde{A}, \tilde{X}(\theta)]+\tilde{F}^{N}(\theta)-\hat{\tilde{F}}(0) ; \hat{\tilde{X}}(0)=0
$$

has a solution $\tilde{X} \in C_{r^{\prime}}^{\omega}\left(2 \mathbb{T}^{d}, \mathcal{G}\right)$ such that

- if $\tilde{F}$ has nice periodicity properties with respect to $\mathcal{L}$ and $\left(m_{L}\right)$, then $\tilde{X}$ has nice periodicity properties with respect to $\mathcal{L}$ and $\left(m_{L}\right)$; in particular, if $\tilde{F}$ is defined on $\mathbb{T}^{d}$, then so is $\tilde{X}$,

- if $\Phi$ is trivial with respect to $\mathcal{L}$, then there exist $C^{\prime}, D$ depending only on $n, d, \tau$ such that

$$
\left|\Phi^{-1} \tilde{X} \Phi\right|_{r^{\prime}} \leq C^{\prime}\left(\frac{1+\left\|\tilde{A}_{\mathcal{N}}\right\|}{\left(r-r^{\prime}\right) \kappa^{\prime}}\right)^{2 n^{2} \gamma+D}\left|\Phi^{-1} \tilde{F} \Phi\right|_{r}
$$

Moreover, the truncation of $\tilde{X}$ at order $N$ is unique.

Proof: • Let $C \in G L(n, \mathbb{C})$ be such that $C^{-1} \tilde{A} C$ is in Jordan normal form. Conjugating equation (55) by $C^{-1}$, decomposing into coefficients $x_{j, k}$ of $C^{-1} \tilde{X} C$ and developing into Fourier series, one gets for all $m \in \frac{1}{\nu} \mathbb{Z}^{d}$, with $\nu=1$ or 2 according to the periodicity of $\left(C^{-1}\left(\tilde{F}^{N}-\hat{\tilde{F}}(0)\right) C\right)_{j, k}$,

$$
i\langle m, \omega\rangle \hat{x}_{j, k}(m)=\left(\tilde{\alpha}_{j}-\tilde{\alpha}_{k}\right) \hat{x}_{j, k}(m)+\delta_{1} \hat{x}_{j, k+1}(m)+\delta_{2} \hat{x}_{j-1, k}(m)+\hat{f}(m)
$$

where $\delta_{1}, \delta_{2}$ are 0 or 1 and $\hat{f}(m)$ stands for the $m$-th Fourier coefficient of the function $\left(C^{-1}\left(\tilde{F}^{N}-\hat{\tilde{F}}(0)\right) C\right)_{j, k}$.

The diophantine conditions given by Proposition 2.6 allow the existence of an analytic solution to the set of equations (57), therefore (55) has a solution $\tilde{X}$.

- Now we shall see that $\tilde{X}^{N}$ is unique. Suppose that $\tilde{X}$ and $\tilde{Y}$ are both solutions of (55). Then

$$
\partial_{\omega}(\tilde{X}-\tilde{Y})=[\tilde{A}, \tilde{X}-\tilde{Y}] ; \quad \hat{\tilde{X}}(0)-\hat{\tilde{Y}}(0)=0
$$

The diophantine conditions on $\tilde{A}$ imply that the truncation at order $N$ of any solution of (58) is constant, and condition $\hat{\tilde{X}}(0)-\hat{\tilde{Y}}(0)=0$ implies that it vanishes, so $\tilde{X}^{N}=\tilde{Y}^{N}$. 
- To check that $\tilde{X}$ is $\mathcal{G}$-valued, it is enough to show it for $\tilde{X}^{N}$, since one can assume that $\tilde{X}=\tilde{X}^{N}$.

- if $\mathcal{G}=\operatorname{gl}(n, \mathbb{C})$, this is trivial.

- if $\mathcal{G}=g l(n, \mathbb{R})$, this comes from the unicity of the solution up to order $N$, since $\tilde{X}$ and its complex conjugate are solutions of the same equation.

- if $\mathcal{G}=\operatorname{sp}(n, \mathbb{C})$, then

$$
\begin{aligned}
\forall \theta \in 2 \mathbb{T}^{d}, \partial_{\omega} J\left(\tilde{X}(\theta)^{*} J+J \tilde{X}(\theta)\right) & =-J\left(\tilde{X}(\theta)^{*} J+J \tilde{X}(\theta)\right) \tilde{A}-J \tilde{A}^{*}\left(\tilde{X}(\theta)^{*} J+J \tilde{X}(\theta)\right) \\
& =\left[\tilde{A}, J\left(\tilde{X}(\theta)^{*} J+J \tilde{X}(\theta)\right)\right]
\end{aligned}
$$

Diophantine conditions on $\tilde{A}$ imply that $\tilde{X}^{*} J+J \tilde{X}$ is constant. Condition $\hat{\tilde{X}}(0)=0$ implies that for every $\theta \in 2 \mathbb{T}^{d}, \tilde{X}(\theta)^{*} J+J \tilde{X}(\theta)=0$, so $\tilde{X}$ takes its values in $\operatorname{sp}(n, \mathbb{C})$.

- if $\mathcal{G}=u(n)$, proceed as in the $\operatorname{sp}(n, \mathbb{C})$ case, showing this time that $\tilde{X}^{*}+\tilde{X}$ is constant and thus is zero.

- if $\mathcal{G}=\operatorname{sp}(n, \mathbb{R})$ or $o(n)$, use the previous cases and the fact that $s p(n, \mathbb{R})=s p(n, \mathbb{C}) \cap$ $g l(n, \mathbb{R})$ and $o(n)=u(n) \cap g l(n, \mathbb{R})$.

- if $\mathcal{G}=\operatorname{sl}(n, \mathbb{R})$ or $\operatorname{sl}(2, \mathbb{C})$, note that the trace of $\tilde{X}$ is solution of

$$
\forall \theta \in 2 \mathbb{T}^{d}, \partial_{\omega}(\operatorname{Tr} \tilde{X}(\theta))=\operatorname{Tr}[\tilde{A}, \tilde{X}(\theta)]=\operatorname{Tr}(\tilde{A} \tilde{X}(\theta))-\operatorname{Tr}(\tilde{X}(\theta) \tilde{A})=0
$$

so it is a constant, and as $\operatorname{Tr} \hat{\tilde{X}}(0)=0$, it is identical to zero.

- As for periodicity properties, equation (55) decomposes into blocks according to $\mathcal{L}$, then into Fourier coefficients: for $0<|m| \leq N$,

$$
2 i \pi\langle m, \omega\rangle\left(P_{L}^{\mathcal{L}} \hat{\tilde{X}}(m) P_{L^{\prime}}^{\mathcal{L}}\right)=P_{L}^{\mathcal{L}} \tilde{A} P_{L}^{\mathcal{L}} \hat{\tilde{X}}(m) P_{L^{\prime}}^{\mathcal{L}}-P_{L}^{\mathcal{L}} \hat{\tilde{X}}(m) P_{L^{\prime}}^{\mathcal{L}} \tilde{A} P_{L^{\prime}}^{\mathcal{L}}+P_{L}^{\mathcal{L}} \hat{\tilde{F}}(m) P_{L^{\prime}}^{\mathcal{L}}
$$

Let $\left(m_{L}\right)$ be a family such that $\tilde{F}$ has nice periodicity properties with respect to $\mathcal{L}$ and $\left(m_{L}\right)$. If $m$ is not in $\mathbb{Z}^{d}+m_{L}-m_{L^{\prime}}$, then $P_{L}^{\mathcal{L}} \hat{\tilde{F}}(m) P_{L^{\prime}}^{\mathcal{L}}=0$ and since $\tilde{X}^{N}$ is unique, $P_{L}^{\mathcal{L}} \hat{\tilde{X}}(m) P_{L^{\prime}}^{\mathcal{L}}=0$. For $|m|>N$ one can assume $\hat{\tilde{X}}(m)=0$. Therefore $\tilde{X}$ also has nice periodicity properties with respect to $\mathcal{L}$ et $\left(m_{L}\right)$.

- Finally let us prove the estimate (56). Let $m \in \frac{1}{2} \mathbb{Z}^{d},|m| \leq N$. First we shall prove that for all $L, L^{\prime} \in \mathcal{L}$,

$$
\left\|P_{L}^{\mathcal{L}} \hat{\tilde{X}}(m) P_{L^{\prime}}^{\mathcal{L}}\right\| \leq C^{\prime} \frac{\left(1+\left\|\tilde{A}_{\mathcal{N}}\right\|\right)^{n^{2}-1}|m|^{\left(n^{2}-1\right) \tau}}{\kappa^{\prime\left(n^{2}-1\right)}}\left\|P_{L}^{\mathcal{L}} \hat{\tilde{F}}(m) P_{L^{\prime}}^{\mathcal{L}}\right\|\left(\left\|P_{L}^{\mathcal{L}}\right\|\left\|P_{L^{\prime}}^{\mathcal{L}}\right\|\right)^{n^{2}-1}
$$


where $C^{\prime}$ only depends on $n$. The proof will be inspired by [3], Lemma 2. Let $\mathcal{A}_{L, L^{\prime}}$ be the linear operator from $g l(n, \mathbb{C})$ into itself such that for all $M \in g l(n, \mathbb{C})$,

$$
\mathcal{A}_{L, L^{\prime}} M=\tilde{A} P_{L}^{\mathcal{L}} M-M P_{L^{\prime}}^{\mathcal{L}} \tilde{A}
$$

Decomposing (55) into blocks, then into Fourier series, one obtains for all $L, L^{\prime} \in \mathcal{L}$ and all $m \in \frac{1}{2} \mathbb{Z}^{d}$ such that $0<|m| \leq N$,

$$
\left(P_{L}^{\mathcal{L}} \hat{\tilde{X}}(m) P_{L^{\prime}}^{\mathcal{L}}\right)=\left(2 i \pi\langle m, \omega\rangle-\mathcal{A}_{L, L^{\prime}}\right)^{-1} P_{L}^{\mathcal{L}} \hat{\tilde{F}}(m) P_{L^{\prime}}^{\mathcal{L}}
$$

Write $\mathcal{A}_{L, L^{\prime}}$ as an $n^{2}$-dimensional matrix. Let $A_{D} \in g l\left(n^{2}, \mathbb{C}\right)$ be a diagonal matrix and $A_{N} \in \operatorname{gl}\left(n^{2}, \mathbb{C}\right)$ a nilpotent matrix such that

$$
\left(2 i \pi\langle m, \omega\rangle-\mathcal{A}_{L, L^{\prime}}\right)=A_{D}-A_{N}
$$

Then $A_{N}$ coincides with the operator

$$
A_{N}: B \mapsto\left(\tilde{A} P_{L}^{\mathcal{L}}\right)_{\mathcal{N}} B-B\left(P_{L^{\prime}}^{\mathcal{L}} \tilde{A}\right)_{\mathcal{N}}
$$

Moreover,

$$
\left(2 i \pi\langle m, \omega\rangle-\mathcal{A}_{L, L^{\prime}}\right)^{-1}=A_{D}^{-1}\left(I+A_{N} A_{D}^{-1}+\cdots+\left(A_{N} A_{D}^{-1}\right)^{n^{2}-1}\right)
$$

We will estimate $\left(2 i \pi\langle m, \omega\rangle-\mathcal{A}_{L, L^{\prime}}\right)^{-1}$, for $m \in \mathbb{Z}^{d}$ if $L=\bar{L}^{\prime}$ and $m \in \frac{1}{2} \mathbb{Z}^{d}$ if $L \neq \bar{L}^{\prime}$. Each coefficient of $A_{D}^{-1}\left(A_{N} A_{D}^{-1}\right)^{j-1}$ has the form $\frac{p}{q}$ with $|p| \leq\left\|A_{N}\right\|^{j-1}$ and $q=\beta_{1} \ldots \beta_{j}$ where $\beta_{i}$ are eigenvalues of $2 i \pi\langle m, \omega\rangle-\mathcal{A}_{L, L^{\prime}}$. Now

$$
\sigma\left(\mathcal{A}_{L, L^{\prime}}\right)=\left\{\alpha-\alpha^{\prime} \mid \alpha \in \sigma\left(\tilde{A}_{\mid L}\right), \alpha^{\prime} \in \sigma\left(\tilde{A}_{\mid L^{\prime}}\right)\right\}
$$

and for all $\alpha \in \sigma\left(\tilde{A}_{\mid L}\right), \alpha^{\prime} \in \sigma\left(\tilde{A}_{\mid L^{\prime}}\right)$,

$$
\left|\alpha-\alpha^{\prime}-2 i \pi\langle m, \omega\rangle\right| \geq \frac{\kappa^{\prime}}{|m|^{\tau}}
$$

for all $m \in \mathbb{Z}^{d}$ if $L=\bar{L}^{\prime}$ and all $m \in \frac{1}{2} \mathbb{Z}^{d}$ if $L \neq \bar{L}^{\prime}$ Thus

$$
\left\|\left(2 i \pi\langle m, \omega\rangle-\mathcal{A}_{L, L^{\prime}}\right)^{-1}\right\| \leq n^{2} 2^{n^{2}}\left(1+\left\|\tilde{A}_{\mathcal{N}}\right\|\left(\left\|P_{L}^{\mathcal{L}}\right\|+\left\|P_{L^{\prime}}^{\mathcal{L}}\right\|\right)\right)^{n^{2}-1}\left(\frac{|m|^{\tau}}{\kappa^{\prime}}\right)^{n^{2}-1}
$$

and (61) implies (60).

- The estimate (60) implies that

$\left|P_{L}^{\mathcal{L}} \tilde{X} P_{L^{\prime}}^{\mathcal{L}}\right|_{r^{\prime}} \leq C^{\prime} \frac{\left(1+\| \tilde{A}_{\mathcal{N}}||\right)^{n^{2}-1}}{\kappa^{\prime\left(n^{2}-1\right)}} \sum_{m}|m|^{\left(n^{2}-1\right) \tau}\left|P_{L}^{\mathcal{L}} \tilde{F} P_{L^{\prime}}^{\mathcal{L}}\right|_{r} e^{-2 \pi|m| r} e^{2 \pi|m| r^{\prime}}\left(|| P_{L}^{\mathcal{L}}|| \| P_{L^{\prime}}^{\mathcal{L}}||\right)^{n^{2}-1}$

where $C^{\prime}$ only depends on $n$. Now 


$$
\begin{aligned}
\sum_{m}|m|^{\left(n^{2}-1\right) \tau} e^{-2 \pi|m|\left(r-r^{\prime}\right)} & \leq C_{d} \sum_{M \geq 1} M^{\left(n^{2}-1\right) \tau+d} e^{-2 \pi M\left(r-r^{\prime}\right)} \\
& \leq C_{d} \int_{0}^{\infty} t^{\left(n^{2}-1\right) \tau+d} e^{-2 \pi t\left(r-r^{\prime}\right)} d t \leq \frac{C_{d}}{\left(2 \pi\left(r-r^{\prime}\right)\right)^{\left(n^{2}-1\right) \tau+d+1}}
\end{aligned}
$$

where $C_{d}$ only depends on $d$, so

$$
\left|P_{L}^{\mathcal{L}} \tilde{X} P_{L^{\prime}}^{\mathcal{L}}\right|_{r^{\prime}} \leq \frac{C^{\prime \prime}}{\left(r-r^{\prime}\right)^{\left(n^{2}-1\right) \tau+d+1}} \frac{\left(1+\left\|\tilde{A}_{\mathcal{N}}\right\|\right)^{n^{2}-1}}{\kappa^{\prime\left(n^{2}-1\right)}}\left|P_{L}^{\mathcal{L}} \tilde{F} P_{L^{\prime}}^{\mathcal{L}}\right|_{r}\left(\left\|P_{L}^{\mathcal{L}}||\right\| P_{L^{\prime}}^{\mathcal{L}}||\right)^{n^{2}-1}
$$

where $C^{\prime \prime}$ only depends on $n, d, \tau$.

Let $\left(m_{L}^{\prime}\right)_{L \in \mathcal{L}}$ a family of elements of $\frac{1}{2} \mathbb{Z}^{d}$ and $\Phi$ defined by

$$
\Phi=\sum_{L \in \mathcal{L}} P_{L}^{\mathcal{L}} e^{2 i \pi\left\langle m_{L}^{\prime}, \cdot\right\rangle}
$$

then

$$
\left|\Phi^{-1} \tilde{X} \Phi\right|_{r^{\prime}}=\left|\sum_{L, L^{\prime} \in \mathcal{L}} P_{L}^{\mathcal{L}} \tilde{X} e^{2 i \pi\left\langle m_{L}^{\prime}-m_{L^{\prime}}^{\prime},\right\rangle} P_{L^{\prime}}^{\mathcal{L}}\right|_{r^{\prime}}
$$

and since $\mathcal{L}$ is an $\left(\tilde{A}, \kappa^{\prime}, \gamma\right)$-decomposition, then Lemma 2.7 applied to (63) gives

$$
\left|\Phi^{-1} \tilde{X} \Phi\right|_{r^{\prime}} \leq \frac{C_{3}}{\left(r-r^{\prime}\right)^{\left(n^{2}-1\right) \tau+d+1}}\left(\frac{1+\left\|\tilde{A}_{\mathcal{N}}\right\|}{\kappa^{\prime}}\right)^{n^{2}(2 \gamma+1)} \sum_{L, L^{\prime}}\left|P_{L}^{\mathcal{L}} \Phi^{-1} \tilde{F} \Phi P_{L^{\prime}}^{\mathcal{L}}\right|_{r}
$$

where $C_{3}$ only depends on $n, d, \tau$, whence (56).

Remark: The loss of analyticity $r-r^{\prime}$ is needed in order to have good estimates of the solution. Note that when $\mathcal{G}=o(n)$ or $u(n)$, then $\tilde{A}_{\mathcal{N}}$ is zero, thus the estimate does not depend on $\tilde{A}$.

\subsection{Inductive lemma without reduction of the eigenvalues}

\subsubsection{Auxiliary lemmas}

The first lemma will be used to iterate the inductive lemma without having to perform reduction of the eigenvalues at each step, which will greatly improve the final estimates.

Lemma 2.9 Let

- $\left.\kappa^{\prime} \in\right] 0,1[, C>0$,

- $\tilde{F} \in \mathcal{G}$, 
- $\tilde{\epsilon}=\|\tilde{F}\|$,

- $\tilde{N} \in \mathbb{N}$,

- $\tilde{A} \in \mathcal{G}$ with $D C_{\omega}^{\tilde{N}}\left(\kappa^{\prime}, \tau\right)$ spectrum.

There exists a constant $c$ only depending on $n \tau$ such that if $\tilde{\epsilon}$ satisfies

$$
\tilde{\epsilon} \leq c\left(\frac{C^{\tau} \kappa^{\prime}}{1+\|\tilde{A}\|}\right)^{2 n}
$$

and

$$
\tilde{N} \leq \frac{|\log \tilde{\epsilon}|}{C}
$$

then $\tilde{A}+\tilde{F}$ has $D C_{\omega}^{\tilde{N}}\left(\frac{3 \kappa^{\prime}}{4}, \tau\right)$ spectrum.

Proof: If $\tilde{\alpha} \in \sigma(\tilde{A}+\tilde{F})$, by Lemma 4.1 given as an appendix, there exists $\alpha \in \sigma(\tilde{A})$ such that $|\alpha-\tilde{\alpha}| \leq 2 n(|| \tilde{A} \|+1) \tilde{\epsilon}^{\frac{1}{n}}$.

By assumption $\tilde{A}$ has $D C_{\omega}^{\tilde{N}}\left(\kappa^{\prime}, \tau\right)$ spectrum. Thus for all $\alpha, \beta \in \sigma(\tilde{A}+\tilde{F})$ and all $m \in$ $\mathbb{Z}^{d}, 0<|m| \leq \tilde{N}$

$$
|\alpha-\beta-2 i \pi\langle m, \omega\rangle| \geq \frac{\kappa^{\prime}}{|m|^{\tau}}-4 n(\|\tilde{A}\|+1) \tilde{\epsilon}^{\frac{1}{n}}
$$

and if $\alpha \neq \bar{\beta}$, (66) holds for every $m \in \frac{1}{2} \mathbb{Z}^{d}, 0<|m| \leq \tilde{N}$. Therefore it is enough to show that

$$
4 n \tilde{N}^{\tau}(\|\tilde{A}\|+1) \tilde{\epsilon}^{\frac{1}{n}} \leq \frac{\kappa^{\prime}}{4}
$$

Now there is a constant $c \leq 1$ which only depends on $n \tau$ such that if $\tilde{\epsilon} \leq c$, then

$$
\tilde{\epsilon}(|\log \tilde{\epsilon}|)^{n \tau} \leq \tilde{\epsilon}^{\frac{1}{2}}
$$

so if

$$
\tilde{\epsilon} \leq c\left(\frac{C^{\tau} \kappa^{\prime}}{16 n(\|\tilde{A}\|+1)}\right)^{2 n}
$$

by asumption (65), then

$$
4 n(\|\tilde{A}\|+1) \tilde{\epsilon}^{\frac{1}{n}} \tilde{N}^{\tau} \leq 4 n(\|\tilde{A}\|+1) \tilde{\epsilon}^{\frac{1}{2 n}} C^{-\tau} \leq \frac{\kappa^{\prime}}{4}
$$

which proves the Lemma.

If $G$ is compact, then by lemma 4.2, the same is true under a smallness condition which does not depend on $\tilde{A}$.

Lemma 2.10 Let 
- $\left.\kappa^{\prime} \in\right] 0,1[, C>0$,

- $\tilde{F} \in \mathcal{G}$,

- $\tilde{\epsilon}=\|\tilde{F}\|$,

- $\tilde{N} \in \mathbb{N}$,

- $\tilde{A} \in \mathcal{G}$ with $D C_{\omega}^{\tilde{N}}\left(\kappa^{\prime}, \tau\right)$ spectrum.

There exists a constant $c$ only depending on $\tau$ such that if $\tilde{\epsilon}$ satisfies

$$
\tilde{\epsilon} \leq c\left(C^{\tau} \kappa^{\prime}\right)^{2}
$$

and

$$
\tilde{N} \leq \frac{|\log \tilde{\epsilon}|}{C}
$$

then $\tilde{A}+\tilde{F}$ has $D C_{\omega}^{\tilde{N}}\left(\frac{3 \kappa^{\prime}}{4}, \tau\right)$ spectrum.

Proof: If $\tilde{\alpha} \in \sigma(\tilde{A}+\tilde{F})$, by Lemma 4.2, there exists $\alpha \in \sigma(\tilde{A})$ such that $|\alpha-\tilde{\alpha}| \leq \tilde{\epsilon}$. Since $\tilde{A}$ has $D C_{\omega}^{\tilde{N}}\left(\kappa^{\prime}, \tau\right)$ spectrum, then for all $\alpha, \beta \in \sigma(\tilde{A}+\tilde{F})$ and all $m \in \mathbb{Z}^{d}, 0<|m| \leq$ $\tilde{N}$

$$
|\alpha-\beta-2 i \pi\langle m, \omega\rangle| \geq \frac{\kappa^{\prime}}{|m|^{\tau}}-2 \tilde{\epsilon}
$$

and if $\alpha \neq \bar{\beta}$, (69) holds for every $m \in \frac{1}{2} \mathbb{Z}^{d}, 0<|m| \leq \tilde{N}$. There is a constant $c \leq 1$ which only depends on $\tau$ such that if $\tilde{\epsilon} \leq c$, then

$$
\tilde{\epsilon}(|\log \tilde{\epsilon}|)^{\tau} \leq \tilde{\epsilon}^{\frac{1}{2}}
$$

so it is enough that

$$
\tilde{\epsilon} \leq c\left(\frac{C^{\tau} \kappa^{\prime}}{8}\right)^{2}
$$

The following lemma will be used to avoid doubling the period more than once.

Lemma 2.11 Let $A, A^{\prime} \in \operatorname{gl}(n, \mathbb{R})$ and $H: 2 \mathbb{T}^{d} \rightarrow g l(n, \mathbb{R})$. Assume that $H$ has nice periodicity properties with respect to an $A$-decomposition $\mathcal{L}$ and assume

$$
\forall L, L^{\prime} \in \mathcal{L}, P_{L}^{\mathcal{L}}\left(A^{\prime}-A\right) P_{L^{\prime}}^{\mathcal{L}} \neq 0 \Rightarrow P_{L}^{\mathcal{L}} H P_{L^{\prime}}^{\mathcal{L}} \in C^{0}\left(\mathbb{T}^{d}, g l(n, \mathbb{R})\right)
$$

Then $H$ has nice periodicity properties with respect to an $A^{\prime}$-decomposition which is less fine than $\mathcal{L}$. 
Proof: Define a decomposition $\mathcal{L}^{\prime}$ of $\mathbb{C}^{n}$ as follows: for all $L, L^{\prime} \in \mathcal{L}$,

$$
\left(\exists L_{0} \in \mathcal{L}^{\prime} \mid L \subset L_{0}, L^{\prime} \subset L_{0}\right) \Leftrightarrow P_{L}^{\mathcal{L}} H P_{L^{\prime}}^{\mathcal{L}} \in C^{0}\left(\mathbb{T}^{d}, g l(n, \mathbb{R})\right)
$$

Let $\left(m_{L}\right)$ be a family such that $H$ has nice periodicity properties with respect to $\mathcal{L}$ and $\left(m_{L}\right)$. For all $L^{\prime} \in \mathcal{L}^{\prime}$, let $L$ be a subspace of $\mathcal{L}$ contained in $L^{\prime}$ and let $\bar{m}_{L^{\prime}}=m_{L}$; the class of $\bar{m}_{L^{\prime}}$ in the equivalence relation

$$
m \sim m^{\prime} \Leftrightarrow m-m^{\prime} \in \mathbb{Z}^{d}
$$

does not depend on a particular choice of $L$. Then for all $L^{\prime} \in \mathcal{L}^{\prime}$,

$$
e^{2 i \pi\left\langle\bar{m}_{\left.L^{\prime}, .\right\rangle}\right.} P_{L^{\prime}}^{\mathcal{L}^{\prime}}=\sum_{L \in \mathcal{L}, L \subset L^{\prime}} e^{2 i \pi\left\langle\bar{m}_{L^{\prime}, .}\right.} P_{L}^{\mathcal{L}}
$$

so for all $L_{1}, L_{2} \in \mathcal{L}^{\prime}$,

$$
P_{L_{1}}^{\mathcal{L}^{\prime}} H P_{L_{2}}^{\mathcal{L}^{\prime}} e^{2 i \pi\left\langle\bar{m}_{L_{1}}-\bar{m}_{L_{2}}, .\right\rangle}=\sum_{L_{1}^{\prime} \subset L_{1}, L_{2}^{\prime} \subset L_{2}} P_{L_{1}^{\prime}}^{\mathcal{L}} H P_{L_{2}^{\prime}}^{\mathcal{L}} e^{2 i \pi\left\langle m_{L_{1}^{\prime}}-m_{L_{2}^{\prime}}, .\right\rangle} e^{2 i \pi\left\langle\bar{m}_{L_{1}}-m_{L_{1}^{\prime}}-\left(\bar{m}_{L_{2}}-m_{L_{2}^{\prime}}\right) . .\right\rangle}
$$

which is continuous on $\mathbb{T}^{d}$. Moreover, let $L_{0} \in \mathcal{L}^{\prime}$, then

$$
P_{L_{0}}^{\mathcal{L}^{\prime}} H P_{\bar{L}_{0}}^{\mathcal{L}^{\prime}}=\sum_{L, L^{\prime} \in \mathcal{L}, L \subset L_{0}, L^{\prime} \subset \bar{L}_{0}} P_{L}^{\mathcal{L}} H P_{L^{\prime}}^{\mathcal{L}}
$$

which is continuous on $\mathbb{T}^{d}$. Thus $H$ has nice periodicity properties with respect to $\mathcal{L}^{\prime}$. By definition, $\mathcal{L}^{\prime}$ is $A$-invariant. Moreover, assumption (70) implies

$$
A^{\prime}-A=\sum_{L^{\prime} \in \mathcal{L}^{\prime}} P_{L^{\prime}}^{\mathcal{L}^{\prime}}\left(A^{\prime}-A\right) P_{L^{\prime}}^{\mathcal{L}^{\prime}}
$$

so it also implies that $\mathcal{L}^{\prime}$ is $A^{\prime}-A$-invariant. Thus, $\mathcal{L}^{\prime}$ is $A^{\prime}$-invariant and so it is an $A^{\prime}$-decomposition.

Here is a standard lemma on the estimate of the rest of the Fourier series for an analytic function.

Lemma 2.12 Let $H \in C_{r}^{\omega}\left(2 \mathbb{T}^{d}, g l(n, \mathbb{C})\right)$. Soit $N \in \mathbb{N}$ and $H^{N}$ the truncation of $H$ at $\operatorname{order} N$. Then for all $r^{\prime}<r$,

$$
\left|H-H^{N}\right|_{r^{\prime}} \leq \frac{C N^{d}}{\left(r-r^{\prime}\right)^{d+1}}|H|_{r} e^{-2 \pi N\left(r-r^{\prime}\right)}
$$

where $C$ only depends on $d$.

Proof: It is a simple computation. Since

$$
H-H^{N}=\sum_{|m|>N} \hat{H}(m) e^{2 i \pi\langle m, .\rangle}
$$


then

$$
\begin{aligned}
\left|H-H^{N}\right|_{r^{\prime}} & \leq \sum_{|m|>N} \| \hat{H}(m)|| e^{2 \pi|m| r^{\prime}} \leq|H|_{r} \sum_{|m|>N} e^{-2 \pi|m|\left(r-r^{\prime}\right)} \\
& \leq C|H|_{r} \sum_{M>N} M^{d} e^{-2 \pi M\left(r-r^{\prime}\right)} \leq C|H|_{r} \frac{N^{d}}{\left(r-r^{\prime}\right)^{d+1}} e^{-2 \pi N\left(r-r^{\prime}\right)}
\end{aligned}
$$

\subsubsection{Inductive lemma}

Proposition 2.13 Let

- $\tilde{\epsilon}>0, \tilde{r} \leq 1, \tilde{r}^{\prime} \in\left[\frac{\tilde{r}}{2}, \tilde{r}\left[, \kappa^{\prime}>0, \tilde{N} \in \mathbb{N}, \gamma \geq n(n+1), C>0 ;\right.\right.$

- $\tilde{F} \in C_{\tilde{r}}^{\omega}\left(2 \mathbb{T}^{d}, \mathcal{G}\right), \tilde{A} \in \mathcal{G}$,

- $\mathcal{L}$ an $\left(\tilde{A}, \kappa^{\prime}, \gamma\right)$-decomposition.

There exists a constant $C^{\prime \prime}>0$ depending only on $\tau, n$ such that if

1. $\tilde{A}$ has $D C_{\omega}^{\tilde{N}}\left(\kappa^{\prime}, \tau\right)$ spectrum;

2.

$$
\|\hat{\tilde{F}}(0)\| \leq \tilde{\epsilon} \leq C^{\prime \prime}\left(\frac{C^{\tau} \kappa^{\prime}}{1+\|\tilde{A}\|}\right)^{2 n}
$$

and

$$
\tilde{N} \leq \frac{|\log \tilde{\epsilon}|}{C}
$$

3. $\tilde{F}$ has nice periodicity properties with respect to $\mathcal{L}$

then there exist

- $C^{\prime} \in \mathbb{R}$ depending only on $n, d, \kappa, \tau$,

- $D \in \mathbb{N}$ depending only on $n, d, \tau$,

- $X \in C_{\tilde{r}^{\prime}}^{\omega}\left(2 \mathbb{T}^{d}, \mathcal{G}\right)$,

- $A^{\prime} \in \mathcal{G}$

- an $\left(A^{\prime}, \frac{3 \kappa^{\prime}}{4}, \gamma\right)$-decomposition $\mathcal{L}^{\prime}$

satisfying the following properties:

1. A' has $D C_{\omega}^{\tilde{N}}\left(\frac{3 \kappa^{\prime}}{4}, \tau\right)$ spectrum,

2. $\left\|A^{\prime}-\tilde{A}\right\| \leq \tilde{\epsilon}$; 
3. the map $F^{\prime} \in C_{\tilde{r}^{\prime}}^{\omega}\left(2 \mathbb{T}^{d}, \mathcal{G}\right)$ defined by

$$
\forall \theta \in 2 \mathbb{T}^{d}, \partial_{\omega} e^{X(\theta)}=(\tilde{A}+\tilde{F}(\theta)) e^{X(\theta)}-e^{X(\theta)}\left(A^{\prime}+F^{\prime}(\theta)\right)
$$

has nice periodicity properties with respect to $\mathcal{L}^{\prime}$

4. If $\Phi$ is trivial with respect to $\mathcal{L}$,

then

$$
\left|\Phi^{-1} X \Phi\right|_{\tilde{r}^{\prime}} \leq C^{\prime}\left(\frac{1+\left\|\tilde{A}_{\mathcal{N}}\right\|}{\kappa^{\prime}\left(\tilde{r}-\tilde{r}^{\prime}\right)}\right)^{D \gamma}\left|\Phi^{-1} \tilde{F} \Phi\right|_{\tilde{r}}
$$

5. and if $\Phi$ is trivial with respect to $\mathcal{L}$,

$$
\begin{aligned}
\left|\Phi^{-1} F^{\prime} \Phi\right|_{\tilde{r}^{\prime}} & \leq C^{\prime}\left(\frac{1+\| \tilde{A}_{\mathcal{N}}||}{\kappa^{\prime}\left(\tilde{r}-\tilde{r}^{\prime}\right)}\right)^{D \gamma} e^{\left|\Phi^{-1} X \Phi\right|_{\tilde{r}^{\prime}}\left|\Phi^{-1} \tilde{F} \Phi\right|_{\tilde{r}}} \\
& \left(|\Phi|_{\tilde{r}}^{2}\left|\Phi^{-1}\right|_{\tilde{r}}^{2} \tilde{N}^{d} e^{-2 \pi \tilde{N}\left(\tilde{r}-\tilde{r}^{\prime}\right)}+\left|\Phi^{-1} \tilde{F} \Phi\right|_{\tilde{r}^{\prime}}\left(1+e^{\left.\left.\left|\Phi^{-1} X \Phi\right|_{\tilde{r}^{\prime}}\right)\right)}\right.\right.
\end{aligned}
$$

Moreover, if $\tilde{F}$ is continuous on $\mathbb{T}^{d}$, then so are $X$ and $F^{\prime}$. If $\mathcal{G}=o(n)$ or $u(n)$, then the same holds replacing condition (172) by

$$
\|\hat{\tilde{F}}(0)\| \leq \tilde{\epsilon} \leq C^{\prime \prime}\left(C^{\tau} \kappa^{\prime}\right)^{2}
$$

Proof: By assumption, $\tilde{F}$ has nice periodicity properties with respect to $\mathcal{L}$ and some family $\left(m_{L}\right)$ and $\tilde{A}$ has $D C_{\omega}^{\tilde{N}}\left(\kappa^{\prime}, \tau\right)$ spectrum, so one can apply Proposition 2.8. Let $X \in C_{r^{\prime}}^{\omega}\left(2 \mathbb{T}^{d}, \mathcal{G}\right)$ be a solution of

$$
\forall \theta \in 2 \mathbb{T}^{d}, \partial_{\omega} X(\theta)=[\tilde{A}, X(\theta)]+\tilde{F}^{\tilde{N}}(\theta)-\tilde{\tilde{F}}(0)
$$

satisfying the conclusion of Proposition 2.8 ,

Let $A^{\prime}=\tilde{A}+\hat{\tilde{F}}(0)$. Then $A^{\prime} \in \mathcal{G}$ and $\left\|\tilde{A}-A^{\prime}\right\|=\|\hat{\tilde{F}}(0)\|$, so property 2 holds.

Moreover, let $c$ be the constant given by Lemma 2.9, and assume $C^{\prime \prime} \leq c$. Assumptions (72) and (73) make it possible to apply Lemma 2.9] and infer that $A^{\prime}$ has $D C_{\omega}^{\tilde{N}}\left(\frac{3 \kappa^{\prime}}{4}, \tau\right)$ spectrum, thus property 1 holds. If $\mathcal{G}=o(n)$ or $u(n)$, one can apply lemma 2.10 instead of lemma 2.9 to get the same result with the weaker smallness condition (77)).

Let $F^{\prime} \in C_{r^{\prime}}^{\omega}\left(2 \mathbb{T}^{d}, \mathcal{G}\right)$ the map defined in $(\mathbf{7 4})$. Then

$$
F^{\prime}=e^{-X}\left(\tilde{F}-\tilde{F}^{\tilde{N}}\right)+e^{-X} \tilde{F}\left(e^{X}-I d\right)+\left(e^{-X}-I d\right) \hat{\tilde{F}}(0)-e^{-X} \sum_{k \geq 2} \frac{1}{k !} \sum_{l=0}^{k-1} X^{l}\left(\tilde{F}^{\tilde{N}}-\hat{\tilde{F}}(0)\right) X^{k-1-l}
$$


We shall appply Lemma 2.11 with $A=\tilde{A}$ and $G=F^{\prime}$, in order to get property 3, The map $F^{\prime}$ has nice periodicity properties with respect to $\mathcal{L}$ and some family $\left(m_{L}\right)$ since $X$ and $\tilde{F}$ have them. Moreover, as $\tilde{F}$ has nice periodicity properties with respect to $\mathcal{L}$,

$$
P_{L}^{\mathcal{L}} \hat{\tilde{F}}(0) P_{L^{\prime}}^{\mathcal{L}} \neq 0 \Rightarrow P_{L}^{\mathcal{L}} \tilde{F} P_{L^{\prime}}^{\mathcal{L}} \in C^{0}\left(\mathbb{T}^{d}\right)
$$

and since

$$
P_{L}^{\mathcal{L}} \tilde{F} P_{L^{\prime}}^{\mathcal{L}} \in C^{0}\left(\mathbb{T}^{d}\right) \Rightarrow m_{L}-m_{L^{\prime}} \in \mathbb{Z}^{d} \Rightarrow P_{L}^{\mathcal{L}} F^{\prime} P_{L^{\prime}}^{\mathcal{L}} \in C^{0}\left(\mathbb{T}^{d}\right)
$$

then assumption (70) of Lemma 2.11 is fulfilled. By Lemma 2.11, $F^{\prime}$ has therefore nice periodicity properties with respect to an $A^{\prime}$-decomposition $\mathcal{L}^{\prime}$ which is less fine than $\mathcal{L}$, so $\mathcal{L}^{\prime}$ is an $\left(\tilde{A}, \kappa^{\prime}, \gamma\right)$-decomposition. As it is an $\left(\tilde{A}, \kappa^{\prime}, \gamma\right)$-decomposition, and by property 2. each subspace $L \in \mathcal{L}^{\prime}$ satisfies

$$
\left\|P_{L}^{\mathcal{L}^{\prime}}\right\| \leq C_{0}\left(\frac{1+\left\|A_{\mathcal{N}}^{\prime}\right\|+2 \tilde{\epsilon}}{\kappa^{\prime}}\right)^{\gamma} \leq C_{0}\left(\frac{1+\left\|A_{\mathcal{N}}^{\prime}\right\|}{\frac{3 \kappa^{\prime}}{4}}\right)^{\gamma}
$$

and so $\mathcal{L}^{\prime}$ is an $\left(A^{\prime}, \frac{3 \kappa^{\prime}}{4}, \gamma\right)$-decomposition, thus property 3 is satisfied.

Property 4 is given by Proposition 2.8 .

- By Lemma 2.12,

$$
\left|\tilde{F}-\tilde{F}^{\tilde{N}}\right|_{\tilde{r}^{\prime}} \leq C_{1} \tilde{N}^{d}|\tilde{F}| \tilde{r} \frac{e^{-2 \pi \tilde{N}\left(\tilde{r}-\tilde{r}^{\prime}\right)}}{\left(\tilde{r}-\tilde{r}^{\prime}\right)^{d+1}}
$$

where $C_{1}$ only depends on $d$. By (78), (56) and Lemma 2.12, it is true that

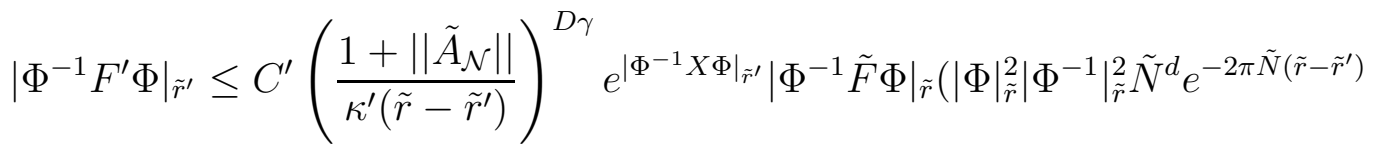

$$
\begin{aligned}
& \left.+\left|\Phi^{-1} \tilde{F} \Phi\right|_{\tilde{r}^{\prime}}\left(1+e^{\left|\Phi^{-1} X \Phi\right|_{\tilde{r}^{\prime}}}\right)\right)
\end{aligned}
$$

where $C^{\prime}$ only depends on $n, d, \kappa, \tau$ and $D$ only depends on $n, d, \tau$, whence property 5 ,

\subsection{Inductive step}

Now we are able to state the whole inductive step. In the following we will denote

$$
\left\{\begin{array}{c}
N(r, \epsilon)=\frac{1}{2 \pi r}|\log \epsilon| \\
R\left(r, r^{\prime}\right)=\frac{1}{\left(r-r^{\prime}\right)^{8}} 80^{4}\left(\frac{1}{2} n(n-1)+1\right)^{2} \\
\kappa^{\prime \prime}\left(r, r^{\prime}, \epsilon\right)=\frac{\kappa}{n\left(8 R\left(r, r^{\prime}\right)^{\frac{1}{2} n(n-1)+1} N(r, \epsilon)\right)^{\tau}}
\end{array}\right.
$$

Proposition 2.14 Let

- $A \in \mathcal{G}$, 
- $r \leq \frac{1}{2}, r^{\prime \prime} \in\left[\frac{95}{96} r, r[, \gamma \geq n(n+1)\right.$,

- $\bar{A}, \bar{F} \in C_{r}^{\omega}\left(2 \mathbb{T}^{d}, \mathcal{G}\right)$ and $\Psi \in C_{r}^{\omega}\left(2 \mathbb{T}^{d}, G\right)$,

- $\epsilon=|\bar{F}|_{r}$,

There exists $\tilde{C}^{\prime}>0$ depending only on $n, d, \kappa, \tau, \gamma$ and there exists $D_{3} \in \mathbb{N}$ depending only on $n, d, \tau$ such that if

1. $\bar{A}$ is reducible to $A$ by $\Psi$,

2. $\Psi^{-1} \bar{F} \Psi$ has nice periodicity properties with respect to an $\left(A, \kappa^{\prime \prime}\left(r, r^{\prime \prime}, \epsilon\right), \gamma\right)$-decomposition $\mathcal{L}$

3.

$$
\epsilon \leq \frac{\tilde{C}^{\prime}}{(\|A\|+1)^{D_{3} \gamma}}\left(r-r^{\prime \prime}\right)^{D_{3} \gamma}
$$

4. $|\Psi|_{r} \leq\left(\frac{1}{\epsilon}\right)^{-\frac{1}{2}\left(r-r^{\prime \prime}\right)}$ et $\left|\Psi^{-1}\right|_{r} \leq\left(\frac{1}{\epsilon}\right)^{-\frac{1}{2}\left(r-r^{\prime \prime}\right)}$,

then there exist

- $\epsilon^{\prime} \in\left[\epsilon^{R\left(r, r^{\prime \prime}\right)^{n^{2}}}, \epsilon^{100}\right]$;

- $Z^{\prime} \in C_{r^{\prime \prime}}^{\omega}\left(2 \mathbb{T}^{d}, G\right)$,

- $\bar{A}^{\prime}, \bar{F}^{\prime} \in C_{r^{\prime \prime}}^{\omega}\left(2 \mathbb{T}^{d}, \mathcal{G}\right)$,

- $\Psi^{\prime} \in C_{r}^{\omega}\left(2 \mathbb{T}^{d}, G\right)$,

- $A^{\prime} \in \mathcal{G}$

satisfying the following properties:

1. $\bar{A}^{\prime}$ is reducible by $\Psi^{\prime}$ to $A^{\prime}$,

2. the map $\Psi^{\prime-1} \bar{F}^{\prime} \Psi^{\prime}$ has nice periodicity properties with respect to an $\left.\left(A^{\prime}, \kappa^{\prime \prime}\left(r^{\prime \prime}, r^{\prime \prime}-\frac{r-r^{\prime \prime}}{2}, \epsilon^{\prime}\right), 2 \gamma\right)\right)$ decomposition $\mathcal{L}^{\prime}$

3. $\left|\bar{F}^{\prime}\right|_{r^{\prime \prime}} \leq \epsilon^{\prime}$,

4. $\left|\Psi^{\prime}\right|_{r^{\prime \prime}} \leq\left(\frac{1}{\epsilon^{\prime}}\right)^{\frac{1}{4}\left(r-r^{\prime \prime}\right)}$ and $\left|\Psi^{\prime-1}\right|_{r^{\prime \prime}} \leq\left(\frac{1}{\epsilon^{\prime}}\right)^{\frac{1}{4}\left(r-r^{\prime \prime}\right)}$,

5. $\left\|A^{\prime}\right\| \leq\|A\|+|\log \epsilon|\left(\frac{1}{r-r^{\prime}}\right)^{D_{3}}$;

6.

$$
\partial_{\omega} Z^{\prime}=(\bar{A}+\bar{F}) Z^{\prime}-Z^{\prime}\left(\bar{A}^{\prime}+\bar{F}^{\prime}\right)
$$

7.

$$
\left|Z^{\prime}-I d\right|_{r^{\prime \prime}} \leq \frac{1}{\tilde{C}^{\prime}}\left(\frac{(1+|| A||)|\log \epsilon|}{r-r^{\prime \prime}}\right)^{D_{3} \gamma} \epsilon^{1-4\left(r-r^{\prime \prime}\right)}
$$

and so does $\left(Z^{\prime}\right)^{-1}-I d$. 


\section{Moreover,}

- in dimension 2, if $\bar{A}, \bar{F}$ are continuous on $\mathbb{T}^{d}$, and if assumption 2 is replaced by

2]'. $\Psi$ is such that for all function $H$ continuous on $\mathbb{T}^{d}, \Psi H \Psi^{-1}$ is continuous on $\mathbb{T}^{d}$,

then $Z^{\prime}, \bar{A}^{\prime}, \bar{F}^{\prime}$ are continuous on $\mathbb{T}^{d}$ and property 0 is replaced by

2]'. $\Psi^{\prime}$ is such that for every function $H$ continuous on $\mathbb{T}^{d}, \Psi^{\prime} H \Psi^{-1}$ is continuous on $\mathbb{T}^{d}$.

- If $\mathcal{G}=\operatorname{gl}(n, \mathbb{C})$ or $u(n)$ and if $\bar{A}, \bar{F}, \Psi$ are continuous on $\mathbb{T}^{d}$, then $Z^{\prime}, \bar{A}^{\prime}, \bar{F}^{\prime}, \Psi^{\prime}$ are continuous on $\mathbb{T}^{d}$.

- if $\mathcal{G}=o(n)$ or $u(n)$, the same holds with the weaker condition

$$
\epsilon \leq \tilde{C}^{\prime}\left(r-r^{\prime \prime}\right)^{D_{3} \gamma}
$$

instead of (80);

- if $\mathcal{G}=\operatorname{sl}(2, \mathbb{C})$ or $\operatorname{sl}(2, \mathbb{R})$, then either $\Psi^{\prime-1} \Psi$ is the identity or $\left\|A^{\prime}\right\| \leq \kappa^{\prime \prime}\left(r, r^{\prime \prime}, \epsilon\right)+$ $\epsilon^{\frac{1}{2}}$.

The proof will be made in two steps: the first step is to reduce the perturbation when there are resonances. The second step is to iterate Proposition 2.13 as many times as possible using the fact that resonances, once removed, do not reappear immediately.

First step: removing the resonances Let $r^{\prime}=\frac{r+r^{\prime \prime}}{2}$. Let $R=R\left(r, r^{\prime}\right) ; N=$ $N(r, \epsilon) ; \kappa^{\prime \prime}=\kappa^{\prime \prime}\left(r, r^{\prime}, \epsilon\right)$. Let $\bar{N}$ be given by Proposition 2.6] and $\Phi$ a map of reduction of the eigenvalues of $A$ at order $R, \bar{N}$. Let $\Psi^{\prime}=\Psi \Phi$ and $\tilde{F}=\left(\Psi^{\prime}\right)^{-1} \bar{F} \Psi^{\prime}$.

We shall apply Proposition 2.13 with

$$
\tilde{\epsilon}=\epsilon^{1-2\left(r-r^{\prime}\right)-\frac{1}{48}}, \tilde{r}=r, \tilde{r}^{\prime}=r^{\prime}, \kappa^{\prime}=\frac{\kappa^{\prime \prime}}{C_{0}}, \tilde{N}=R \bar{N}, C=\frac{2 \pi r}{R^{\frac{1}{2} n(n-1)+1}}
$$

and $\tilde{A} \in \mathcal{G}$ such that

$$
\forall \theta \in 2 \mathbb{T}^{d}, \partial_{\omega} \Phi(\theta)=A \Phi(\theta)-\Phi(\theta) \tilde{A}
$$

Let $C^{\prime \prime}$ be given by Proposition 2.13 (depending only on $n$ and $\tau$ ).

The matrix $\tilde{A}$ has $D C_{\omega}^{R \bar{N}}\left(\kappa^{\prime \prime}, \tau\right)$ spectrum. By assumption, $\Psi^{-1} \bar{F} \Psi$ has nice periodicity properties with respect to an $\left(A, \kappa^{\prime \prime}, \gamma\right)$-decomposition $\mathcal{L}$ and some family $\left(m_{L}\right)$. Moreover 
$\Phi$ is trivial with respect to $\mathcal{L}_{A, \kappa^{\prime \prime}}$. Since $\mathcal{L}$ is an $A$-decomposition, there is a Jordan decomposition which is finer than $\mathcal{L}$; and since $\mathcal{L}_{A, \kappa^{\prime \prime}}$ is less fine than any Jordan decomposition, one can define an $A$-decomposition $\overline{\mathcal{L}}$ in the following way:

$$
L \in \overline{\mathcal{L}} \Leftrightarrow \exists L_{1} \in \mathcal{L}, L_{2} \in \mathcal{L}_{A, \kappa^{\prime \prime}} \mid L=L_{1} \cap L_{2}
$$

$\overline{\mathcal{L}}$ is an $\left(A, \frac{\kappa^{\prime \prime}}{C_{0}}, 2 \gamma\right)$-decomposition since $\mathcal{L}$ and $\mathcal{L}_{A, \kappa^{\prime \prime}}$ are $\left(A, \kappa^{\prime \prime}, \gamma\right)$-decompositions and $\tilde{F}$ has nice periodicity properties with respect to $\overline{\mathcal{L}}$. Since $\overline{\mathcal{L}}$ is an $\left(A, \frac{\kappa^{\prime \prime}}{C_{0}}, 2 \gamma\right)$-decomposition, it is also an $\left(\tilde{A}, \frac{\kappa^{\prime \prime}}{C_{0}}, 2 \gamma\right)$-decomposition (because the nilpotent parts of $A$ and $\tilde{A}$ coincide, and because any Jordan decomposition for $A$ is a Jordan decomposition for $\tilde{A}$ ). Moreover,

$$
\|\hat{\tilde{F}}(0)\| \leq|\tilde{F}|_{0} \leq|\Phi|_{0}\left|\Phi^{-1}\right|_{0}|\Psi|_{0}\left|\Psi^{-1}\right|_{0}|\bar{F}|_{0}
$$

Now by (47), for all $s^{\prime} \geq 0$,

$$
|\Phi|_{s^{\prime}} \leq C_{0}\left(\frac{1+\left\|A_{\mathcal{N}}\right\|}{\kappa^{\prime \prime}}\right)^{n(n+1)} e^{4 \pi \bar{N} s^{\prime}}
$$

and so does $\Phi^{-1}$. Thus

$$
\|\hat{\tilde{F}}(0)\| \leq \epsilon^{1-2\left(r-r^{\prime}\right)} C_{0}^{2}\left(\frac{1+\left\|A_{\mathcal{N}}\right\|}{\kappa^{\prime \prime}}\right)^{2 n(n+1)}
$$

therefore, if $\tilde{C}^{\prime} \leq C_{0}^{96}$ and $D_{3} \gamma \geq 96 n(n+1)$, then

$$
\|\hat{\tilde{F}}(0)\| \leq \epsilon^{1-2\left(r-r^{\prime}\right)-\frac{1}{48}}
$$

Assumption (80), which implies (172) with

$$
\tilde{C}^{\prime} \leq C^{\prime \prime 4}\left(\frac{C}{\left(r-r^{\prime}\right)^{4 n(n-1)+9}}\right)^{8 n \tau}, D_{3} \gamma \geq 64 n(n(n-1)+2) \tau
$$

(note that $\frac{C}{\left(r-r^{\prime}\right)^{4 n(n-1)+9}}$ has a lower bound which is independent of $r-r^{\prime}$ ), together with the choice of $\tilde{N}$ which implies (73) , make it possible to apply Proposition 2.13 to obtain $C^{\prime}>0$ depending only on $n, d, \kappa, \tau, D \in \mathbb{N}$ depending only on $n, d, \tau$ and functions $X \in C_{r^{\prime}}^{\omega}\left(2 \mathbb{T}^{d}, \mathcal{G}\right), F_{1} \in C_{r^{\prime}}^{\omega}\left(2 \mathbb{T}^{d}, \mathcal{G}\right)$, and a matrix $A_{1} \in \mathcal{G}$ such that

- $A_{1}$ has $D C_{\omega}^{R \bar{N}}\left(\frac{3}{4}\left(\frac{\kappa^{\prime \prime}}{C_{0}}\right), \tau\right)$ spectrum

- $\left\|A_{1}-\tilde{A}\right\| \leq \epsilon^{\frac{23}{24}}$, which implies

$$
\left\|A_{1}-A\right\| \leq\left\|A_{1}-\tilde{A}\right\|+\|A-\tilde{A}\| \leq \epsilon^{\frac{23}{24}}+4 \pi \bar{N}
$$

If $\mathcal{G}=\operatorname{sl}(2, \mathbb{C})$ or $\operatorname{sl}(2, \mathbb{R})$, then

$$
\left\|A_{1}\right\| \leq\|\tilde{A}\|+\epsilon^{\frac{23}{24}} \leq \kappa^{\prime \prime}+\epsilon^{\frac{23}{24}}
$$


- $\partial_{\omega} e^{X}=(\tilde{A}+\tilde{F}) e^{X}-e^{X}\left(A_{1}+F_{1}\right)$,

- $F_{1}$ has nice periodicity properties with respect to an $\left(A_{1}, \frac{3 \kappa^{\prime \prime}}{4 C_{0}}, 2 \gamma\right)$-decomposition $\mathcal{L}^{\prime}$

- and since $\Phi$ is trivial with respect to $\overline{\mathcal{L}}$,

$$
\left|\Phi X \Phi^{-1}\right|_{r^{\prime}} \leq C^{\prime}\left(\frac{C_{0}\left(1+\| A_{\mathcal{N}}||\right)}{\kappa^{\prime \prime}\left(r-r^{\prime}\right)}\right)^{D \gamma}\left|\Phi \tilde{F} \Phi^{-1}\right|_{r}
$$

and

$$
\begin{aligned}
& \left|\Phi F_{1} \Phi^{-1}\right|_{r^{\prime}} \leq C^{\prime}\left(\frac{C_{0}\left(1+\| A_{\mathcal{N}}||\right)}{\kappa^{\prime \prime}\left(r-r^{\prime}\right)}\right)^{D \gamma} e^{\left|\Phi X \Phi^{-1}\right|_{r^{\prime}}}\left|\Phi \tilde{F} \Phi^{-1}\right|_{r}\left(|\Phi|_{r}^{2}\left|\Phi^{-1}\right|_{r}^{2}(R \bar{N})^{d} e^{-2 \pi R \bar{N}\left(r-r^{\prime}\right)}\right. \\
& +\left|\Phi \tilde{F} \Phi^{-1}\right|_{r^{\prime}}\left(1+e^{\left.\left|\Phi X \Phi^{-1}\right|_{r^{\prime}}\right)}\right)
\end{aligned}
$$

Now

$$
\left|\Phi \tilde{F} \Phi^{-1}\right|_{r} \leq|\Psi|_{r}\left|\Psi^{-1}\right|_{r}|\bar{F}|_{r} \leq \epsilon^{1-2\left(r-r^{\prime}\right)}
$$

so, by (80), if $D_{3}$ is great enough as a function of $n, \gamma, D$, then

$$
\left|\Phi F_{1} \Phi^{-1}\right|_{r^{\prime}} \leq \epsilon^{-\frac{1}{96}} \epsilon^{1-2\left(r-r^{\prime}\right)}\left((R \bar{N})^{d} \epsilon^{100}+\epsilon^{1-2\left(r-r^{\prime}\right)}\right)
$$

There exists a constant $c_{d}$ which only depends on $D, \gamma, \tau$ such that if $\epsilon \leq c_{d}$, then

$$
\epsilon^{\frac{1}{2}}|\log \epsilon|^{D \gamma \tau} \leq 1
$$

thus if $\tilde{C}^{\prime}$ is small enough and $D_{3}$ big enough (as a function of $n, d, \gamma, \tau$ ),

$$
\left|\Phi F_{1} \Phi^{-1}\right|_{r^{\prime}} \leq \epsilon^{2-4\left(r-r^{\prime}\right)-\frac{1}{96}}
$$

The estimate (84), the assumption (80) and the fact that $\left\|A_{\mathcal{N}}\right\| \leq\|A\|$, imply that

$$
|\Psi \Phi|_{r} \leq|\Psi|_{r}|\Phi|_{r} \leq \epsilon^{-\left(r-r^{\prime}\right)-\frac{1}{96}} e^{4 \pi r \bar{N}}
$$

We shall estimate $\left|\Psi \Phi e^{X}(\Psi \Phi)^{-1}-I d\right|_{r^{\prime}}$. The estimate (75) implies

$$
\left|\Phi e^{X} \Phi^{-1}-I d\right|_{r^{\prime}} \leq C^{\prime \prime}\left(\frac{\left(1+\| A_{\mathcal{N}}||\right) R^{\frac{1}{2}(n(n-1)+1) \tau} N^{\tau}}{r-r^{\prime}}\right)^{D \gamma}\left|\Phi \tilde{F} \Phi^{-1}\right|_{r}
$$

for some $C^{\prime \prime}$ only depending on $n, d, \kappa, \tau$, so

$$
\left|\Psi \Phi e^{X}(\Psi \Phi)^{-1}-I d\right|_{r^{\prime}} \leq C_{3}\left(\frac{\left(1+|| A_{\mathcal{N}}||\right)|\log \epsilon|}{r-r^{\prime}}\right)^{D_{1}^{\prime} \gamma}|\bar{F}|_{r}\left(\frac{1}{\epsilon}\right)^{4\left(r-r^{\prime}\right)}
$$


for some $C_{3}$ depending only on $n, d, \kappa, \tau$ and $D_{1}^{\prime}$ depending only on $n, d, \tau$. The same estimate holds for $\left|\Psi \Phi e^{-X}(\Psi \Phi)^{-1}-I d\right|_{r^{\prime}}$.

Let $\bar{F}_{1}=\Psi \Phi F_{1}(\Psi \Phi)^{-1}$ and let $\bar{A}_{1} \in C_{r}^{\omega}\left(2 \mathbb{T}^{d}, \mathcal{G}\right)$ such that

$$
\partial_{\omega} \Psi \Phi=\bar{A}_{1} \Psi \Phi-\Psi \Phi A_{1}
$$

Thus we have obtained

- $\bar{N} \in\left[N, R^{\frac{1}{2} n(n-1)} N\right]$,

- $Z_{1}, \Psi^{\prime} \in C_{r^{\prime}}^{\omega}\left(2 \mathbb{T}^{d}, G\right)$,

- $A_{1} \in \mathcal{G}$

- $\bar{A}_{1} \in C_{r^{\prime}}^{\omega}\left(2 \mathbb{T}^{d}, \mathcal{G}\right)$

- and $F_{1}=\left(\Psi^{\prime}\right)^{-1} \bar{F}_{1} \Psi^{\prime}$

such that

1. $\bar{A}_{1}$ is reducible to $A_{1}$ by $\Psi^{\prime}$

2. $F_{1}$ has nice periodicity properties with respect to an $\left(A_{1}, \frac{3 \kappa^{\prime \prime}}{4 C_{0}}, 2 \gamma\right)$-decomposition $\mathcal{L}_{1}$

3. $\left|\Psi^{\prime}\right|_{r^{\prime}} \leq \epsilon^{-\left(r-r^{\prime}\right)-\frac{1}{96}} e^{4 \pi r \bar{N}}$ and $\left|\Psi^{\prime-1}\right|_{r^{\prime}} \leq \epsilon^{-\left(r-r^{\prime}\right)-\frac{1}{96}} e^{4 \pi r \bar{N}}$

4. $A_{1}$ has $D C_{\omega}^{R \bar{N}}\left(\frac{3}{4} \kappa^{\prime \prime}, \tau\right)$ spectrum,

5. $\partial_{\omega} Z_{1}=(\bar{A}+\bar{F}) Z_{1}-Z_{1}\left(\bar{A}_{1}+\bar{F}_{1}\right)$

6. $\left\|A_{1}\right\| \leq\|A\|+\epsilon^{\frac{23}{24}}+4 \pi \bar{N}$, and, if $\mathcal{G}=\operatorname{sl}(2, \mathbb{C})$ or $\operatorname{sl}(2, \mathbb{R})$ and $\Psi^{\prime-1} \Psi$ is not the identity, $\left\|A_{1}\right\| \leq \kappa^{\prime \prime}\left(r, r^{\prime \prime}, \epsilon\right)+\epsilon^{\frac{23}{24}}$;

7.

$$
\left|Z_{1}-I d\right|_{r^{\prime}} \leq \frac{1}{\tilde{C}^{\prime}}\left(\frac{\left(1+|| A_{\mathcal{N}}||\right)|\log \epsilon|}{r-r^{\prime}}\right)^{D_{1} \gamma} \epsilon^{1-4\left(r-r^{\prime}\right)}
$$

and so does $\left|Z_{1}^{-1}-I d\right|_{r^{\prime}}$

8.

$$
\left|\Psi^{-1} \bar{F}_{1} \Psi\right|_{r^{\prime}} \leq \epsilon^{\frac{3}{2}}
$$

9. $\Psi^{\prime-1} \Psi$ is trivial with respect to $\mathcal{L}_{A, \kappa^{\prime \prime}}$,

10. and for every $s^{\prime} \geq 0$,

$$
\left|\Psi^{\prime-1} \Psi\right|_{s^{\prime}} \leq C_{n}\left(\frac{1+\| A_{\mathcal{N}}||}{\kappa^{\prime \prime}}\right)^{n(n+1)} e^{4 \pi \bar{N} s^{\prime}}
$$

and so does $\left|\Psi^{-1} \Psi^{\prime}\right|_{s^{\prime}}$, where $C_{n}$ only depends on $n$. 
Second step: iteration far from resonances Let $l$ such that

$$
\epsilon^{\left(\frac{4}{3}\right)^{l+1}} \leq e^{-2 \pi\left(r-r^{\prime \prime}\right) \sqrt[4]{R} \bar{N}} \leq \epsilon^{\left(\frac{4}{3}\right)^{l}}
$$

Let $\epsilon^{\prime}=e^{-2 \pi\left(r-r^{\prime \prime}\right) \sqrt[4]{R} \bar{N}}$. Define the sequence $\epsilon_{j}=\epsilon^{\left(\frac{3}{2}\right)^{j}-\frac{1}{48}}$. We shall iterate $l-1$ times Proposition 2.13, starting with $j=2$, with

- $\tilde{\epsilon}=\epsilon_{j-1}$

- $C=\left(\frac{r-r^{\prime \prime}}{160\left(\frac{1}{2} n(n-1)+1\right)}\right)^{8\left(\frac{1}{2} n(n-1)+1\right)}$

- $\tilde{r}=r_{j-2}=\frac{r+r^{\prime \prime}}{2}-(j-2) \frac{r-r^{\prime \prime}}{2 l}$,

- $\tilde{r}^{\prime}=r_{j-1}=\frac{r+r^{\prime \prime}}{2}-(j-1) \frac{r-r^{\prime \prime}}{2 l}$,

- $\kappa^{\prime}=\left(\frac{3}{4}\right)^{j-1} \frac{\kappa^{\prime \prime}}{C_{0}}$,

- $\tilde{N}=R \bar{N}$,

- $\tilde{F}=F_{j-1}$,

- $\tilde{A}=A_{j-1}$,

- $\Phi=\Psi^{-1} \Psi^{\prime}$

- $\mathcal{L}=\mathcal{L}_{1}$,

Note that for every $j$,

$$
\epsilon_{j} \leq C^{\prime \prime}\left(\frac{C^{\tau}\left(\frac{3}{4}\right)^{j} \frac{\kappa^{\prime \prime}}{C_{0}}}{1+\left\|A_{1}\right\|+\sum_{l=1}^{j-1} \epsilon_{l}}\right)^{2 n}
$$

Estimates (88) and (89) imply

$$
\left\|\hat{F}_{1}(0)\right\| \leq\left|F_{1}\right|_{0} \leq\left|\Psi^{\prime-1} \Psi\right|_{0}\left|\Psi^{-1} \Psi^{\prime}\right|_{0}\left|\Psi^{-1} \bar{F}_{1} \Psi\right|_{0} \leq C_{n}^{2}\left(\frac{1+\left\|A_{\mathcal{N}}\right\|}{\kappa^{\prime \prime}}\right)^{2 n(n+1)} \epsilon^{\frac{3}{2}} \leq \epsilon^{\frac{3}{2}-\frac{1}{48}}
$$

Moreover, $A_{1}$ has $D C_{\omega}^{R \bar{N}}\left(\frac{3}{4} \kappa^{\prime \prime}, \tau\right)$ spectrum and $F_{1}$ has nice periodicity properties with respect to $\mathcal{L}$. Let $C^{\prime \prime}$ be the constant given by Proposition 2.13. By assumption on $\epsilon$, with $C^{\prime}$ depending only on $n, d, \kappa, \tau$ and $D_{3}$ depending only on $n, \tau$, one has

$$
\tilde{\epsilon} \leq \frac{C^{\prime \prime}}{\left(1+\| A_{1}||\right)^{2 n}}\left(\frac{3 \kappa^{\prime \prime}}{4 C_{0}}\right)^{2 n} C^{2 n \tau}
$$

Moreover,

$$
R \bar{N} \leq R^{n_{0}+1} N \leq \frac{1}{C}|\log \epsilon|
$$


so the assumptions (72) and (173) of Proposition 2.13 hold with $\tilde{F}=F_{1}, \kappa^{\prime}=\kappa^{\prime \prime}, \tilde{N}=R \bar{N}$.

Fix $j$ and assume $A_{j-1}$ has $D C_{\omega}^{\tilde{N}}\left(\kappa^{\prime}, \tau\right)$ spectrum, $F_{j-1}$ has nice periodicity properties with respect to an $\left(A_{j-1},\left(\frac{3}{4}\right)^{j-1} \frac{\kappa^{\prime \prime}}{C_{0}}, 2 \gamma\right)$-decomposition,

$$
\left\|\hat{F}_{j-1}(0)\right\| \leq \epsilon_{j-1}
$$

and

$$
C R \bar{N} \leq\left|\log \epsilon_{j-1}\right|
$$

One obtains functions $F_{j}, X_{j}$ and a matrix $A_{j}$ such that

1. $A_{j}$ has $D C^{R \bar{N}}\left(\left(\frac{3}{4}\right)^{j} \frac{\kappa^{\prime \prime}}{C_{0}}, \tau\right)$ spectrum,

2. $\left\|A_{j}\right\| \leq\left\|A_{j-1}\right\|+\epsilon_{j-1}$,

3.

$$
\partial_{\omega} e^{X_{j}}=\left(A_{j-1}+F_{j-1}\right) e^{X_{j}}-e^{X_{j}}\left(A_{j}+F_{j}\right)
$$

and $F_{j}$ has nice periodicity properties with respect to an $\left(A_{j},\left(\frac{3}{4}\right)^{j} \frac{\kappa^{\prime \prime}}{C_{0}}, 2 \gamma\right)$-decomposition

4.

$$
\left|\Psi^{-1} \Psi^{\prime} X_{j} \Psi^{\prime-1} \Psi\right|_{r_{j-1}} \leq C^{\prime}\left(\frac{1+||\left(A_{j-1}\right)_{\mathcal{N}} \|}{\kappa^{\prime \prime}\left(r_{j-2}-r_{j-1}\right)}\right)^{D \gamma}\left|\Psi^{-1} \Psi^{\prime} F_{j} \Psi^{\prime-1} \Psi\right|_{r_{j-1}}
$$

for some $C^{\prime}$ depending only on $n, d, \kappa, \tau$ and some $D$ depending only on $n, d, \tau$,

5. and

$$
\begin{aligned}
\left|\Psi^{-1} \Psi^{\prime} F_{j} \Psi^{\prime-1} \Psi\right|_{r_{j-1}} & \leq C^{\prime}\left(\frac{1+||\left(A_{j-1}\right)_{\mathcal{N}}||}{\kappa^{\prime \prime}\left(r_{j-2}-r_{j-1}\right)}\right)^{D \gamma} e^{\left|\Psi^{-1} \Psi^{\prime} X_{j-1} \Psi^{\prime-1} \Psi\right|_{r_{j-2}}\left|\Psi^{-1} \Psi^{\prime} F_{j-1} \Psi^{\prime-1} \Psi\right|_{r_{j-2}}} \\
& \left(\left|\Psi^{\prime-1} \Psi\right|_{r_{j-2}}^{4}(R \bar{N})^{d} e^{-2 \pi R \bar{N}\left(r_{j-2}-r_{j-1}\right)}\right. \\
& \left.+\left(1+2 e^{\left|\Psi^{-1} \Psi^{\prime} X_{j-1} \Psi^{\prime-1} \Psi\right|_{r_{j-2}}}\right)\left|\Psi^{-1} \Psi^{\prime} F_{j-1} \Psi^{\prime-1} \Psi\right|_{r_{j-2}}\right)
\end{aligned}
$$

We shall bound $\left\|\hat{F}_{j}(0)\right\|$ to iterate Proposition 2.13. Estimates (88) and (80) imply

$$
\begin{aligned}
\left|\Psi^{-1} \Psi^{\prime} F_{j} \Psi^{\prime-1} \Psi\right|_{r_{j-1}} & \leq 3 C^{\prime}\left(\frac{1+\|\left(A_{j-1}\right)_{\mathcal{N}}||}{\kappa^{\prime \prime}\left(r_{j-2}-r_{j-1}\right)}\right)^{D \gamma}\left|\Psi^{-1} \Psi^{\prime} F_{j-1} \Psi^{\prime-1} \Psi\right|_{r_{j-2}} \\
& \left(\left|\Psi^{\prime-1} \Psi\right|_{r_{j-2}}^{4}(R \bar{N})^{d} e^{-2 \pi R \bar{N}\left(r_{j-2}-r_{j-1}\right)}+\left|\Psi^{-1} \Psi^{\prime} F_{j-1} \Psi^{\prime-1} \Psi\right|_{r_{j-2}}\right)
\end{aligned}
$$

and since $r_{j-2}-r_{j-1}=\frac{r-r^{\prime \prime}}{2 l}$, 


$$
\begin{aligned}
\left|\Psi^{-1} \Psi^{\prime} F_{j} \Psi^{\prime-1} \Psi\right|_{r_{j-1}} & \leq\left|\Psi^{-1} \Psi^{\prime} F_{j-1} \Psi^{\prime-1} \Psi\right|_{r_{j-2}}^{\frac{3}{4}}\left(\left|\Psi^{\prime-1} \Psi\right|_{r_{j-2}}^{4}(R \bar{N})^{d} e^{-2 \pi \frac{R \bar{N}\left(r-r^{\prime \prime}\right)}{2 l}}+\left|\Psi^{-1} \Psi^{\prime} F_{j-1} \Psi^{\prime-1} \Psi\right|_{r_{j-2}}\right) \\
& \leq\left|\Psi^{-1} \Psi^{\prime} F_{j-1} \Psi^{\prime-1} \Psi\right|_{r_{j-2}}^{\frac{3}{4}}\left(\left|\Psi^{\prime-1} \Psi\right|_{r_{j-2}}^{4}(R \bar{N})^{d} \epsilon^{\frac{R^{\frac{3}{4}}}{2 l}}+\left|\Psi^{-1} \Psi^{\prime} F_{j-1} \Psi^{\prime-1} \Psi\right|_{r_{j-2}}\right)
\end{aligned}
$$

Now $l$ is bounded by

$$
l \leq 8\left(\frac{1}{2} n(n-1)+1\right) \sqrt[4]{R}
$$

Moreover

$$
\left|\Psi^{\prime-1} \Psi\right|_{r_{j-2}} \leq C_{n}\left(\frac{1+\|A\|}{\kappa^{\prime \prime}}\right)^{n(n+1)} e^{4 \pi \bar{N} r_{j-2}} \leq C_{n}\left(\frac{1+\|A\|}{\kappa^{\prime \prime}}\right)^{n(n+1)} \epsilon^{\prime-\frac{2 r_{j-2}}{\left(r-r^{\prime \prime}\right) \sqrt[4]{R}}}
$$

and so

$$
\begin{aligned}
\left|\Psi^{-1} \Psi^{\prime} F_{j} \Psi^{\prime-1} \Psi\right|_{r_{j-1}} & \leq\left|\Psi^{-1} \Psi^{\prime} F_{j-1} \Psi^{\prime-1} \Psi\right|_{r_{j-2}}^{\frac{3}{4}}\left(\epsilon^{\prime}+\left|\Psi^{-1} \Psi^{\prime} F_{j-1} \Psi^{\prime-1} \Psi\right|_{r_{j-2}}\right) \\
& \leq\left|\Psi^{-1} \Psi^{\prime} F_{j-1} \Psi^{\prime-1} \Psi\right|_{r_{j-2}}^{\frac{3}{2}}
\end{aligned}
$$

By a simple induction, for every $j$,

$$
\left|\Psi^{-1} \Psi^{\prime} F_{j} \Psi^{\prime-1} \Psi\right|_{r_{j-1}} \leq\left|\Psi^{-1} \Psi^{\prime} F_{1} \Psi^{\prime-1} \Psi\right|_{r_{0}}^{\left(\frac{3}{2}\right)^{j-1}} \leq \epsilon^{\left(\frac{3}{2}\right)^{j}}
$$

Finally

$$
\left\|\hat{F}_{j}(0)\right\| \leq\left|\Psi^{-1} \Psi^{\prime} F_{j} \Psi^{\prime-1} \Psi\right|_{r_{j-1}}\left\|\Psi^{-1} \Psi^{\prime}\right\|_{0}\left\|\Psi^{\prime-1} \Psi\right\|_{0} \leq \epsilon_{j}
$$

so it is possible to iterate Proposition 2.13.

Conclusion After $l-1$ steps,

$$
\left|\Psi^{-1} \Psi^{\prime} F_{l+1} \Psi^{\prime-1} \Psi\right|_{r_{l}} \leq \epsilon^{\prime \frac{17}{16}}
$$

Let $\left.Z=e^{X_{2}} \ldots e^{X_{l+1}} \in C_{r^{\prime}}^{\omega}\left(2 \mathbb{T}^{d}, G\right)\right), A^{\prime}=A_{l+1}, F^{\prime}=F_{l+1}$. Then

$$
\partial_{\omega} Z=\left(A_{1}+F_{1}\right) Z-Z\left(A^{\prime}+F^{\prime}\right)
$$

and

$$
\left\|A^{\prime}\right\| \leq\left\|A_{1}\right\|+\sum_{j=1}^{l}\left\|\hat{F}_{j}(0)\right\|+4 \pi \bar{N} \leq\|A\|+|\log \epsilon|\left(\frac{1}{r-r^{\prime}}\right)^{D_{4}}
$$

for $D_{4}$ great enough depending only on $n$, whence property 5 , If $\mathcal{G}=\operatorname{sl}(2, \mathbb{C})$ or $\operatorname{sl}(2, \mathbb{R})$ and $\Psi^{\prime-1} \Psi$ is not the identity, then 


$$
\left\|A^{\prime}\right\| \leq\left\|A_{1}\right\|+\sum_{j=1}^{l}\left\|\hat{F}_{j}(0)\right\| \leq \kappa^{\prime \prime}\left(r, r^{\prime \prime}, \epsilon\right)+\epsilon^{\frac{1}{2}}
$$

To prove that $\mathcal{L}_{l+1}$ is indeed an $\left(A_{l+1}, \kappa^{\prime \prime}\left(r^{\prime \prime}, r-\frac{r-r^{\prime \prime}}{2}, \epsilon^{\prime}\right), 2 \gamma\right)$-decomposition, it is enough to show that

$$
\kappa^{\prime \prime}\left(r^{\prime \prime}, r^{\prime \prime}-\frac{r-r^{\prime \prime}}{2}, \epsilon^{\prime}\right) \leq\left(\frac{3}{4}\right)^{l+1} \frac{\kappa^{\prime \prime}}{C_{0}}
$$

which comes from the definition of the function $\kappa^{\prime \prime}$.

Let us prove property 4. It is true that

$$
\left|\Psi^{\prime}\right|_{r^{\prime \prime}} \leq \epsilon^{-\frac{1}{2}\left(r-r^{\prime \prime}\right)} \epsilon^{-\frac{1}{96}} e^{4 \pi r \bar{N}} \leq \epsilon^{-\frac{1}{2}\left(r-r^{\prime \prime}\right)} \epsilon^{-\frac{1}{96}} \epsilon^{-\frac{r-r^{\prime \prime}}{200}}
$$

and property 4 comes from it, since

$$
\epsilon=\epsilon^{\frac{|\log \epsilon|}{2 \pi \sqrt[4]{R} \bar{N}\left(r-r^{\prime \prime}\right)}}
$$

Moreover,

$$
\left|\Psi^{\prime} F^{\prime} \Psi^{\prime-1}\right|_{r^{\prime \prime}} \leq|\Psi|_{r}\left|\Psi^{-1}\right|_{r}\left|\Psi^{-1} \Psi^{\prime} F^{\prime} \Psi^{\prime-1} \Psi\right|_{r^{\prime \prime}} \leq \epsilon^{\prime}
$$

whence 3, Let $Z^{\prime}=Z_{1} \Psi^{\prime} Z \Psi^{\prime-1}, \bar{F}^{\prime}=\Psi^{\prime} F^{\prime} \Psi^{-1}$ (which satisfies property 2) and $\bar{A}$ such that

$$
\partial_{\omega} \Psi^{\prime}=\bar{A}^{\prime} \Psi^{\prime}-\Psi^{\prime} A^{\prime}
$$

Then 6 and 1 hold, and by (87),

$$
\begin{aligned}
\left|Z^{\prime}-I d\right|_{r^{\prime \prime}} & \leq\left|Z_{1}-I d\right|_{r_{1}}+|\Psi|_{r}\left|\Psi^{-1}\right|_{r} \sum_{j}\left|\Psi^{-1} \Psi^{\prime} X_{j} \Psi^{\prime-1} \Psi\right|_{r_{j}} \\
& \leq \frac{1}{\tilde{C}^{\prime}}\left(\frac{l\left(1+|| A_{\mathcal{N}}||\right)|\log \epsilon|}{r-r^{\prime \prime}}\right)^{D_{1} \gamma}\left(\frac{1}{\epsilon}\right)^{4\left(r-r^{\prime \prime}\right)}\left(\epsilon+\sum_{j}\left|\Psi^{-1} \Psi^{\prime} F_{j} \Psi^{\prime-1} \Psi\right|_{r_{j}}\right)
\end{aligned}
$$

and by (88) and (96),

$$
\left|Z^{\prime}-I d\right|_{r^{\prime \prime}} \leq \frac{2}{\tilde{C}^{\prime}}\left(\frac{l\left(1+|| A_{\mathcal{N}}||\right)|\log \epsilon|}{r-r^{\prime \prime}}\right)^{D_{1} \gamma}\left(\frac{1}{\epsilon}\right)^{4\left(r-r^{\prime \prime}\right)} \epsilon
$$

whence property 7 with $D_{3} \gamma \geq 2 D_{1} \gamma$ if $C^{\prime} \leq \frac{\tilde{C}^{\prime}}{2\left(l\left(r-r^{\prime \prime}\right)\right)^{D_{1} \gamma}}$, since $l\left(r-r^{\prime \prime}\right)$ has a bound which is independent of $r-r^{\prime \prime}$.

This proposition is the inductive step which can be iterated as a whole. It is necessary to obtain an $\epsilon^{\prime}$ which is much smaller than $\epsilon$ so as to control $\left|\Psi^{\prime}\right|_{r^{\prime}}$ as a function of $\epsilon^{\prime}$ and make sure that the output be similar to the input. 


\subsection{Main theorem}

First let us give a lemma which will enable us to iterate Proposition 2.14.

Lemma 2.15 Let $C^{\prime} \leq 1, b_{0}>0, r \leq \frac{1}{2}$ and $r^{\prime} \in\left[\frac{95}{96} r, r\left[\right.\right.$. Let $D_{5}, \gamma_{0} \in \mathbb{N}$. There exists $C$ depending only on $C^{\prime}, D_{5}, \gamma_{0}$ such that for all $\epsilon \leq C\left(\frac{r-r^{\prime}}{b_{0}+1}\right)^{2 \gamma_{0} D_{5}}$, choosing a sequence $\left(\epsilon_{k}\right)$ such that for all $k$,

$$
\epsilon_{k} \leq \epsilon_{k-1}^{100}<1
$$

and letting for all $k$

$$
\left\{\begin{array}{c}
\gamma_{k}=2^{k} \gamma_{0} \\
r_{k}=r^{\prime}+\frac{r-r^{\prime}}{2^{k}} \\
b_{k}=b_{k-1}+\left|\log \epsilon_{k-1}\right|\left(\frac{2^{k}}{r-r^{\prime}}\right)^{D_{5}}
\end{array}\right.
$$

then for every $k \in \mathbb{N}$,

$$
\left|\log \epsilon_{k}\right|^{2 D_{5} \gamma_{k}} \leq \epsilon_{k}^{-\frac{1}{4}}
$$

and

$$
\left(\frac{b_{k}+1}{r_{k}-r_{k+1}}\right)^{D_{5} \gamma_{k}} \epsilon_{k} \leq C^{\prime}
$$

Proof: Let us first prove (98). It is equivalent to

$$
2^{k+3} D_{5} \gamma_{0} \leq \frac{\left|\log \epsilon_{k}\right|}{\log \left|\log \epsilon_{k}\right|}
$$

The function $t \mapsto \frac{|\log t|}{\log |\log t|}$ is decreasing for $\left.\left.t \in\right] 0, e^{-\frac{1}{e}}\right]$ so it is enough to show that

$$
2^{k+3} D_{5} \gamma_{0} \leq \frac{100^{k}|\log \epsilon|}{k \log 100+\log |\log \epsilon|}
$$

which is true if we choose $C$ as a function of $D_{5}, \gamma_{0}$.

- Let $a_{k}=\left(\frac{b_{k}+1}{r_{k}-r_{k+1}}\right)^{D_{5} \gamma_{k}} \epsilon_{k}$. For all $k$,

$$
\begin{aligned}
a_{k+1} & =\left(\frac{\left(b_{k+1}+1\right) 2^{k+2}}{r-r^{\prime}}\right)^{D_{5} \gamma_{k+1}} \epsilon_{k+1} \\
& \leq\left(\frac{\left(b_{0}+(k+1)\left|\log \epsilon_{k}\right|\right) 2^{k+2}}{r-r^{\prime}}\right)^{2 D_{5} \gamma_{k+1}} \frac{\epsilon_{k+1}}{\epsilon_{k}} a_{k}
\end{aligned}
$$

so by (98),

$$
a_{k+1} \leq\left(\frac{\left(b_{0}+1\right)}{r-r^{\prime}}\right)^{\gamma_{0} 16^{k+1} D_{5}} \epsilon^{100^{k} .98} a_{k}
$$


thus, if $\epsilon$ is also smaller than $\left(\frac{r-r^{\prime}}{b_{0}+1}\right)^{16 \gamma_{0} D_{5}}$, then $a_{k+1} \leq a_{k}$. If $\epsilon$ is also small enough to satisfy

$$
a_{0}=\left(\frac{b_{0}+1}{r-r^{\prime}}\right)^{D_{5} \gamma_{0}} \epsilon \leq C^{\prime}
$$

for instance

$$
\left(\frac{b_{0}+1}{r-r^{\prime}}\right)^{D_{5} \gamma_{0}} \epsilon^{\frac{3}{4}} \leq C^{\prime}
$$

then (99) is true for all $k$.

Lemma 2.15 implies that assumption (80) of Proposition 2.14 holds for all $k$ with $\epsilon \leq \epsilon_{k}$, $\|A\|=b_{k}, r=r_{k}$ and $r^{\prime \prime}=r_{k+1}$.

As a consequence, one gets the main result, of which we will give various formulations.

Theorem 2.16 Let $r \leq \frac{1}{2}, A \in \mathcal{G}$ and $F \in C_{r}^{\omega}\left(2 \mathbb{T}^{d}, \mathcal{G}\right)$ with nice periodicity properties with respect to $\mathcal{L}_{A}$. Let

$$
r^{\prime} \in\left[\frac{95}{96} r, r[\right.
$$

There exists $D_{7}$ depending only on $n, d, \tau, \kappa, A$ such that if

$$
|F|_{r} \leq \epsilon_{0}^{\prime}\left(r, r^{\prime}\right)=\left(\frac{r-r^{\prime}}{\|A\|+1}\right)^{D_{7}}
$$

then for any $\epsilon \leq \epsilon_{0}^{\prime}$, there exists

- $Z_{\epsilon}, \Psi_{\epsilon} \in C_{r^{\prime}}^{\omega}\left(2 \mathbb{T}^{d}, G\right)$,

- $A_{\epsilon} \in \mathcal{G}$,

- $\bar{A}_{\epsilon}, \bar{F}_{\epsilon} \in C_{r^{\prime}}^{\omega}\left(2 \mathbb{T}^{d}, \mathcal{G}\right)$,

such that

1. $\bar{A}_{\epsilon}$ is reducible to $A_{\epsilon}$ by $\Psi_{\epsilon}$,

2. $\left|\bar{F}_{\epsilon}\right|_{r^{\prime}} \leq \epsilon$

3. for every $\theta \in 2 \mathbb{T}^{d}$,

$$
\partial_{\omega} Z_{\epsilon}(\theta)=(A+F(\theta)) Z_{\epsilon}(\theta)-Z_{\epsilon}(\theta)\left(\bar{A}_{\epsilon}(\theta)+\bar{F}_{\epsilon}(\theta)\right)
$$

4.

$$
\left|Z_{\epsilon}-I d\right|_{r^{\prime}} \leq 2^{D_{7}} \epsilon_{0}^{\frac{1}{4}-4\left(r-r^{\prime}\right)}
$$

and so does $Z_{\epsilon}^{-1}-I d$, 
5. $Z_{\epsilon}, \partial_{\omega} Z_{\epsilon}$ are bounded in $C_{r^{\prime}}^{\omega}\left(2 \mathbb{T}^{d}, g l(n, \mathbb{C})\right)$ uniformly in $\epsilon$,

6 .

$$
\left|\Psi_{\epsilon}\right|_{r^{\prime}} \leq \epsilon^{-\left(\frac{1}{2}\right)^{c^{\prime} \sqrt{\log |\log \epsilon|}}}
$$

where $c^{\prime}$ only depends on $n, d, \kappa, \tau, A$.

Moreover,

- in dimension 2 or if $\mathcal{G}=\operatorname{gl}(n, \mathbb{C})$ or $u(n)$, if $F$ is continuous on $\mathbb{T}^{d}$, then $\bar{A}_{\epsilon}, \bar{F}_{\epsilon}$ and $Z_{\epsilon}$ are continuous on $\mathbb{T}^{d}$.

- If $\mathcal{G}$ is o(n) or $u(n)$, then $D_{7}$ does not depend on $A$ and the same holds replacing (100) by $|F|_{r} \leq\left(r-r^{\prime}\right)^{D_{7}}$.

- if $\mathcal{G}=\operatorname{sl}(2, \mathbb{C})$ or $\operatorname{sl}(2, \mathbb{R})$ and $A+F$ is not reducible, then there exists a sequence $\epsilon_{k} \rightarrow 0$ such that $\left\|A_{\epsilon_{k}}\right\|\left|\log \epsilon_{k}\right|^{\tau}$ is bounded.

Proof: The proof will be made by induction as follows. Let $r^{\prime \prime}=\frac{r+r^{\prime}}{2}$. Let $R\left(r, r^{\prime \prime}\right), N(r, \epsilon)$, $\kappa^{\prime \prime}\left(r, r^{\prime \prime}, \epsilon\right)$ be as in (79). There exists $\gamma_{0} \in \mathbb{N}$ depending only on $n, d, \tau, \kappa, A$, such that $\mathcal{L}_{A}$ is an $\left(A, \kappa, \gamma_{0}\right)$-decomposition (one can assume $\gamma_{0} \geq n(n+1)$ ). Let $C^{\prime}, D_{3}$ be as in Proposition 2.14. Let $D_{5}=2 D_{3}$. Let $C$ be as in Lemma 2.15 and $D_{7}$ such that

$$
\left(\frac{r-r^{\prime \prime}}{\|A\|+1}\right)^{D_{7}} \leq C\left(\frac{r-r^{\prime \prime}}{\|A\|+1}\right)^{4 \gamma_{0} D_{5}}
$$

Let

$$
\epsilon_{0}^{\prime}=\left(\frac{r-r^{\prime \prime}}{\|A\|+1}\right)^{D_{7}}
$$

Before carrying on with the proof, note that if $\mathcal{G}$ is $o(n)$ or $u(n)$, then $\mathcal{L}_{A}$ is a unitary decomposition, therefore it is an $(A, \kappa, 0)$-decomposition, so one can take $\gamma_{0}=n(n+1)$ and then $\gamma_{0}, D_{3}, D_{5}$ and $D_{7}$ do not depend on $A$. For all $k \in \mathbb{N}$, let

$$
\left\{\begin{array}{c}
r_{k}=r^{\prime \prime}+\frac{r-r^{\prime \prime}}{2^{k}} \\
b_{0}=\|A\|, \\
b_{k+1}=\|A\|+\sum_{j \leq k} \frac{\left|\log \epsilon_{j}\right|}{\left(r_{j-1}-r_{j}\right)^{D_{5}}}
\end{array}\right.
$$

where $\left(\epsilon_{j}\right)$ will be defined by induction in the following. Suppose that $|F|_{r} \leq \epsilon_{0}^{\prime}$. Let $\bar{F}_{1}=F, \bar{A}_{1}=A_{1}=A$ and $\Psi_{0}=I d$. Iterate Proposition 2.14 using lemma 2.15 to find, for all $k \geq 1$,

- $Z_{k+1} \in C_{r_{k+1}}^{\omega}\left(2 \mathbb{T}^{d}, G\right)$,

- $A_{k+1} \in \mathcal{G}$,

- $\bar{A}_{k+1} \in C_{r}^{\omega}\left(2 \mathbb{T}^{d}, \mathcal{G}\right)$,

- $\Psi_{k} \in C_{r}^{\omega}\left(2 \mathbb{T}^{d}, G\right)$, 
- $\bar{F}_{k+1} \in C_{r_{k+1}}^{\omega}\left(2 \mathbb{T}^{d}, \mathcal{G}\right)$

- $\epsilon_{k+1} \in\left[\epsilon_{k}^{R\left(r_{k}, r_{k+1}\right)^{n^{2}}}, \epsilon_{k}^{100}\right]$

such that

1. $\bar{A}_{k+1}$ is reducible to $A_{k+1}$ by $\Psi_{k}$,

2. $\Psi_{k}^{-1} \bar{F}_{k+1} \Psi_{k}$ has nice periodicity properties with respect to an $\left(A_{k+1}, \kappa^{\prime \prime}\left(r_{k+1}, r_{k+2}, \epsilon_{k+1}\right), 2^{k+1} \gamma_{0}\right)$ decomposition,

3. $\left|\bar{F}_{k+1}\right|_{r_{k+1}} \leq \epsilon_{k+1}$,

4. $\left|\Psi_{k}\right|_{r} \leq \epsilon_{k+1}^{-\frac{1}{2}\left(r_{k+1}-r_{k+2}\right)}$ and $\left|\Psi_{k}^{-1}\right|_{r} \leq \epsilon_{k+1}^{-\frac{1}{2}\left(r_{k+1}-r_{k+2}\right)}$,

5. $\left\|A_{k+1}\right\| \leq b_{k+1}$, and, if $\mathcal{G}=\operatorname{sl}(2, \mathbb{C})$ or $\operatorname{sl}(2, \mathbb{R})$ and $\Psi_{k}^{-1} \Psi_{k-1}$ is not the identity, $\left\|A_{k+1}\right\| \leq \kappa^{\prime \prime}\left(r_{k}, r_{k+1}, \epsilon_{k}\right)+\epsilon_{k}^{\frac{1}{2}}$

6.

$$
\partial_{\omega} Z_{k+1}=\left(\bar{A}_{k}+\bar{F}_{k}\right) Z_{k+1}-Z_{k+1}\left(\bar{A}_{k+1}+\bar{F}_{k+1}\right)
$$

7.

$$
\left|Z_{k+1}-I d\right|_{r_{k+1}} \leq \frac{1}{C^{\prime}}\left(\frac{\left(1+|| A_{k}||\right)\left|\log \epsilon_{k}\right|}{r_{k}-r_{k+1}}\right)^{2^{k} D_{3} \gamma_{0}} \epsilon_{k}^{1-4\left(r_{k}-r_{k+1}\right)}
$$

which implies, using Lemma 2.15, that

$$
\left|Z_{k+1}-I d\right|_{r_{k+1}} \leq \frac{1}{C^{\prime}} \epsilon_{k}^{\frac{1}{4}-4\left(r_{k}-r_{k+1}\right)}
$$

and so does $Z_{k+1}^{-1}-I d$.

- Let $\epsilon \leq \epsilon_{0}^{\prime}$ and $k_{\epsilon} \in \mathbb{N}$ such that $\epsilon_{k_{\epsilon}+1} \leq \epsilon \leq \epsilon_{k_{\epsilon}}$. Let

$$
\left\{\begin{array}{c}
Z_{\epsilon}=Z_{1} \ldots Z_{k_{\epsilon}} \\
\bar{A}_{\epsilon}=\bar{A}_{k_{\epsilon}} \\
\bar{F}_{\epsilon}=\bar{F}_{k_{\epsilon}}
\end{array}\right.
$$

then properties 1 and 2 hold. Thus for all $\theta \in 2 \mathbb{T}^{d}$,

$$
\partial_{\omega} Z_{\epsilon}(\theta)=(A+F(\theta)) Z_{\epsilon}(\theta)-Z_{\epsilon}(\theta)\left(\bar{A}_{\epsilon}(\theta)+\bar{F}_{\epsilon}(\theta)\right)
$$

whence property 3, Moreover, let $a_{k}:=\left|Z_{1} \ldots Z_{k}-I d\right|_{r^{\prime \prime}}$, then 


$$
a_{1}=\left|Z_{1}-I d\right|_{r^{\prime \prime}} \leq \frac{1}{C^{\prime}} \epsilon_{0}^{\frac{1}{4}-4\left(r_{0}-r_{1}\right)}
$$

so

$$
\left|Z_{1}\right|_{r^{\prime \prime}} \leq 1+\frac{1}{C^{\prime}} \epsilon_{0}^{\frac{1}{4}-4\left(r_{0}-r_{1}\right)}
$$

- let $k \geq 2$ and assume that for all $j \leq k-1$,

$$
\left|Z_{1} \ldots Z_{j}\right|_{r^{\prime \prime}} \leq 1+\frac{3}{C^{\prime}} \epsilon_{0}^{\frac{1}{4}-4\left(r_{0}-r_{1}\right)}
$$

then

$$
\begin{aligned}
a_{k} & \leq\left|Z_{k}-I d\right|_{r^{\prime \prime}}\left|Z_{1} \ldots Z_{k-1}\right|_{r^{\prime \prime}}+a_{k-1} \\
& \leq a_{1}+\frac{1}{C^{\prime}} \sum_{j=1}^{k-1}\left|Z_{1} \ldots Z_{j}\right|_{r^{\prime \prime}} \epsilon_{j}^{\frac{1}{4}-4\left(r_{j}-r_{j+1}\right)} \leq \frac{3}{C^{\prime}} \epsilon_{0}^{\frac{1}{4}-4\left(r_{0}-r_{1}\right)}
\end{aligned}
$$

and

$$
\left|Z_{1} \ldots Z_{k}\right|_{r^{\prime \prime}} \leq 1+\frac{3}{C^{\prime}} \epsilon_{0}^{\frac{1}{4}-4\left(r_{0}-r_{1}\right)}
$$

whence property 4. This also implies that

$$
\left|Z_{\epsilon}\right|_{r^{\prime \prime}} \leq 2+\frac{3}{C^{\prime}} \epsilon_{0}^{\frac{1}{4}-4\left(r_{0}-r_{1}\right)}
$$

Moreover, by a Cauchy estimate,

$$
\left|\partial_{\omega} Z_{\epsilon}\right|_{r^{\prime}} \leq \frac{1}{r^{\prime \prime}-r^{\prime}}\left|Z_{\epsilon}\right|_{r^{\prime \prime}}
$$

so 5 is true. Also note that

$$
\left|\Psi_{\epsilon}\right|_{r^{\prime \prime}}=\left|\Psi_{k_{\epsilon}-1}\right|_{r^{\prime \prime}} \leq \epsilon_{k_{\epsilon}}^{-\frac{1}{2}\left(r_{k_{\epsilon}}-r_{k_{\epsilon}+1}\right)} \leq \epsilon^{-\frac{1}{2^{k_{\epsilon}+2}}}
$$

Since

$$
k_{\epsilon}\left(k_{\epsilon}+1\right) \geq c \log \left(\frac{|\log \epsilon|}{\left|\log \left(|F|_{r}\right)\right|}\right)
$$

where $c$ only depends on $n, d, \kappa, \tau, A$, then

$$
\left|\Psi_{\epsilon}\right|_{r^{\prime \prime}} \leq \epsilon^{-\left(\frac{1}{2}\right)^{c^{\prime} \sqrt{\log |\log \epsilon|}}}
$$

where $c^{\prime}$ only depends on $n, d, \kappa, \tau, A$; therefore property $[6$ holds. 
If $\mathcal{G}$ is either $g l(n, \mathbb{C})$ or $u(n)$ or in dimension 2 , if $F$ is continuous on $\mathbb{T}^{d}$, each step will give functions $Z_{k+1}, A_{k+1}, \bar{A}_{k+1}, \bar{F}_{k+1}$ continuous on $\mathbb{T}^{d}$ so, at the end of the process, the functions $Z_{\epsilon}, \bar{A}_{\epsilon}$ et $\bar{F}_{\epsilon}$ are continuous on $\mathbb{T}^{d}$. The fact that $\left\|A_{\epsilon_{k}}\right\|\left|\log \epsilon_{k}\right|^{\tau}$ is bounded for some sequence $\epsilon_{k}$ if $\mathcal{G}=\operatorname{sl}(2, \mathbb{C})$ or $\operatorname{sl}(2, \mathbb{R})$ and $A+F$ is not reducible comes from property 5 in the iteration.

This proves Theorem 1.1.

In general, almost reducibility does not imply reducibility. Reducibility happens if there are a finite number of steps at which one has to reduce the eigenvalues, or if the sequence $\left(\Psi_{k}\right)$ given by Theorem 2.16 converges in $C_{r^{\prime}}^{\omega}\left(2 \mathbb{T}^{d}, G\right)$. In general, this sequence is not even bounded in $C_{0}^{\omega}\left(2 \mathbb{T}^{d}, G\right)$. However, if the method above has been used to conjugate the system $A+F$ to a system $\bar{A}_{\epsilon}+\bar{F}_{\epsilon}$ where $\bar{A}_{\epsilon}$ is reducible by $\Psi_{\epsilon}$ to a constant $A_{\epsilon}$, and where $\bar{F}_{\epsilon}$ is bounded by $\epsilon$, one can also bound $\Psi_{\epsilon}^{-1} \bar{F}_{\epsilon} \Psi_{\epsilon}$.

Corollary 2.17 Let $r \leq \frac{1}{2}, A \in \mathcal{G}$ and $F \in C_{r}^{\omega}\left(2 \mathbb{T}^{d}, \mathcal{G}\right)$ with nice periodicity properties with respect to $\mathcal{L}_{A}$. Let $r^{\prime} \in\left[\frac{95}{96} r, r\left[\right.\right.$. There exists $D_{8}$ depending only on $n, d, \kappa, \tau, A$ such that if

$$
|F|_{r} \leq\left(r-r^{\prime}\right)^{D_{8}}
$$

then there exists

- $Z \in C_{r^{\prime}}^{\omega}\left(2 \mathbb{T}^{d}, G\right)$,

- a family $\left(A_{l}\right)$ of reducible functions in $C_{r^{\prime}}^{\omega}\left(2 \mathbb{T}^{d}, \mathcal{G}\right)$

- and $A_{\infty} \in C_{r^{\prime}}^{\omega}\left(2 \mathbb{T}^{d}, \mathcal{G}\right)$

such that

$$
\partial_{\omega} Z(\theta)=(A+F(\theta)) Z(\theta)-Z(\theta) A_{\infty}(\theta)
$$

and

$$
\lim _{l \rightarrow \infty}\left|A_{l}-A_{\infty}\right|_{r^{\prime}}=0
$$

Moreover, in dimension 2 or if $\mathcal{G}=g l(n, \mathbb{C})$ or $u(n)$, if $F$ is continuous on $\mathbb{T}^{d}$, then $Z$, $A_{l}$ and $A_{\infty}$ are continuous on $\mathbb{T}^{d}$. Finally, if $\mathcal{G}=o(n)$ or $u(n)$, then $D_{8}$ does not depend on $A$.

Proof: Let $D_{7}$ be as in Theorem 2.16 and $D_{8}$ such that

$$
\left(r-r^{\prime}\right)^{D_{8}} \leq\left(\frac{r-r^{\prime}}{1+\|A\|}\right)^{D_{7}}
$$

Let $Z_{\epsilon} \in C_{r^{\prime}}^{\omega}\left(2 \mathbb{T}^{d}, G\right), A_{\epsilon} \in C_{r^{\prime}}^{\omega}\left(2 \mathbb{T}^{d}, \mathcal{G}\right)$ be as in Theorem 2.16. Then $Z_{\epsilon}$ and $\partial_{\omega} Z_{\epsilon}$ remain bounded in $C_{r^{\prime}}^{\omega}\left(2 \mathbb{T}^{d}, G\right)$ when $\epsilon \rightarrow 0$. Let $Z$ be the limit in $C_{r^{\prime}}^{\omega}\left(2 \mathbb{T}^{d}, G\right)$ of a subsequence $\left(Z_{\frac{1}{k_{l}}}\right)$ of $\left(Z_{\frac{1}{k}}\right)_{k \in \mathbb{N} \backslash\{0\}}$ and 


$$
A_{\infty}(\theta):=Z(\theta)^{-1}(A+F(\theta)) Z(\theta)-Z(\theta)^{-1} \partial_{\omega} Z(\theta)
$$

then

$$
A_{\infty} \in C_{r^{\prime}}^{\omega}\left(2 \mathbb{T}^{d}, \mathcal{G}\right), \lim _{l \rightarrow \infty}\left|A_{\frac{1}{k_{l}}}-A_{\infty}\right|_{r^{\prime}}=0
$$

and so equation (103) holds.

In dimension 2 or if $\mathcal{G}=g l(n, \mathbb{C})$ or $u(n)$, if $F$ is continuous on $\mathbb{T}^{d}$, all functions that one has to consider are continuous on $\mathbb{T}^{d}$.

Remark: In Corollary 2.17, the function $A_{\infty}$ is not reducible in general, it is only a limit of reducible functions.

Corollary 2.18 Let $0<r^{\prime}<r \leq \frac{1}{2}, A \in \mathcal{G}$ and $F \in C_{r}^{\omega}\left(\mathbb{T}^{d}, \mathcal{G}\right)$. There exists $\epsilon_{0}^{\prime}$ depending only on $n, d, \tau, \kappa, A, r-r^{\prime}$ such that if $|F-A|_{r} \leq \epsilon_{0}^{\prime}$, then for all $\epsilon>0$ there exists $H \in C_{r^{\prime}}^{\omega}\left(2 \mathbb{T}^{d}, \mathcal{G}\right)$ such that $|F-H|_{r^{\prime}} \leq \epsilon$ and $H$ is reducible.

Proof: Let $D_{7}$ be as in Theorem 2.16. Assume that

$$
|F-A|_{r} \leq\left(r-r^{\prime}\right)^{D_{7}}=: \epsilon_{0}^{\prime}
$$

Let $\epsilon>0$. By Theorem 2.16, there exist $Z_{\epsilon} \in C_{r^{\prime}}^{\omega}\left(2 \mathbb{T}^{d}, G\right), \bar{A}_{\epsilon}, \bar{F}_{\epsilon} \in C_{r^{\prime}}^{\omega}\left(2 \mathbb{T}^{d}, \mathcal{G}\right)$ and $A_{\epsilon} \in \mathcal{G}$ such that

- $\bar{A}_{\epsilon}$ is reducible to $A_{\epsilon}$,

- $\partial_{\omega} Z_{\epsilon}=F Z_{\epsilon}-Z_{\epsilon}\left(\bar{A}_{\epsilon}+\bar{F}_{\epsilon}\right)$,

- $\left|Z_{\epsilon}\right|_{r^{\prime}} \leq 2,\left|Z_{\epsilon}^{-1}\right|_{r^{\prime}} \leq 2$,

- $\left|\bar{F}_{\epsilon}\right|_{r^{\prime}} \leq \frac{\epsilon}{4}$.

Therefore

$$
\partial_{\omega} Z_{\epsilon}=H Z_{\epsilon}-Z_{\epsilon} \bar{A}_{\epsilon}
$$

where $H=F-Z_{\epsilon} \bar{F}_{\epsilon} Z_{\epsilon}^{-1}$ is reducible to $A_{\epsilon}$ and satisfies

$$
|H-F|_{r^{\prime}} \leq 4\left|\bar{F}_{\epsilon}\right|_{r^{\prime}} \leq \epsilon
$$

Corollary 2.19 Let $0<r^{\prime}<r \leq \frac{1}{2}, A \in \operatorname{sl}(2, \mathbb{R})$ and $F \in C_{r}^{\omega}\left(\mathbb{T}^{d}\right.$, sl $\left.(2, \mathbb{R})\right)$. There exists $\epsilon_{0}^{\prime}$ depending only on $n, d, \tau, \kappa, A, r-r^{\prime}$ such that if $|F-A|_{r} \leq \epsilon_{0}^{\prime}$, then for any $\epsilon>0$ there exist $H \in C_{r^{\prime}}^{\omega}\left(\mathbb{T}^{d}\right.$, sl $\left.(2, \mathbb{R})\right)$ such that $|F-H|_{r^{\prime}} \leq \epsilon$ and $H$ is reducible.

Proof: Do the same construction as in Corollary 2.18, Theorem 2.16 gives functions $\bar{A}_{\epsilon}, \bar{F}_{\epsilon}, Z_{\epsilon}$ which are, in fact, continuous on $\mathbb{T}^{d}$. Thus $H$ is continuous on $\mathbb{T}^{d}$.

Corollary 2.19 also holds with $g l(n, \mathbb{C})$ or $u(n)$ instead of $\operatorname{sl}(2, \mathbb{R})$. This proves Theorem 1.3. Again, note that if $G$ is a compact group, then the smallness condition does not depend on $A$. 


\section{Strong almost reducibility for quasi-periodic cocycles in a Gevrey class}

Let $\beta>1$ and $r>0$. Let $C_{r}^{G, \beta}\left(2 \mathbb{T}^{d}, g l(n, \mathbb{C})\right)$ be the functions of class Gevrey $\beta$ with parameter $r$, i.e the functions $F \in C^{\infty}\left(2 \mathbb{T}^{d}, g l(n, \mathbb{C})\right)$ satisfying

$$
\sum_{\alpha \in \mathbb{N}^{d}} \frac{r^{\beta|\alpha|}}{\alpha !^{\beta}} \sup _{\theta}\left\|\partial^{\alpha} F(\theta)\right\|<+\infty
$$

Denote by $\|\cdot\|_{\beta, r}$ the norm

$$
\|F\|_{\beta, r}=\sum_{\alpha \in \mathbb{N}^{d}} \frac{r^{\beta|\alpha|}}{\alpha !^{\beta}} \sup _{\theta}\left\|\partial^{\alpha} F(\theta)\right\|
$$

The main theorem in this part is formulated analogously to Theorem 1.1.

Theorem 3.1 Let $0<r^{\prime}<r \leq \frac{1}{2}, A \in \mathcal{G}, F \in C_{r}^{G, \beta}\left(\mathbb{T}^{d}, \mathcal{G}\right)$. There is $\epsilon_{0}<1$ depending only on $n, d, \kappa, \tau, A, r-r^{\prime}$ such that if

$$
\|F\|_{\beta, r} \leq \epsilon_{0}
$$

then for all $\epsilon>0$, there exists $\bar{A}_{\epsilon}, \bar{F}_{\epsilon} \in C_{r^{\prime}}^{G, \beta}\left(2 \mathbb{T}^{d}, \mathcal{G}\right), \Psi_{\epsilon}, Z_{\epsilon} \in C_{r^{\prime}}^{G, \beta}\left(2 \mathbb{T}^{d}, G\right)$ and $A_{\epsilon} \in \mathcal{G}$ such that for all $\theta \in 2 \mathbb{T}^{d}$,

$$
\partial_{\omega} Z_{\epsilon}(\theta)=(A+F(\theta)) Z_{\epsilon}(\theta)-Z_{\epsilon}(\theta)\left(\bar{A}_{\epsilon}(\theta)+\bar{F}_{\epsilon}(\theta)\right)
$$

with

- $\bar{A}_{\epsilon}$ reducible to $A_{\epsilon}$ by $\Psi_{\epsilon}$,

- $\left\|\bar{F}_{\epsilon}\right\|_{\beta, r^{\prime}} \leq \epsilon$,

- $\left\|\Psi_{\epsilon}\right\|_{\beta, r^{\prime}} \leq \epsilon^{-\frac{1}{8}}$,

- and $\left\|Z_{\epsilon}-I d\right\|_{\beta, r^{\prime}} \leq 2 \epsilon_{0}^{\frac{1}{2}}$.

Moreover,

- in dimension 2 or if $G=G L(n, \mathbb{C})$ or $U(n), Z_{\epsilon}, \bar{A}_{\epsilon}, \bar{F}_{\epsilon}$ are continuous on $\mathbb{T}^{d}$;

- If $\mathcal{G}$ is o(n) or $u(n)$, then $\epsilon_{0}$ does not depend on $A$;

- if $\mathcal{G}=\operatorname{sl}(2, \mathbb{C})$ or $\operatorname{sl}(2, \mathbb{R})$ and $A+F$ is not reducible, then there exists a sequence $\epsilon_{k} \rightarrow 0$ such that $\left\|A_{\epsilon_{k}}\right\|\left|\log \epsilon_{k}\right|^{\tau}$ is bounded. 


\subsection{Preliminaries on Gevrey class functions}

\section{Remark:}

- For all $0<r^{\prime}<r$, one has the inclusion $C_{r}^{G, \beta}\left(2 \mathbb{T}^{d}, \mathcal{G}\right) \subset C_{r^{\prime}}^{G, \beta}\left(2 \mathbb{T}^{d}, \mathcal{G}\right)$ and

$$
\|f\|_{\beta, r^{\prime}} \leq\|f\|_{\beta, r}
$$

- For $f, g \in C_{r}^{G, \beta}\left(2 \mathbb{T}^{d}, \mathcal{G}\right)$, one has $\|f g\|_{\beta, r} \leq\|f\|_{\beta, r}\|g\|_{\beta, r}$ (see [8], appendix).

Lemma 3.2 For all $m \in \mathbb{Z}^{d}$ and all $r^{\prime}>0$, the map $\theta \mapsto e^{2 i \pi\langle m, \theta\rangle}$ satisfies

$$
\|\left. e^{2 i \pi\langle m, \cdot\rangle}\right|_{\beta, r^{\prime}} \leq e^{\beta \pi r^{\prime} d|m|^{\frac{1}{\beta}}}
$$

Proof: For all $\alpha \in \mathbb{N}^{d}$ and all $\theta \in \mathbb{T}^{d}$,

$$
\begin{aligned}
\frac{r^{\prime \beta|\alpha|}}{(\alpha !)^{\beta}}\left|\partial^{\alpha}\left(e^{2 i \pi\langle m, \theta\rangle}\right)\right| & \leq \frac{r^{\prime \beta|\alpha|}}{(\alpha !)^{\beta}} \prod_{j}\left|2 \pi m_{j}\right|^{\alpha_{j}} \\
& \leq \prod_{j} \frac{\left(r^{\prime \beta}\left|2 \pi m_{j}\right|\right)^{\alpha_{j}}}{\left(\alpha_{j} !\right)^{\beta}} \\
& \leq \prod_{j}\left(\frac{\left(r^{\prime}\left|2 \pi m_{j}\right|^{\frac{1}{\beta}}\right)^{\alpha_{j}}}{\alpha_{j} !}\right)^{\beta}
\end{aligned}
$$

thus

$$
\begin{aligned}
\sum_{\alpha} \frac{r^{\prime \beta|\alpha|}}{(\alpha !)^{\beta}}\left|\partial^{\alpha}\left(e^{2 i \pi\langle m, \theta\rangle}\right)\right| & \leq \prod_{j} \sum_{\alpha_{j}}\left(\frac{\left(r^{\prime}\left|2 \pi m_{j}\right|^{\frac{1}{\beta}}\right)^{\alpha_{j}}}{\alpha_{j} !}\right)^{\beta} \\
& \leq \prod_{j}\left(\sum_{\alpha_{j}} \frac{\left(r^{\prime}\left|2 \pi m_{j}\right|^{\frac{1}{\beta}}\right)^{\alpha_{j}}}{\alpha_{j} !}\right)^{\beta} \\
& \leq \prod_{j} e^{\beta r^{\prime}\left|2 \pi m_{j}\right|^{\frac{1}{\beta}}} \leq e^{\beta \pi r^{\prime} d|m|^{\frac{1}{\beta}}}
\end{aligned}
$$

Remark: This implies that the functions which are analytic on an $r$-neighbourhood of the torus or the double torus are Gevrey $\beta$ with parameter $r$ for all $\beta>1$;

Sublemma 3.3 Let $f \in C_{r}^{G, \beta}\left(2 \mathbb{T}^{d}, g l(n, \mathbb{C})\right)$. Then for all $m \in \frac{1}{2} \mathbb{Z}^{d}$,

$$
\|\hat{f}(m)\| \leq\|f\|_{\beta, r}\left(1-\frac{1}{2^{\frac{\beta}{\beta-1}}}\right)^{1-\beta} e^{-\sum_{j}\left(2 \pi\left|m_{j}\right|\right)^{\frac{1}{\beta}} r}
$$


Proof: By definition of $\|f\|_{\beta, r}$,

$$
\sum_{\alpha \in \mathbb{N}^{d}}\left\|\widehat{\partial^{\alpha} f}(m)\right\| \frac{r^{\beta|\alpha|}}{\alpha !^{\beta}} \leq \sum_{\alpha \in \mathbb{N}^{d}} \sup _{\theta}\left\|\partial^{\alpha} f(\theta)\right\| \frac{r^{\beta|\alpha|}}{\alpha !^{\beta}}=\|f\|_{\beta, r}
$$

Now

$$
\partial^{\alpha} f(\theta) \sim \sum_{m} \hat{f}(m) \partial^{\alpha}\left(e^{2 i \pi\langle m, \theta\rangle}\right) \sim \sum_{m} \hat{f}(m) \prod_{j}\left(2 i \pi m_{j}\right)^{\alpha_{j}} \cdot\left(e^{2 i \pi\langle m, \theta\rangle}\right)
$$

thus

$$
\widehat{\partial^{\alpha} f}(m)=\prod_{j}\left(2 i \pi m_{j}\right)^{\alpha_{j}} \hat{f}(m)
$$

and therefore

$$
\|\hat{f}(m)\| \sum_{\alpha \in \mathbb{N}^{d}} \prod_{j}\left(2 \pi\left|m_{j}\right|\right)^{\alpha_{j}} \frac{r^{\beta \alpha_{j}}}{\alpha_{j} !^{\beta}} \leq\|f\|_{\beta, r}
$$

that is to say,

$$
\|\hat{f}(m)\| \prod_{j} \sum_{\alpha_{j} \in \mathbb{N}}\left(2 \pi\left|m_{j}\right|\right)^{\alpha_{j}} \frac{r^{\beta\left|\alpha_{j}\right|}}{\alpha_{j} !^{\beta}} \leq\|f\|_{\beta, r}
$$

Now, by Lemma 4.3 ,

$$
\sum_{\alpha_{j} \in \mathbb{N}}\left(2 \pi\left|m_{j}\right| r^{\beta}\right)^{\alpha_{j}} \frac{1}{\alpha_{j} !^{\beta}} \geq\left(1-\frac{1}{2^{\frac{\beta}{\beta-1}}}\right)^{\beta-1} e^{\left(2 \pi\left|m_{j}\right|\right)^{\frac{1}{\beta}} r}
$$

therefore

$$
\|\hat{f}(m)\| \leq\|f\|_{\beta, r}\left(1-\frac{1}{2^{\frac{\beta}{\beta-1}}}\right)^{1-\beta} e^{-\sum_{j}\left(2 \pi\left|m_{j}\right|\right)^{\frac{1}{\beta}} r}
$$

Lemma 3.4 Let $0<r \leq 1, f \in C_{r}^{G, \beta}\left(2 \mathbb{T}^{d}, g l(n, \mathbb{C})\right), N \in \mathbb{N}$ and $f^{N}$ the truncation of $f$ at $\operatorname{order} N$. Then for all $r^{\prime}<r$,

$$
\left\|f-f^{N}\right\|_{\beta, r^{\prime}} \leq C_{d, \beta}\|f\|_{\beta, r} N^{d+1} \frac{1}{\left(r-r^{\prime}\right)^{2(d+1)}} e^{-2\left(r-r^{\prime}\right) N^{\frac{1}{\beta}}}
$$

where $C_{d, \beta}$ only depends on $d, \beta$.

Proof: By definition,

$$
\left\|f-f^{N}\right\|_{\beta, r^{\prime}}=\sum_{\alpha} \sup _{\theta} \frac{r^{\prime \beta|\alpha|}}{\alpha !^{\beta}}\left|\partial^{\alpha}\left(f-f^{N}\right)(\theta)\right|
$$

Now 


$$
\left|\partial^{\alpha}\left(f-f^{N}\right)(\theta)\right| \leq \sum_{|m|>N}\|\hat{f}(m)\| \prod_{j=1}^{d}\left|m_{j}\right|^{\alpha_{j}}
$$

so by Sublemma 3.3,

$$
\left|\partial^{\alpha}\left(f-f^{N}\right)(\theta)\right| \leq\left(1-\frac{1}{2^{\frac{\beta}{\beta-1}}}\right)^{1-\beta}\|f\|_{\beta, r} \sum_{|m|>N} e^{-\sum_{l}\left(2 \pi\left|m_{l}\right|\right)^{\frac{1}{\beta}}} \prod_{j=1}^{d}\left|m_{j}\right|^{\alpha_{j}}
$$

whence

$$
\begin{aligned}
\left\|f-f^{N}\right\|_{\beta, r^{\prime}} & \leq\left(1-\frac{1}{2^{\frac{\beta}{\beta-1}}}\right)^{1-\beta}\|f\|_{\beta, r} \sum_{\alpha} \frac{r^{\prime \beta|\alpha|}}{\alpha !^{\beta}} \sum_{|m|>N} e^{-\sum_{l}\left(2 \pi\left|m_{l}\right|\right)^{\frac{1}{\beta}} r} \prod_{j=1}^{d}\left|m_{j}\right|^{\alpha_{j}} \\
& \leq\left(1-\frac{1}{2^{\frac{\beta}{\beta-1}}}\right)^{1-\beta}\|f\|_{\beta, r} \sum_{|m|>N} e^{-\sum_{l}\left(2 \pi\left|m_{l}\right|\right)^{\frac{1}{\beta}} r} \prod_{j=1}^{d} \sum_{\alpha_{j}} \frac{r^{\prime \beta \alpha_{j}}}{\alpha_{j} !^{\beta}}\left|m_{j}\right|^{\alpha_{j}}
\end{aligned}
$$

thus, using Lemma 4.3 ,

$$
\begin{aligned}
\left\|f-f^{N}\right\|_{\beta, r^{\prime}} & \leq\left(1-\frac{1}{2^{\frac{\beta}{\beta-1}}}\right)^{1-\beta}\|f\|_{\beta, r} \sum_{|m|>N} e^{-2\left(r-r^{\prime}\right) \sum_{j}\left|m_{j}\right|^{\frac{1}{\beta}}} \\
& \leq\left(1-\frac{1}{2^{\frac{\beta}{\beta-1}}}\right)^{1-\beta}\|f\|_{\beta, r} \sum_{|m|>N} e^{-2\left(r-r^{\prime}\right)|m|^{\frac{1}{\beta}}}
\end{aligned}
$$

and finally

$\left\|f-f^{N}\right\|_{\beta, r^{\prime}} \leq C_{d, \beta}\|f\|_{\beta, r} \sum_{M>N} M^{d} e^{-2\left(r-r^{\prime}\right) M^{\frac{1}{\beta}}} \leq C_{d, \beta}^{\prime}\|f\|_{\beta, r} \frac{N^{d+1}}{\left(r-r^{\prime}\right)^{2(d+1)}} e^{-2\left(r-r^{\prime}\right) N^{\frac{1}{\beta}}}$ where $C_{d, \beta}, C_{d, \beta}^{\prime}$ only depend on $d, \beta$.

\subsection{Reduction of the eigenvalues}

The reduction of the eigenvalues of a matrix $A$ at order $R, \bar{N}$ satisfies a good estimate in the Gevrey norm, as shows the following proposition:

Lemma 3.5 Let $R, N \in \mathbb{N} \backslash\{0\}, A \in \operatorname{gl}(n, \mathbb{C})$ and $\Phi$ a map of reduction of the eigenvalues of $A$ at order $R, \bar{N}$. Then $\Phi$ satisfies for all $r^{\prime}$ the Gevrey norm estimate

$$
\|\Phi\|_{\beta, r^{\prime}} \leq n C \cdot C_{0}\left(\frac{1+\left\|A_{\mathcal{N}}\right\|}{\kappa^{\prime \prime}}\right)^{n(n+1)} e^{2 \beta \pi r^{\prime} d \bar{N}^{\frac{1}{\beta}}}
$$

where $C$ only depends on $d$, and so does $\Phi^{-1}$. Moreover, if $\mathcal{G}=o(n)$ or $u(n)$, then 


$$
\|\Phi\|_{\beta, r^{\prime}} \leq n C e^{2 \beta \pi r^{\prime} d \bar{N}^{\frac{1}{\beta}}}
$$

and so does $\Phi^{-1}$.

Proof: For all $m \in \mathbb{Z}^{d}$ and all $r^{\prime}>0$, by Lemma 3.2,

$$
\left\|e^{2 i \pi\langle m,\rangle}\right\|_{\beta, r^{\prime}} \leq C e^{\beta \pi r^{\prime} d|m|^{\frac{1}{\beta}}}
$$

where $C$ only depends on $d$. Therefore

$$
\begin{aligned}
\|\Phi\|_{r^{\prime}} & \leq \sum_{L \in \mathcal{L}_{A, \kappa^{\prime \prime}}}\left\|P_{L}^{\mathcal{L}_{A, \kappa^{\prime \prime}}}\right\|\left\|e^{2 i \pi\left\langle m_{L}, \cdot\right\rangle}\right\|_{r^{\prime}} \\
& \leq C \sum_{L \in \mathcal{L}_{A, \kappa^{\prime \prime}}}\left\|P_{L}^{\mathcal{L}_{A, \kappa^{\prime \prime}}}\right\| e^{\beta \pi r^{\prime} d\left|m_{L}\right|^{\frac{1}{\beta}}} \\
& \leq C \sum_{L \in \mathcal{L}_{A, \kappa^{\prime \prime}}}\left\|P_{L}^{\mathcal{L}_{A, \kappa^{\prime \prime}}}\right\| e^{2 \beta \pi r^{\prime} d \bar{N}^{\frac{1}{\beta}}}
\end{aligned}
$$

Now by Lemma 2.1,

$$
\left\|P_{L}^{\mathcal{L}_{A, \kappa^{\prime \prime}}}\right\| \leq C_{0}\left(\frac{1+\left\|A_{\mathcal{N}}\right\|}{\kappa^{\prime \prime}}\right)^{n(n+1)}
$$

so (107) holds. If $\mathcal{G}$ is either $o(n)$ or $u(n)$, then $\mathcal{L}_{A, \kappa^{\prime \prime}}$ is a unitary decomposition and thus $P_{L}^{\mathcal{L}_{A, \kappa^{\prime \prime}}}$ has norm 1 , thus (108) holds.

\subsection{Homological equation}

Lemma 3.6 Let $0<r^{\prime}<r \leq 1, f \in C_{r}^{G, \beta}\left(2 \mathbb{T}^{d}, \mathcal{G}\right)$ and $g \in C_{r^{\prime}}^{G, \beta}\left(2 \mathbb{T}^{d}, \mathcal{G}\right)$. Let $C>0, D \geq 0$. Assume that for all $m \in \frac{1}{2} \mathbb{Z}^{d}$,

$$
\|\hat{g}(m)\| \leq C|m|^{D}|| \hat{f}(m) \|
$$

Then

$$
\|g\|_{\beta, r^{\prime}} \leq C^{\prime} C\|f\|_{\beta, r}\left(\frac{1}{r-r^{\prime}}\right)^{2 \beta(D+2) d}
$$

where $C^{\prime}$ only depends on $d, D, \beta$.

Proof: For all $\theta \in N \mathbb{T}^{d}$ and all $\alpha \in \mathbb{N}^{d}$,

$$
\begin{aligned}
\left\|\partial^{\alpha} g(\theta)\right\| & \leq \sum_{m \in \frac{1}{2} \mathbb{Z}^{d}}\|\hat{g}(m)\|\left|\partial^{\alpha} e^{2 i \pi\langle m, \theta\rangle}\right| \\
& \leq \sum_{m \in \frac{1}{2} \mathbb{Z}^{d}}\|\hat{g}(m)\| \prod_{j}\left|2 \pi m_{j}\right|^{\alpha_{j}}
\end{aligned}
$$


Therefore, by assumption,

$$
\begin{aligned}
\left\|\partial^{\alpha} g(\theta)\right\| & \leq C \sum_{m \in \frac{1}{2} \mathbb{Z}^{d}}|m|^{D}|| \hat{f}(m) \| \prod_{j}\left|2 \pi m_{j}\right|^{\alpha_{j}} \\
& \leq C^{\prime} C \sum_{m \neq 0} \frac{1}{|2 \pi m|^{2 d}} \prod_{j}\left|2 \pi m_{j}\right|^{\alpha_{j}+D+2}\|\hat{f}(m)\|
\end{aligned}
$$

so, letting $\overline{1}=(1, \ldots, 1)$,

$$
\begin{aligned}
& \left\|\partial^{\alpha} g(\theta)\right\| \leq C^{\prime} C \sum_{m \neq 0} \frac{1}{|2 \pi m|^{2 d}}\left\|\partial^{\alpha+(D+2)} \overline{1} f(m)\right\| \\
& \leq C^{\prime} C \sup _{\theta}\left\|\partial^{\alpha+(D+2) \overline{1}} f(\theta)\right\|
\end{aligned}
$$

where $C^{\prime}$ only depends on $d, D$, thus

$$
\begin{aligned}
\|g\|_{\beta, r^{\prime}} & =\sum_{\alpha \in \mathbb{N}^{d}}\left(r^{\prime}\right)^{\beta|\alpha|} \frac{1}{(\alpha !)^{\beta}} \sup _{\theta}\left\|\partial^{\alpha} g(\theta)\right\| \\
& \leq C^{\prime} C \sum_{\alpha}\left(r^{\prime}\right)^{\beta|\alpha|} \frac{1}{(\alpha !)^{\beta}} \sup _{\theta}\left\|\partial^{\alpha+(D+2) \overline{1}} f(\theta)\right\| \\
& \leq C^{\prime} C \sum_{\alpha} \frac{r^{\beta|\alpha+(D+2) \overline{1}|}}{((\alpha+(D+2) \overline{1}) !)^{\beta}} \sup _{\theta}\left\|\partial^{\alpha+(D+2) \overline{1}} f(\theta)\right\| \frac{r^{\prime \beta|\alpha|}}{r^{\beta|\alpha+(D+2) \overline{1}|}}\left(\frac{(\alpha+(D+2) \overline{1}) !}{\alpha !}\right)^{\beta} \\
& \leq C^{\prime} C\|f\|_{\beta, r} \sum_{\alpha} \frac{r^{\prime \beta|\alpha|}}{r^{\beta(|\alpha|+(D+2) d)}} \prod_{j}\left(\frac{\left(\alpha_{j}+D+2\right) !}{\alpha_{j} !}\right)^{\beta} \\
& \leq C^{\prime} C\|f\|_{\beta, r} \sum_{\alpha} \frac{r^{\prime \beta|\alpha|}}{r^{\beta(|\alpha|+(D+2) d)}}\left(\frac{(|\alpha|+D+2) !}{|\alpha| !}\right)^{\beta d} \\
& \leq C^{\prime} C\|f\|_{\beta, r} \sum_{\alpha} \frac{r^{\prime \beta|\alpha|}}{r^{\beta(|\alpha|+(D+2) d)}}(|\alpha|+D+2)^{\beta(D+2) d}
\end{aligned}
$$

Now the function

$$
\phi:\left[0,+\infty\left[\rightarrow \left[0,+\infty\left[, t \mapsto\left(\frac{r^{\prime}}{r}\right)^{t} t^{\beta(D+2) d}\right.\right.\right.\right.
$$

has its maximum at $t=\frac{\beta(D+2) d}{\ln \frac{r}{r^{\prime}}}$ where it takes the value $e^{-\beta(D+2) d}\left(\frac{\beta(D+2) d}{\ln \frac{r}{r^{\prime}}}\right)^{\beta(D+2) d}$. Therefore

$$
\begin{aligned}
\|g\|_{\beta, r^{\prime}} & \leq C^{\prime} C\|f\|_{\beta, r} e^{-\beta(D+2) d}\left(\frac{\beta(D+2) d}{r^{\prime} \ln \frac{r}{r^{\prime}}}\right)^{\beta(D+2) d} \\
& \leq C^{\prime} C\|f\|_{\beta, r} e^{-\beta(D+2) d} \frac{(\beta(D+2) d)^{\beta(D+2) d}}{\left(r-r^{\prime}\right)^{2 \beta(D+2) d}}
\end{aligned}
$$




\section{Proposition 3.7 Let}

- $N \in \mathbb{N}$,

- $\left.\left.\kappa^{\prime} \in\right] 0, \kappa\right]$,

- $\gamma \geq n(n+1)$,

- $0<r^{\prime}<r$.

Let $\tilde{A} \in \mathcal{G}$ with a $D C_{\omega}^{N}\left(\kappa^{\prime}, \tau\right)$ spectrum. Let $\tilde{F} \in C_{r}^{G, \beta}\left(2 \mathbb{T}^{d}, \mathcal{G}\right)$ with nice periodicity properties with respect to an $\left(\tilde{A}, \kappa^{\prime}, \gamma\right)$-decomposition $\mathcal{L}$. Then there exists a solution $\tilde{X} \in C_{r^{\prime}}^{G, \beta}\left(2 \mathbb{T}^{d}, \mathcal{G}\right)$ of equation

$$
\forall \theta \in 2 \mathbb{T}^{d}, \partial_{\omega} \tilde{X}(\theta)=[\tilde{A}, \tilde{X}(\theta)]+\tilde{F}^{N}(\theta)-\hat{\tilde{F}}(0) ; \quad \hat{\tilde{X}}(0)=0
$$

such that

- if $\tilde{F}$ has nice periodicity properties with respect to $\mathcal{L}$ et $\left(m_{L}\right)$, then so does $\tilde{X}$; in particular, if $\tilde{F}$ is defined on $\mathbb{T}^{d}$, then so is $\tilde{X}$,

- Let $\Phi$ be trivial with respect to $\mathcal{L}$ and a family $\left(m_{L}\right)$ such that for all $L,\left|m_{L}\right| \leq N^{\prime}$. There exists $C^{\prime}, D^{\prime}$ depending only on $n, d, \tau, \beta$ such that

$$
\left\|\Phi \tilde{X} \Phi^{-1}\right\|_{\beta, r^{\prime}} \leq C^{\prime}\left(\frac{\left(1+\left\|\tilde{A}_{\mathcal{N}}\right\|\right) N^{\prime}}{\left(r-r^{\prime}\right) \kappa^{\prime}}\right)^{D^{\prime} \gamma}\left\|\Phi \tilde{F} \Phi^{-1}\right\|_{\beta, r}
$$

Moreover, the truncation of $\tilde{X}$ at order $N$ is unique.

Proof: The existence of $\tilde{X}$, its unicity up to order $N$, the fact that it takes its values in $\mathcal{G}$ and its nice periodicity properties with respect to $\mathcal{L}$ are proved as in part 2 , proposition 2.8 .

To get the estimate (110), one first shows that for all $m \in \frac{1}{2} \mathbb{Z}^{d}$ and all $L, L^{\prime} \in \mathcal{L}^{\prime}$,

$$
\left\|P_{L}^{\mathcal{L}} \hat{\tilde{X}}(m) P_{L^{\prime}}^{\mathcal{L}}\right\| \leq C^{\prime} \frac{\left(1+\left\|\tilde{A}_{\mathcal{N}}\right\|\right)^{n^{2}-1}|m|^{\left(n^{2}-1\right) \tau}}{\kappa^{\prime\left(n^{2}-1\right)}}\left\|P_{L}^{\mathcal{L}} \hat{\tilde{F}}(m) P_{L^{\prime}}^{\mathcal{L}}\right\|\left(\left\|P_{L}^{\mathcal{L}}\right\|\left\|P_{L^{\prime}}^{\mathcal{L}}\right\|\right)^{n^{2}-1}
$$

It is done exactly as in proposition 2.8 to get (60). The estimate (111) and Lemma 3.6 imply

$$
\left\|P_{L}^{\mathcal{L}^{\prime}} \tilde{X} e^{2 i \pi\left\langle m_{L}-m_{L^{\prime}}\right\rangle} P_{L^{\prime}}^{\mathcal{L}^{\prime}}\right\|_{\beta, r^{\prime}} \leq C^{\prime \prime}\left(\frac{\left(1+\left\|\tilde{A}_{\mathcal{N}}\right\|\right) N^{\prime}}{\left(r-r^{\prime}\right) \kappa^{\prime}}\right)^{D \gamma}\left\|P_{L}^{\mathcal{L}^{\prime}} \tilde{F} e^{2 i \pi\left\langle m_{L}-m_{L^{\prime}}\right\rangle} P_{L^{\prime}}^{\mathcal{L}^{\prime}}\right\|_{\beta, r}
$$

where $C^{\prime \prime}, D$ only depend on $n, d, \tau, \beta$. Thus 
$\left\|\Phi \tilde{X} \Phi^{-1}\right\|_{\beta, r^{\prime}} \leq \sum_{L, L^{\prime}}\left\|P_{L}^{\mathcal{L}^{\prime}} \tilde{X} e^{2 i \pi\left\langle m_{L}-m_{L^{\prime}}\right\rangle} P_{L^{\prime}}^{\mathcal{L}^{\prime}}\right\|_{\beta, r} \leq C^{\prime \prime}\left(\frac{\left(1+\left\|\tilde{A}_{\mathcal{N}}\right\|\right) N^{\prime}}{\left(r-r^{\prime}\right) \kappa^{\prime}}\right)^{D \gamma} \sum_{L, L^{\prime}}\left\|P_{L}^{\mathcal{L}^{\prime}} \tilde{F} e^{2 i \pi\left\langle m_{L}-m_{L^{\prime}}\right\rangle} P_{L^{\prime}}^{\mathcal{L}^{\prime}}\right\|_{\beta, r}$

and therefore

$$
\left\|\Phi \tilde{X} \Phi^{-1}\right\|_{\beta, r^{\prime}} \leq C_{3}\left(\frac{(1+\|\tilde{A}\|) N^{\prime}}{\left(r-r^{\prime}\right) \kappa^{\prime}}\right)^{D^{\prime} \gamma}\left\|\Phi \tilde{F} \Phi^{-1}\right\|_{\beta, r}
$$

where $D^{\prime}, C_{3}$ only depend on $n, d, \tau, \beta$.

\subsection{Inductive lemmas}

In Gevrey regularity, we will need a Lemma which is almost identical to Lemma 2.9, apart from the presence of the parameter $\beta$, which is fixed and does not modify the proof:

Lemma 3.8 Let

- $\left.\kappa^{\prime} \in\right] 0,1[, C>0, \beta>1$;

- $\tilde{F} \in \mathcal{G}$,

- $\tilde{\epsilon}=\|\tilde{F}\|$,

- $\tilde{N} \in \mathbb{N}$,

- $\tilde{A} \in \mathcal{G}$ with $D C_{\omega}^{\tilde{N}}\left(\kappa^{\prime}, \tau\right)$ spectrum.

There exists a constant $c$ only depending on $n \tau, \beta$ such that if $\tilde{\epsilon}$ satisfies

$$
\tilde{\epsilon} \leq c\left(\frac{C^{\tau} \kappa^{\prime}}{1+\|\tilde{A}\|}\right)^{2 n}
$$

and

$$
\tilde{N} \leq \frac{|\log \tilde{\epsilon}|^{\beta}}{C}
$$

then $\tilde{A}+\tilde{F}$ has $D C_{\omega}^{\tilde{N}}\left(\frac{3 \kappa^{\prime}}{4}, \tau\right)$ spectrum.

Exactly as in the analytic case, one obtains the following inductive lemmas, where only the estimates slightly differ from their analytic analogues; since $\beta$ is fixed, the proofs go on in quite the same way:

\section{Proposition 3.9 Let}

- $\tilde{\epsilon}>0, \tilde{r} \leq 1, \tilde{r}^{\prime} \in\left[\frac{\tilde{r}}{2}, \tilde{r}\left[, \kappa^{\prime}>0, \tilde{N} \in \mathbb{N}, \gamma \geq n(n+1), C>0\right.\right.$;

- $\tilde{F} \in C_{\tilde{r}}^{G, \beta}\left(2 \mathbb{T}^{d}, \mathcal{G}\right), \tilde{A} \in \mathcal{G}$, 
- $\mathcal{L}$ an $\left(\tilde{A}, \kappa^{\prime}, \gamma\right)$-decomposition.

There exists a constant $C^{\prime \prime}>0$ depending only on $\tau, n, \beta$ such that if

1. $\tilde{A}$ has $D C_{\omega}^{\tilde{N}}\left(\kappa^{\prime}, \tau\right)$ spectrum;

2.

$$
\|\hat{\tilde{F}}(0)\| \leq \tilde{\epsilon} \leq C^{\prime \prime}\left(\frac{C^{\tau} \kappa^{\prime}}{1+\|\tilde{A}\|}\right)^{2 n}
$$

and

$$
\tilde{N} \leq \frac{|\log \tilde{\epsilon}|^{\beta}}{C}
$$

3. $\tilde{F}$ has nice periodicity properties with respect to $\mathcal{L}$

then there exist

- $C^{\prime} \in \mathbb{R}$ depending only on $n, d, \kappa, \tau, \beta$,

- $D \in \mathbb{N}$ depending only on $n, d, \tau, \beta$,

- $X \in C_{\tilde{r}^{\prime}}^{G, \beta}\left(2 \mathbb{T}^{d}, \mathcal{G}\right)$,

- $A^{\prime} \in \mathcal{G}$

- an $\left(A^{\prime}, \frac{3 \kappa^{\prime}}{4}, \gamma\right)$-decomposition $\mathcal{L}^{\prime}$

satisfying the following properties:

1. $A^{\prime}$ has $D C_{\omega}^{\tilde{N}}\left(\frac{3 \kappa^{\prime}}{4}, \tau\right)$ spectrum,

2. $\left\|A^{\prime}-\tilde{A}\right\| \leq \tilde{\epsilon}$;

3. the map $F^{\prime} \in C_{\tilde{r}^{\prime}}^{G, \beta}\left(2 \mathbb{T}^{d}, \mathcal{G}\right)$ defined by

$$
\forall \theta \in 2 \mathbb{T}^{d}, \partial_{\omega} e^{X(\theta)}=(\tilde{A}+\tilde{F}(\theta)) e^{X(\theta)}-e^{X(\theta)}\left(A^{\prime}+F^{\prime}(\theta)\right)
$$

has nice periodicity properties with respect to $\mathcal{L}^{\prime}$

4. If $\Phi$ is trivial with respect to $\mathcal{L}$ and a family $\left(m_{L}\right)$ satisfying, for all $L,\left|m_{L}\right| \leq N^{\prime}$, then

$$
\left\|\Phi^{-1} X \Phi\right\|_{\beta, \tilde{r}^{\prime}} \leq C^{\prime}\left(\frac{\left(1+\left\|\tilde{A}_{\mathcal{N}}\right\|\right) N^{\prime}}{\kappa^{\prime}\left(\tilde{r}-\tilde{r}^{\prime}\right)}\right)^{D \gamma}\left\|\Phi^{-1} \tilde{F} \Phi\right\|_{\beta, \tilde{r}}
$$


5. and if $\Phi$ is trivial with respect to $\mathcal{L}$ and a family $\left(m_{L}\right)$ satisfying, for all $L,\left|m_{L}\right| \leq$ $N^{\prime}$, then

$$
\begin{aligned}
\left\|\Phi^{-1} F^{\prime} \Phi\right\|_{\beta, \tilde{r}^{\prime}} & \leq C^{\prime}\left(\frac{\left(1+\left\|\tilde{A}_{\mathcal{N}}\right\|\right) N^{\prime}}{\kappa^{\prime}\left(\tilde{r}-\tilde{r}^{\prime}\right)}\right)^{D \gamma} e^{\left\|\Phi^{-1} X \Phi\right\|_{\beta, \tilde{r}^{\prime}}\left\|\Phi^{-1} \tilde{F} \Phi\right\|_{\beta, \tilde{r}}} \\
& \left(\|\Phi\|_{\beta, \tilde{r}}^{2}\left\|\Phi^{-1}\right\|_{\beta, \tilde{r}^{2}}^{2} \tilde{N}^{d} e^{-2 \pi \tilde{N}\left(\tilde{r}-\tilde{r}^{\prime}\right)}+\left\|\Phi^{-1} \tilde{F} \Phi\right\|_{\beta, \tilde{r}^{\prime}}\left(1+e^{\left.\left.\left\|\Phi^{-1} X \Phi\right\|_{\beta, \tilde{r}^{\prime}}\right)\right)}\right.\right.
\end{aligned}
$$

Moreover, if $\tilde{F}$ is continuous on $\mathbb{T}^{d}$, then so are $X$ and $F^{\prime}$. If $\mathcal{G}=o(n)$ or $u(n)$, then the same holds replacing condition (114) by

$$
\|\hat{\tilde{F}}(0)\| \leq \tilde{\epsilon} \leq C^{\prime \prime}\left(C^{\tau} \kappa^{\prime}\right)^{2}
$$

In Proposition [3.9, the only difference with Proposition 2.13 is the introduction of the parameters $\beta$ and $N^{\prime}$. The inductive step is formulated exactly as proposition 2.14 , with the only difference that the parameters $N, R$ will be chosen as

$$
\left\{\begin{array}{c}
N(r, \epsilon)=\left(\frac{1}{2 \pi r}|\log \epsilon|\right)^{\beta} \\
R\left(r, r^{\prime}\right)=\left[\frac{1}{\left(r-r^{\prime}\right)^{8}} 80^{4}\left(\frac{1}{2} n(n-1)+1\right)^{2}\right]^{\beta}
\end{array}\right.
$$

and not as in (79). Note that the parameter $N^{\prime}$ in the estimates of properties 4 and 5 in proposition 3.9 does not modify essentially the proof, once it is instantiated by $\bar{N}$. The statement and the proof of the main theorem are identical.

\section{Appendix}

\subsection{Spectrum of a one-parameter family of matrices}

Lemma 4.1 Let $\mathcal{G}$ be a Lie algebra and $A, F \in \mathcal{G}$ with $\|F\| \leq 1$. Let $\alpha_{1}(\lambda), \ldots, \alpha_{n}(\lambda)$ be a continuous choice of the eigenvalues of $A+\lambda F$ as $\lambda$ varies from 0 to 1 . Then for all $1 \leq j \leq n$, there exists $1 \leq j^{\prime} \leq n$ such that

$$
\left|\alpha_{j^{\prime}}(\lambda)-\alpha_{j}(0)\right| \leq 2 n \lambda^{\frac{1}{n}}(\| A||+1)
$$

Proof: Fix $j \leq n$. For every $\lambda$, let

$$
A(\lambda)=A+\lambda F
$$

and

$$
f(\lambda)=\operatorname{det}\left(\alpha_{j}(0) I-A(\lambda)\right)
$$

Then $f(0)=0$ and for every $\lambda$,

$$
f(\lambda)=\operatorname{det}\left(\alpha_{j}(0) I-A(\lambda)\right)=\prod_{j^{\prime}}\left(\alpha_{j}(0)-\alpha_{j^{\prime}}(\lambda)\right)
$$




$$
\left|\prod_{j^{\prime}}\left(\alpha_{j}(0)-\alpha_{j^{\prime}}(\lambda)\right)\right|=|f(0)-f(\lambda)| \leq \sup _{\lambda^{\prime \prime}}\left|f^{\prime}\left(\lambda^{\prime \prime}\right)\right||\lambda|
$$

and since

$$
\begin{aligned}
\left|f^{\prime}\left(\lambda^{\prime \prime}\right)\right| & =\left|\sum_{\sigma} \frac{d}{d \lambda^{\prime \prime}} \prod_{k}\left(\alpha_{j}(0) I-A-\lambda^{\prime \prime} F\right)_{k, \sigma(k)}\right| \\
& \leq n n !\left[\|A\|+\left\|A\left(\lambda^{\prime \prime}\right)\right\|\right]^{n-1} \\
& \leq 2^{n-1} n n ![\|A\|+1]^{n-1}
\end{aligned}
$$

then there exists $j^{\prime}$ such that

$$
\left|\alpha_{j}(0)-\alpha_{j^{\prime}}(\lambda)\right| \leq 2 n|\lambda|^{\frac{1}{n}}[|| A||+1]
$$

In case $G$ is compact, we have the following lemma (see [5], lemma A.5):

Lemma 4.2 Let $\mathcal{G}=o(n)$ or $u(n)$ and $A, F \in \mathcal{G}$ with $\|F\| \leq 1$. Let $\alpha_{1}(\lambda), \ldots, \alpha_{n}(\lambda)$ be an analytic choice of the eigenvalues of $A+\lambda F$ as $\lambda$ varies from 0 to 1 . Then for all $1 \leq j \leq n$,

$$
\left|\alpha_{j}(\lambda)-\alpha_{j}(0)\right| \leq \lambda
$$

Proof: For each $\lambda$, let $p_{1}(\lambda), \ldots, p_{n}(\lambda)$ be an orthonormal basis of eigenvectors of $A+\lambda F$ (take them analytic in $\lambda$ ). Then for each $1 \leq j \leq n$, one can assume

$$
(A+\lambda F) p_{j}(\lambda)=\alpha_{j}(\lambda) p_{j}(\lambda)
$$

and derivating this along $\lambda$, one gets

$$
\left(A+\lambda F-\alpha_{j}(\lambda)\right) p_{j}^{\prime}(\lambda)+\left(F-\alpha_{j}^{\prime}(\lambda)\right) p_{j}(\lambda)=0
$$

Now let $\beta_{1}, \ldots, \beta_{n}$ be such that

$$
p_{j}^{\prime}(\lambda)=\sum_{l=1}^{n} \beta_{l} p_{l}(\lambda)
$$

Then

$$
\sum_{l \neq j} \beta_{l}\left(A+\lambda F-\alpha_{j}(\lambda)\right) p_{l}(\lambda)+\left(F-\alpha_{j}^{\prime}(\lambda)\right) p_{j}(\lambda)=0
$$

and taking the scalar product with $p_{j}(\lambda)$,

$$
\left\langle\left(F p_{j}(\lambda), p_{j}(\lambda)\right\rangle=\alpha_{j}^{\prime}(\lambda)\right.
$$

Therefore

$$
\left|\alpha_{j}(0)-\alpha_{j}(\lambda)\right| \leq|\lambda| \sup _{\lambda^{\prime}}\left|\alpha_{j}^{\prime}\left(\lambda^{\prime}\right)\right| \leq \lambda \square
$$




\subsection{A lemma on integer series with non-negative terms}

The following lemma was proven by D. Sauzin and is used in Section 3 ,

Lemma 4.3 Let $a>0$. For all $r \geq 0$, consider $E_{a}(r)=\sum_{k \geq 0} \frac{r^{k}}{k^{! a}}$. Then

$$
\left\{\begin{array}{l}
K_{1} e^{\lambda a r \frac{1}{\alpha}} \leq E_{a}(r) \leq e^{a r^{\frac{1}{a}}} \quad \text { if } \quad a>1,0<\lambda<1 \\
e^{a r^{\frac{1}{a}}} \leq E_{a}(r) \leq K_{1} e^{\lambda a r^{\frac{1}{a}}} \quad \text { if } \quad 0<a<1, \lambda>1
\end{array}\right.
$$

where

$$
K_{1}=\left(1-\lambda^{\frac{a}{a-1}}\right)^{a-1}<1
$$

Proof: One uses the following inequalities: if $\alpha>1$ and $\left(X_{k}\right)_{k \in \mathbb{N}},\left(Y_{k}\right)_{k \in \mathbb{N}}$ are families of non-negative numbers,

$$
\sum X_{k}^{\alpha} \leq\left(\sum X_{k}\right)^{\alpha}
$$

and

$$
\sum X_{k}^{\alpha} \geq \frac{\left(\sum X_{k} Y_{k}\right)^{\alpha}}{\left(\sum Y_{k}^{\beta}\right)^{\frac{\alpha}{\beta}}}
$$

for $\beta=\frac{\alpha}{\alpha-1}$, if $\sum X_{k} Y_{k}<\infty$. Note that (122) is equivalent to

$$
\sum x_{k}^{\frac{1}{\alpha}} \geq\left(\sum x_{k}\right)^{\frac{1}{\alpha}}
$$

with $X_{k}=x_{k}^{\frac{1}{\alpha}}$. Also (123) is equivalent to

$$
\sum x_{k}^{\frac{1}{\alpha}} \leq\left(\sum y_{k}^{\frac{\alpha}{\alpha-1}}\right)^{1-\frac{1}{\alpha}}\left(\sum \frac{x_{k}}{y_{k}}\right)^{\frac{1}{\alpha}}
$$

with $X_{k}=\left(\frac{x_{k}}{y_{k}}\right)^{\frac{1}{\alpha}}$ and $y_{k}=Y_{k}^{\alpha}$.

1. In the first case, apply (122) and (123) with $\alpha=a, X_{k}=\frac{r^{\frac{k}{\alpha}}}{k !}$ and $Y_{k}=\lambda^{k}$.

2. In the second case, apply (124) and (125) with $\alpha=\frac{1}{a}, x_{k}=\frac{r^{\frac{k}{a}}}{k !}$ and $y_{k}=\lambda^{-k}$.

\section{Acknowledgments}

The author would like to thank Håkan Eliasson for his supervision, as well as David Sauzin, Raphaël Krikorian, Artur Avila and Xuanji Hou for suggestions and useful discussions. 


\section{References}

[1] Avila, A., Krikorian, R.: Reducibility or non-uniform hyperbolicity for quasi-periodic Schrödinger cocycles, Ann. of Math. 164, 911-940 (2006)

[2] Chavaudret, C.: Reducibility of quasi-periodic cocycles in linear Lie groups, arxiv:0810 0651, accepted for publication in Ergod. Theory and Dyn. Syst.

[3] Eliasson, L.H.: Almost reducibility of linear quasi-periodic systems, Proc. Sympos. Pure Math. 69, 679-705 (2001)

[4] Eliasson, L.H.: Floquet solutions for the 1-dimensional quasi-periodic Schrödinger equation, Comm. Math. Phys. 146, 447-482 (1992)

[5] Eliasson, L.H.: Discrete one-dimensional quasi-periodic Schrödinger operators with pure point spectrum, Acta Math. 179, 153-196 (1997)

[6] Hadj Amor, S.: Opérateur de Schrödinger quasi-périodique unidimensionnel, Thèse de Doctorat de l'Université Paris 7, 2006

[7] Krikorian, R.: Réductibilité des systèmes produit croisé à valeurs dans des groupes compacts, Astérisque 259 (1999)

[8] J.P.Marco, D.Sauzin, Stability and instability for Gevrey quasi-convex near-integrable hamiltonian systems, Publications mathématiques de l'IHES, 96 (2003), 199-275 